\title{
MODULAR REPRESENTATION THEORY OF FINITE GROUPS WITH T. I. SYLOW $p$-SUBGROUPS
}

\author{
H. I. BLAU AND G. O. MICHLER
}

\begin{abstract}
Let $p$ be a fixed prime, and let $G$ be a finite group with a T.I. Sylow $p$-subgroup $P$. Let $N=N_{G}(P)$ and let $k(G)$ be the number of conjugacy classes of $G$. If $z(G)$ denotes the number of $p$-blocks of defect zero, then we show in this article that $z(G)=k(G)-k(N)$. This result confirms a conjecture of J. L. Alperin. Its proof depends on the classification of the finite simple groups. Brauer's height zero conjecture and the Alperin-McKay conjecture are also verified for finite groups with a T.I. Sylow $p$-subgroup.
\end{abstract}

\section{INTRODUCTION}

Let $p$ be a fixed prime, and let $G$ be a finite group with a T.I. Sylow $p$ subgroup $P$. That is, two different conjugates of $P$ have only the identity element in common. At the Santa Cruz conference on finite groups J. L. Alperin [1] stated the following conjecture: If $P$ is T.I. then the number of ordinary irreducible characters of $G$ not vanishing on the nonidentity elements of $P$ equals the number of ordinary irreducible characters of the normalizer $N_{G}(P)$ of $P$. It is the purpose of this article to show that Alperin's conjecture follows from the classification theorem of the finite simple groups.

In fact we prove the block version of Alperin's conjecture. In order to state it we need the following notation. For any $p$-block $B$ with defect group $\delta(B)={ }_{G} D$ of a finite group $G$ the numbers of ordinary irreducible characters, of modular irreducible characters, and of ordinary irreducible characters $\chi$ of $B$ with height $\operatorname{ht}(\chi)=0$ are denoted by $k(B), l(B)$ and $k_{0}(B)$, respectively. Let $z(G)$ be the number of $p$-blocks of $G$ with defect zero. The numbers of all ordinary irreducible characters and of all modular irreducible characters of $G$ are denoted by $k(G)$ and $l(G)$, respectively. Throughout this article $(F, R, S)$ denotes a splitting $p$-modular system for the finite group $G$, where $R$ is a complete discrete rank one valuation ring of characteristic zero with maximal ideal $\pi R \geq p R$, and where $F=R / \pi R$ and $S=q u o t(R)$ are splitting fields for $G$ and all its subgroups.

Received by the editors August 31, 1988.

1980 Mathematics Subject Classification (1985 Revision). Primary $20 \mathrm{C} 20$.

Key words and phrases. Blocks of defect zero, characters of height zero, modular characters, conjugacy classes. 
Let $G$ be a finite group with a T.I. Sylow $p$-subgroup $P$. Let $B$ be a $p$-block with defect group $\delta(B)={ }_{G} P$ and Brauer correspondent $b$ in $N=N_{G}(P)$. Then our main theorem (9.2) asserts that $k(B)=k(b), k_{0}(B)=k_{0}(b)$ and $l(B)=l(b)$. In particular, $k(G)=k(N)+z(G)$.

As an application we obtain in Corollary 9.3 that Brauer's height zero conjecture holds for groups with T.I. Sylow subgroups. Hence $k(B)=k_{0}(B)$ if and only if $P$ is abelian. If $P$ is not normal in $G$, then $k(B) \leq|P|$ and $k_{0}(B) \leq\left|P / P^{\prime}\right|$ by Corollary 9.4 .

Since the blocks $B$ and $b$ are stably equivalent $F$-algebras, Theorem 9.2 supports the long standing conjecture in the theory of finite dimensional algebras asserting that two indecomposable stably equivalent algebras $B$ and $b$ have the same number $l(B)=l(b)$ of nonisomorphic nonprojective simple modules (Remark 9.5).

In $\S 1$ the group structure of a finite group $G$ with a T.I. Sylow $p$-subgroup $P$ is described. Here we quote mainly results of Puig [19] and Suzuki [24]. Furthermore, the classification theorem for all finite simple groups $G$ with a noncyclic T.I. Sylow $p$-subgroup is restated. Using Fong's reduction theorems we show in $\S 3$ that it suffices to prove the main theorem for those groups $X$ which are cyclic central extensions of automorphism groups of such a simple group $G$.

In $\S 2$ methods are presented for counting the block invariants of a block $B$ and its Brauer correspondent in overgroups $A$ of finite groups $G$ such that $A / G$ is cyclic. They reduce the problem to counting the fixed points of automorphisms on the sets of irreducible characters. In order to find them we determine the conjugacy classes of $\mathrm{SL}_{2}(q), \mathrm{SU}_{3}\left(q^{2}\right),{ }^{2} B_{2}(q),{ }^{2} G_{2}(q)$ and certain overgroups of $\mathrm{SL}_{2}(q)$ and $\mathrm{SU}_{3}\left(q^{2}\right)$ fixed by automorphisms in $\S 4$. These subsidiary results are used in $\S \S 5$ and 6 to determine the block invariants of the relevant extensions of the finite groups of Lie type having a T.I. Sylow $p$ subgroup. Since the Schur multiplier of $\operatorname{PSL}_{3}(4)$ is rather large, we study this case separately in $\S 7$. The block invariants of the groups involving the simple sporadic groups with T.I. Sylow $p$-subgroups are computed in $\S 8$. The final section (§9) contains the proof of the main theorem and its above-mentioned corollaries.

For notation and terminology we refer to the books by Aschbacher [2], Carter [6], Feit [11], Huppert [14] and to the Atlas [8].

\section{Structure of groups with T.I. Sylow $p$-subgroups}

Let $X$ be a finite group with a T.I. Sylow $p$-subgroup $P \neq 1$. Then $P \cap P^{X}=$ 1 for all $x \in X$ with $x \notin N_{X}(P)$. Suzuki determined the structure of a group $X$ with a T.I. Sylow 2-subgroup $P$ in his paper [24]. For odd primes $p$ the following subsidiary lemma and propositions are special cases of results of Gorenstein-Lyons [12] and Puig [19]. They are restated here for the convenience of the reader. 
Lemma 1.1. Let $X$ be a finite group with a nonnormal T.I. Sylow p-subgroup $P$. Then the following assertions hold:

(a) If $X$ is p-solvable, then $P$ is either cyclic or a generalized quaternion 2-group.

(b) If $P$ is a generalized quaternion 2-group, then either $X=O(X) P$ or $X / O(X) \simeq \mathrm{SL}_{2}(3)$.

Proof. See Puig [19, p. 27] and Suzuki [24, pp. 58 and 63].

Proposition 1.2. Let $X$ be a finite group with a nonnormal T.I. Sylow p-subgroup $P$. Suppose that $P$ is neither cyclic nor a generalized quaternion 2-group. Let $H=O^{p^{\prime}}(X)$ and $U=O^{p}(H)$. Then the following assertions hold:

(a) $O_{p^{\prime}}(X) \leq N_{X}(P)$.

(b) $O_{p^{\prime}}(H)<U \leq U P=H$.

(c) $X=U N_{X}(P)$.

(d) $G=U / O_{p^{\prime}}(H)$ is a nonabelian simple group with a T.I. Sylow $p$ subgroup.

(e) $X / O_{p^{\prime}}(X)$ acts faithfully on $G$ via conjugation.

(f) $O_{p^{\prime}}(X)=C_{X}(H)$.

(g) $O_{p^{\prime}}(H)=Z(H)$.

(h) $H=U$ unless $p=3$ and $G \simeq \operatorname{PSL}(2,8)$ or $p=5$ and $G \simeq{ }^{2} B_{2}\left(2^{5}\right)$.

(i) $Z(U)=Z(H)$ is a factor group of the Schur multiplier of $G$.

Proof. Assertions (a)-(g) are immediate consequences of Propositions 3-7 of Puig [19, pp. 26-29].

From Theorems (24.1) and (24.9) of Gorenstein-Lyons [12, pp. 307 and 318], respectively, it follows that $H \neq U=O^{p}(H)$ if and only if $H / O_{p^{\prime}}(H) \in$ $\left\{{ }^{2} G_{2}(3)\right.$, Aut $\left.{ }^{2} B_{2}\left(2^{5}\right)\right\}$ and $p \in\{3,5\}$, respectively. For details, see Proposition 2.2 of [3].

By Proposition 5 of Puig [19, p. 28], $U$ is the unique minimal normal subgroup $N$ of $X$ with $p \| N \mid$. Since $G \simeq U / O_{p^{\prime}}(U)$ is simple and nonabelian, assertion (g) implies that $U$ is a perfect central extension of $G$. Thus (i) holds.

Proposition 1.3. Let $G$ be a nonabelian simple group with a noncyclic T.I. Sylow p-subgroup $P$. Then $G$ is ismomorphic to one of the following groups:

(a) $\operatorname{PSL}_{2}(q)$, where $q=p^{n}$ and $n \geq 2$.

(b) $\operatorname{PSU}_{3}\left(q^{2}\right)$, where $q=p^{n}$.

(c) $p=2$ and $G \simeq{ }^{2} B_{2}\left(2^{2 m+1}\right)$.

(d) $p=3$ and $G \simeq{ }^{2} G_{2}\left(3^{2 m+1}\right)$ and $m \geq 1$.

(e) $p=3$ and $G \simeq \operatorname{PSL}_{3}$ (4) or $M_{11}$.

(f) $p=5$ and $G \simeq{ }^{2} F_{4}(2)^{\prime}$ or $M^{c} L$.

(g) $p=11$ and $G \simeq J_{4}$.

Proof. This result is a restatement of Theorem 1 of Suzuki $[24$, p. 58] in the case where $p=2$. For odd primes $p$ it follows from the classification theorem of finite simple groups and Theorem (24.1) of Gorenstein-Lyons [12, p. 307]. 


\section{Counting Characters}

In this section we state some subsidiary results for the computation of the numbers of the ordinary irreducible characters of a $p$-block $B$ of finite group $G$ and of its Brauer correspondent $b$ in $N=N_{G}(P)$, respectively.

The following well-known consequence of Brauer's second main theorem will be used frequently.

Lemma 2.1. Let $G$ be a finite group with a T.I. Sylow p-subgroup P. Let $B$ be a p-block of $G$ with defect group $\delta(B)={ }_{G} P$, and let $b$ be its Brauer correspondent in $N=N_{G}(P)$. Then $k(B)=k(b)$ if and only if $l(B)=l(b)$.

Proof. Since $P$ is a T.I. Sylow $p$-subgroup of $G$ it is easy to see that $C_{G}(y) \leq N$ for every $1 \neq y \in P$. Therefore $k(B)-l(B)=k(b)-l(b)$ by Lemma 6.6(ii) and (iv) of Feit [11, p. 175], which implies the assertion.

Lemma 2.2. Let a finite group $A$ act as a permutation group on each of the two finite sets $S_{1}$ and $S_{2}$. Let $H$ be a subgroup of $A$, and let $f_{i}(H)$ be the number of fixed points of $H$ in $S_{i}$. Let $m_{i}(H)$ be the number of points $s$ of $S_{i}$ having $H$ as their stabilizer $C_{A}(s)=\left\{a \in A \mid s^{a}=s\right\}$ in $A$ for $i=1,2$.

If $f_{1}(H)=f_{2}(H)$ for all subgroups $H \leq A$, then $m_{1}(H)=m_{2}(H)$ for all subgroups $H$ of $A$.

Proof. Clearly $m_{i}(H) \leq f_{i}(H)$ for all subgroups $H \leq A$, and $f_{i}(A)=m_{i}(A)$ for $i=1,2$. Thus $m_{1}(A)=m_{2}(A)$.

To prove the assertion we proceed by induction on $|A: H|$. Now $m_{i}(H)=$ $f_{i}(H)-\sum_{A \geq J>H} m_{i}(J)$. Let $H<A$ be a proper subgroup of $A$. Then $m_{1}(H)=$ $f_{1}(H)-\left[\sum_{A \geq J>H} m_{1}(J)\right]$. Thus by induction

$$
m_{1}(H)=f_{2}(H)-\left[\sum_{A>J>H} m_{2}(J)\right]-m_{2}(A)=m_{2}(H) .
$$

This completes the proof.

Proposition 2.3. Suppose $X$ is a finite group, $H \leq G \triangleleft X$ with $X / G$ cyclic. Suppose that $y \in X$ generates $X / G$ and normalizes $H$ and that $|X: G|=$ $|H\langle y\rangle: H|$. Let $B$ be a union of $p$-blocks of $G$ and $b$ a union of p-blocks of $H$ for some prime $p$ such that $y$ stabilizes $B$ and $b$. Let $S_{i}(B)$ denote either the set of irreducible characters in $B$ of height zero, the set of all irreducible characters in $B$ or the set of irreducible Brauer characters in $B$, as $i=0,1,2$ respectively. Define $S_{i}(b)$ similarly. Suppose that, for some $i$, for all subgroups $C$ of $\langle y\rangle$, the number $f\left(S_{i}(B), C\right)$ of points of $S_{i}(B)$ fixed by $C$ equals the corresponding number $f\left(S_{i}(b), C\right)$. Let $B_{1}$ be the union of all p-blocks of $X$ which cover $B$, and $b_{1}$ the union of all $p$-blocks of $H\langle y\rangle$ which cover $b$.

(a) If $i=1$ or 2 , then $\left|S_{i}\left(B_{1}\right)\right|=\left|S_{i}\left(b_{1}\right)\right|$.

(b) If $i=0$ and $p \nmid|X / G|$, then $\left|S_{0}\left(B_{1}\right)\right|=\left|S_{0}\left(b_{1}\right)\right|$.

Proof. (a) Let $Y=H\langle y\rangle$. Let $\mathscr{C}$ equal the set of subgroups $C$ of $\langle y\rangle$ which contain $\langle y\rangle \cap H$. Then $\mathscr{C}$ is in bijection with the set of subgroups of $X$ which 
contain $G$ and with the set of subgroups of $Y$ which contain $H$, via the correspondences $C G \leftrightarrow C \leftrightarrow C H$.

For every $\psi \in S_{i}\left(B_{1}\right)$ there is a constituent $\chi$ of $\psi_{\mid G}$ belonging to $S_{i}(B)$ such that $\psi$ is a constituent of $\chi^{X}$. As $X / G$ is cyclic, Clifford's theorem and Theorem 2.14 of [11, p. 102] imply that $\psi_{\mid G}=\sum_{s=1}^{e(\chi)} \chi_{s}$, where $T=T_{X}(\chi)$ is the inertial subgroup of $\chi$ in $X$, and where $\chi_{1}=\chi, \chi_{2}, \ldots, \chi_{e(x)}$ belong to an $X / T$-orbit $O(\chi)$ of $e(\chi)=|X: T|$ inequivalent characters of $S_{i}(B)$ with inertial subgroup $T$. Furthermore, $|T / G|$ many characters in $S_{i}\left(B_{1}\right)$ cover a given $X / T$-orbit.

For each $C \in \mathscr{C}$ let $n_{1}(C)$ and $n_{2}(C)$ be the numbers of $X / C G$-orbits and $Y / C H$-orbits of characters $\chi \in S_{i}(B)$ and $\mu \in S_{i}(b)$ with inertial subgroups $T_{X}(\chi)=C G$ and $T_{Y}(\mu)=C H$, respectively. Then by the first two paragraphs of the proof we have

$$
\begin{aligned}
\left|S_{i}\left(B_{1}\right)\right| & =\sum_{C \in \mathscr{C}} n_{1}(C)|C G: G|, \\
\left|S_{i}\left(b_{1}\right)\right| & =\sum_{C \in \mathscr{C}} n_{2}(C)|C H: H| .
\end{aligned}
$$

By hypothesis, $f\left(S_{i}(B), C\right)=f\left(S_{i}(b), C\right)$ for all subgroups $C$ of $\langle y\rangle$. By Lemma 2.2, the number $m_{1}(C)$ of characters $\chi \in S_{i}(B)$ with inertial subgroup $C G$ equals the number $m_{2}(C)$ of characters $\mu \in S_{i}(b)$ with inertial subgroup $\mathrm{CH}$. Hence

$$
n_{1}(C)|X: C G|=m_{1}(C)=m_{2}(C)=n_{2}(C)|Y: C H| .
$$

Since $|X: C G|=|Y: C H|$, we have $n_{1}(C)=n_{2}(C)$ for all $C \in \mathscr{C}$. As $|C G: G|=|C H: H|,(*)$ and $(* *)$ imply that $\left|S_{i}\left(B_{i}\right)\right|=\left|S_{i}\left(b_{i}\right)\right|$ for $i=1,2$.

(b) As $p \nmid|X: T|=|Y: H|$, a block of $G$ and the blocks of $X$ covering it have the same defect. So each constituent $\chi$ of $\psi_{\mid G}$ for $\psi \in S_{1}\left(B_{1}\right)$ has height zero if and only if $\psi$ has height zero. Therefore the above argument yields $\left|S_{0}\left(B_{1}\right)\right|=\left|S_{0}\left(b_{1}\right)\right|$. This completes the proof.

\section{REDUCTION TO ALMOST SIMPLE GROUPS}

The following result contains most of the main theorem of this paper. Its proof comprises the next five sections, and is not completed until Lemma 9.1 is established.

Proposition 3.1. Let $X$ be a finite group with a T.I. Sylow p-subgroup P. Let $B$ be a p-block of $X$ with defect group $\delta(B)={ }_{X} P$ and Brauer correspondent $b$ in $N=N_{X}(P)$. Then the following assertions hold:

(a) $k(B)=k(b)$.

(b) $k_{0}(B)=k_{0}(b)$. 
Let $X$ be a finite group and $H=O^{p^{\prime}}(X)$. If $Z(X)=O_{p^{\prime}}(X)=Z(H)=$ $C_{X}(H)$ is cyclic and $G=H / Z(H)$ is a nonabelian simple group, then $X$ is called almost simple (w.r.t. $p$ ).

We show in this section that if $X$ is a counterexample to Proposition 3.1, then $P$ is neither cyclic nor quaternion and $X$ may be assumed almost simple. Hence $G:=H / Z(H)$ is one of the groups listed in Proposition 1.3. The various possibilities for $G$ are treated in $\S \S 4-8$.

All results in this section will be applied to groups which satisfy the hypotheses of Proposition 3.1. Since the assumptions of our lemmas are not always the same, and since we wish to keep our results unambiguous, we state all hypotheses explicitly with each assertion.

Lemma 3.2. Let $X$ be a finite group with a T.I. Sylow p-subgroup P. Let $B$ be a p-block of $X$ with defect group $\delta(B)={ }_{X} P$ and Brauer correspondent $b$ in $N=N_{X}(P)$.

(a) If $P$ is cyclic, then $k(B)=k_{0}(B)=k_{0}(b)=k(b) \leq|P|$.

(b) If $P$ is a generalized quaternion 2-group, then either $|P|=2^{m+1}, l(B)=$ $l(b)=1$ and $k(B)=k(b)=2^{m-1}+3$; or $|P|=8, l(B)=l,(b)=3$ and $k(B)=k(b)=7$. In any case, $k_{0}(B)=k_{0}(b)=4=\left|P / P^{\prime}\right|$.

Proof. Assertion (a) is an immediate consequence of Dade's theorem (see [11, pp. 277 and 278]).

(b) By Lemma 1.1 either $X=O(X) P$ or $X / O(X) \simeq \mathrm{SL}_{2}(3)$. In the first case $X$ and $N$ are 2-nilpotent groups. Hence $l(B)=l(b)=1$, and the block ideals $B$ and $b$ are isomorphic to full rings of matrices over the group algebra $F P$ by Theorems $C$ and $H$ of Külshammer [16] and Satz 25.3 of Huppert [14]. Thus $k(B)=k(b)=2^{m-1}+3$ and $k_{0}(B)=k_{0}(b)=4=\left|P / P^{\prime}\right|$.

In the second case the block ideals $B$ and $b$ have the same block invariants as the group algebra $\mathrm{SL}_{2}(3)$ by Theorems $\mathrm{C}$ and $\mathrm{H}$ of Külshammer [16] and Satz 25.5 of Huppert [14]. Thus $k(B)=k(b)=7$ and $k_{0}(B)=k_{0}(b)=4=\left|P / P^{\prime}\right|$.

For every ring $A$ and every natural number $m$ the ring of all $m \times m$ matrices over $A$ is denoted by $A_{m}$. If $\gamma \in Z^{2}\left(Y, F^{*}\right)$ is a 2-cocycle of the group $Y$, then $F_{\gamma} Y$ denotes the twisted group algebra of $Y$ over the field $F$ with respect to the 2-cocycle $\gamma$.

The next result shows that in order to establish Proposition 3.1 for a group $X$, it suffices to assume that $O_{p^{\prime}}(X)=Z(X)$ and $Z(X)$ is cyclic.

Lemma 3.3. Let $X$ be a finite group with a nonnormal T.I. Sylow p-subgroup $P$. Suppose that $P$ is neither cyclic nor a generalized quaternion 2-group. Let $H=O^{p^{\prime}}(X), U=O^{p}(H), K=O_{p^{\prime}}(X)$ and $L=K U$. Let $B$ be a p-block of $X$ with defect group $\delta(B)={ }_{X} P$ and Brauer correspondent $b$ in $N=N_{X}(P)$. Then the following assertions hold:

(a) $G=U / O_{p^{\prime}}(U) \simeq L / O_{p^{\prime}}(L)$ is a nonabelian simple group with T.I. Sylow p-subgroup $P_{1} \simeq P \cap L$. 
(b) $B$ and $b$ cover a p-block $f$ of $K$ with inertial subgroup $T=T_{X}(f) \geq P$.

(c) $H \leq T$. Let $B_{1}$ and $b_{1}$ be the Fong correspondents of $B$ and $b$ in $T$ and $N_{T}=N_{T}(P)$, respectively. Then $b_{1}$ is the Brauer correspondent of $B_{1}$, and the Fong correspondences preserve block invariants.

For the remaining assertions assume that $X=T$, so that $B_{1}=B$ and $b_{1}=b$. Let $\bar{X}=X / K, \bar{P}=P K / K$ and $\bar{N}=N_{\bar{X}}(\bar{P})$.

(d) There exists a 2-cocycle $\gamma \in Z^{2}\left(\bar{X}, F^{*}\right)$ and a simple $F K$-module $M$ in $f$ with $\operatorname{dim}_{F} M=m$ such that $f F X \cong\left(f_{\gamma} \bar{X}\right)_{m}$ and $f F N \cong\left(F_{\gamma_{1}} \bar{N}\right)_{m}$, where $\gamma_{1}$ is the restriction of $\gamma$ to $\bar{N}$.

(e) There are central extensions

$$
1 \rightarrow C \rightarrow X^{*} \rightarrow \bar{X} \rightarrow 1, \quad 1 \rightarrow C \rightarrow N^{*} \rightarrow \bar{N} \rightarrow 1,
$$

such that $C$ is a cyclic $p^{\prime}$-group with order $|C|$ equal to the order of the cocycle $\gamma$, and $X^{*}$ contains a p-block $B_{2}^{*}$ with defect group $P^{*} \cong P$ and Brauer correspondent $b_{2}^{*}$ in $N^{*}=N_{X} *\left(P^{*}\right)$ with $B \cong\left(B_{2}^{*}\right)_{m}$ and $b \cong\left(b_{2}^{*}\right)_{m}$.

(f) $X^{*}$ has a nonnormal T.I. Sylow p-subgroup $P^{*}$. which is neither cyclic nor a generalized quaternion 2-group, and its center $Z\left(X^{*}\right)=C=O_{p^{\prime}}\left(X^{*}\right)$ is cyclic.

Proof. (a) is an immediate consequence of Proposition 1.2.

By Proposition 1.2, $K=O_{p^{\prime}}(X) \leq N=N_{X}(P)$. If $\varphi$ is any modular irreducible character in $B$, then $\left.\varphi\right|_{N}$ has an irreducible constituent in $b$, by [11, Theorem 7.8, p. 131]. Therefore there is a block $f$ of $F K$ which is covered by $B$ and also by its Brauer correspondent $b$ in $N$. Let $T=T_{X}(f)=\{x \in$ $\left.X \mid x f x^{-1}=f\right\}$ be the inertial subgroup of $f$ in $X$. Then $T_{N}=T_{N}(f)=T \cap N$ is the inertial subgroup of $f$ in $N$. Now $P \leq T$ since $O_{p^{\prime}}(X) \leq N_{X}(P)$ implies that $P$ centralizes $O_{p^{\prime}}(X)$. By Fong's first reduction theorem [11, p. 197], there exists a unique $p$-block $B_{1}$ of $T$ with defect group $\delta\left(B_{1}\right)={ }_{T} P={ }_{X} \delta(B)$ such that $B=B_{1}^{X}$, and the block ideals of $B$ and $B_{1}$ have the same block invariants. Let $b_{1}$ be the Brauer correspondent of $B_{1}$ in $T_{N}=N_{T}(P)$. Then $b=b_{1}^{N}$ and $\delta(b)=P=\delta\left(b_{1}\right)$ by Theorem 1 of $\left[17, \mathrm{p}\right.$. 34]. Therefore, $b$ and $b_{1}$ have the same block invariants by Fong's first reduction theorem. Since $H \leq T$ by Proposition 1.2(f), both assertions (b) and (c) hold.

For the proof of the remaining assertions we assume that $X=T$.

Thus the block idempotent $f$ of $F K$ is central in $X$. As $p \nmid|K|$ the block ideal $f F K$ contains up to isomorphism only one simple $F K$-module $M$. Let $m=\operatorname{dim}_{F} M$. Then $f F K \cong(F)_{m}$. Since $\bar{X}=X / K$, Lemma I of [16, p. 1778] asserts that there exists a 2-cocycle $\gamma \in Z^{2}\left(\bar{X}, F^{*}\right)$ such that $f F X \cong\left(F_{\gamma} \bar{X}\right)_{m}$. By Proposition 1.2, $K=O_{p^{\prime}}(X) \leq N_{X}(P)$. Hence $N / K=\bar{N}=N_{\bar{X}}(\bar{P})$, and $f F N \cong\left(F_{\gamma_{1}} \bar{N}\right)_{m}$, where $\gamma_{1}$ is the restriction of $\gamma$ to $\bar{N}$. Thus (d) holds.

The construction of the central extension $1 \rightarrow C \rightarrow X^{*} \rightarrow \bar{X} \rightarrow 1$ with respect to the cocycle $\gamma \in Z^{2}\left(\bar{X}, F^{*}\right)$ is due to Fong (see [11]). As $C$ is a central cyclic subgroup of $X^{*}$ with $p^{\prime}$-order, the normalizer $N^{*}=N_{X^{*}}\left(P^{*}\right)$ of 
a Sylow $p$-subgroup $P^{*}$ of $X^{*}$ is a central extension

$$
1 \rightarrow C \rightarrow N^{*} \rightarrow \bar{N} \rightarrow 1
$$

with respect to the cocycle $\gamma_{1} \in Z^{2}\left(\bar{N}, F^{*}\right)$. Furthermore, $P^{*} \cong P^{*} C / C \cong$ $\bar{P} \cong P$, and $P^{*}$ is a nonnormal T.I. Sylow $p$-subgroup of $X^{*}$ which is neither cyclic nor a generalized quaternion 2-group. From (d), Theorem 2.5 of Cliff [7], and Theorem 2 of Okuyama and Tsushima [17] follows the existence of a $p$-block $B_{2}^{*}$ of $X^{*}$ with defect group $P^{*}$ and Brauer correspondent $b_{2}^{*}$ in $N^{*}$ such that

$$
B \cong\left(B_{2}^{*}\right)_{m} \text { and } b \cong\left(b_{2}^{*}\right)_{m}
$$

Clearly the center of $X^{*}$ is $Z\left(X^{*}\right)=C=O_{p^{\prime}}\left(X^{*}\right)$. This completes the proof.

Lemma 3.4. Let $X$ be a finite group with a nonnormal T.I. Sylow p-subgroup $P$. Suppose that $P$ is neither cyclic nor a generalized quaternion 2-group. Let $H=O^{p^{\prime}}(X)$ and $U=O^{p}(H)$.

If $H \neq U$, then all assertions of Proposition 3.1 hold.

Proof. Let $P$ be a Sylow $p$-subgroup of $X, N=N_{X}(P), K=O_{p^{\prime}}(X), L=$ $K U$ and $P_{2}=P \cap L$. Then by Proposition $1.2 P_{2}$ is isomorphic to a Sylow $p$-subgroup $P_{1}$ of the nonabelian simple group $G=U / O_{p^{\prime}}(U) \simeq L / O_{p^{\prime}}(L)$.

As $H \neq U$ we know that $P_{1}$ is cyclic by Proposition $1.2(\mathrm{~h})$, and either $p=3$ and $G=\operatorname{PSL}_{2}(8)$, or $p=5$ and $G={ }^{2} B_{2}\left(2^{5}\right)$. Furthermore, Proposition 1.2 asserts that $X / L$ is isomorphic to a subgroup of $\operatorname{Out}(G)$, which by the Atlas [8, pp. 6 and 77] is cyclic of order $p=3$ or 5 , respectively. As $P$ is not cyclic it follows in either of the two cases that $X / L \simeq \operatorname{Out}(G)$. Hence $X=H K$ by Proposition 1.2.

Now $G$ has a trivial Schur multiplier by [8, pp. 6 and 77]. Hence $Z(U)=$ 1 by Proposition 1.2(i). Therefore $G=U$ and $L=K \times U$. There exists $g \in X$ which induces a field automorphism of order $p$ by conjugation on $U$. Since $g^{p} \in K$ and $(|K|, p)=1$, there exists $k \in K$ with $(g k)^{p}=1$. Hence $X=K \times U\langle g k\rangle$. So the blocks $B$ of $X$ with positive defect and their Brauer correspondents $b$ in $N$ have the same block invariants as the principal blocks $B_{0}$ of $X$ and $b_{0}$ of $N$, respectively. Thus we may assume that $K=1$, $X=\operatorname{Aut}(G), B=B_{0}$ and $b=b_{0}$.

Let $e$ be the inertial index of the principal block $B_{1}$ of $G$, and let $b_{1}$ be the Brauer correspondent in $N_{1}=N_{G}\left(P_{1}\right)$. Then $e=2$ or 4 if $p=3$ or 5, respectively. As $P_{1}$ is cyclic, it follows from [11, pp. 277 and 278] that $k\left(B_{1}\right)=k_{0}\left(B_{1}\right)=\left(p^{2}-1\right) / e+e=k_{0}\left(b_{1}\right)=k\left(b_{1}\right)$ and $l\left(B_{1}\right)=l\left(b_{1}\right)=e$. Furthermore, $N$ is a semidirect product of the cyclic group $P_{1}$ or order $p^{2}$ and a cyclic group $Y$ of order $p e$. Since $b_{1}$ has $e$ one-dimensional and $\left(p^{2}-1\right) / e$ $e$-dimensional irreducible characters, and since the one-dimensional ones are also the $e$ modular irreducible characters of $N_{1}$, it follows that $b=F N$ has $p e+p+1$ ordinary irreducible characters with multiplicities and degrees $p e \times 1$, $p \times e$ and $1 \times p e$. Therefore $k(b)=p e+p+1, k_{0}(b)=p e+p$ and $l(b)=e$. 
On the other hand the character table of $X$ given in [8, pp. 6 and 77] shows that $k(B)=p e+p+1, k_{0}(B)=p e+p$ and $l(B)=e$. This completes the proof.

In [8, p. xxiii] two groups $X$ and $Y$ are called isoclinic if their centers $Z(X)$ and $Z(Y)$ can be enlarged so as to get isomorphic groups. This means that $X$ and $Y$ can be embedded into a larger group $W$ in such a way that $W=Z(W) * X=Z(W) * Y$, where $Z(W) * X$ denotes the central product of $X$ and $Z(W)$ with amalgamated subgroup $Z(X)=Z(W) \cap X$.

A finite group $X$ is a stem group if its commutator group $X^{\prime}$ contains the center $Z(X)$.

Suppose that $X$ satisfies the hypotheses of Proposition 3.1. Let $W \triangleleft X$ with $O^{p^{\prime}}(X) \leq W$. We wish to show that in proving Proposition 3.1 we may assume either that $X$ is an almost simple group or that $X$ and $Z(X)$ have a very specific structure as described below in Lemma 3.7. In the argument, it will be necessary to establish that if an element of $X$ induces by conjugation an outer automorphism of $W$ of order $m$ (modulo $\operatorname{Inn}(W)$ ), then there exists $y \in X$ which induces this automorphism and such that $y^{m} \in W$ (and not just that $\left.y^{m} \in W Z(X)\right)$. However, in order to produce such $y$, we must replace $X$ by an isoclinic group. Lemma 3.5 shows that this replacement is allowable in the proof of 3.1. The basic construction is given in Lemma 3.6. Given that $X / W Z(X)$ is cyclic of order $m$, we construct a suitable abelian group $V$ and element $y$ in $X * V$. Then $Y:=\langle W, y\rangle$ is shown to be isoclinic to $X$, and $y^{m} \in W$. This method is applied several times in the proof of Lemma 3.7 to yield a stem group which is isocolinic to $X$, and which is either almost simple or has the precise structure given in that lemma. Proposition 3.8 examines this alternative structure.

The following well-known lemma is stated without proof.

Lemma 3.5. Let $X$ be a group with $Z(X)$ a $p^{\prime}$-group, let $V$ be an abelian $p^{\prime}$ group with $Z(X) \leq V$, and let $A=X * V$ be a central product where $V \cap X=$ $Z(X)$. Then each $p$-block $B$ of $X$ is covered by $|V: Z(X)| p$-blocks $B^{\prime}$ of $A$. Moreover, $B$ and $B^{\prime}$ have the same defect groups and block invariants, and so have their Brauer correspondents $b$ and $b^{\prime}$, respectively.

Lemma 3.6. Let $X$ be a finite group with $H=O^{p^{\prime}}(X)$ such that $O_{p^{\prime}}(X)=$ $Z(X)=C_{X}(H)$ is cyclic. Let $W \geq H$ be a normal subgroup of $X$ such that $X / W Z(X)$ is a cyclic group of order $m$.

Then there exists a cyclic $p^{\prime}$-group $V \geq Z(X)$, and an element $y \in X * V$ such that

(a) $y^{m} \in W$ and $Y / W \cong X / W Z(X)$, where $Y=\langle W, y\rangle$.

(b) $H=O^{p^{\prime}}(X)=O^{p^{\prime}}(Y)$.

(c) $Z(Y)=V \cap W=Z(W)=C_{Y}(W)=O_{p^{\prime}}(Y)$.

(d) $A=X * V=Y * V$. 
Proof. Certainly $Z(X) \leq C_{X}(W) \leq C_{X}(H) \leq Z(X)$. Let $u \in X$ generate $X / W Z(X)$. Since $Z(X)=C_{X}(W), u$ induces an outer automorphism via conjugation on $W$ and $m$ is its order modulo inner automorphisms of $W$. Now $u^{m}=h z$ for some $h \in W, z \in Z(X)$, and $(p, m)=1$. There is a cyclic $p^{\prime}$-group $V \geq Z(X)$ containing an element $v$ such that $v^{m}=z$. Let $A$ be the central product $X * V$ with amalgamated subgroup $Z(X)=X \cap V$. Then $Z(A)=V=O_{p^{\prime}}(A), O^{p^{\prime}}(A)=O^{p^{\prime}}(X)=H \leq W$ and $A / W V \cong X / W Z(X)$.

In $A$ let $y=u v^{-1}$. Then $y$ generates $A / W V$, and $y^{m}=\left(u v^{-1}\right)^{m}=$ $u^{m} v^{-m}=h z z^{-1}=h \in W$.

Let $Y=\langle W, y\rangle \leq A$. Then $Y$ is normal in $A$. Hence $H=O^{p^{\prime}}(X)=$ $O^{p^{\prime}}(Y)$. As $v \in V=Z(A), y$ induces the same outer automorphism on $W$ as does $u$. In particular, $y$ has order $m$ modulo inner automorphisms of $W$, and $y^{i} w$ does not centralize $W$ for all $w \in W$ and $1 \leq i<m$. Hence

$$
C_{Y}(W)=Z(W)=W \cap Z(X)=W \cap V=V \cap Y=Z(Y) .
$$

Thus $O_{p^{\prime}}(Y)=O_{p^{\prime}}(A) \cap Y=V \cap Y=Z(Y)$, and

$$
|Y V|=\frac{|Y||V|}{|Y \cap V|}=\frac{m|W||V|}{|Z(W)|}=\frac{|X||V|}{|Z(X)|}=|X V| .
$$

Hence $A=Y * V=X * V$.

Lemma 3.7. Let $X$ be a finite group with a noncyclic T.I. Sylow p-subgroup such that $G=H / Z(H)$ is simple, where $H=O^{p^{\prime}}(X)$. Suppose that $K=O_{p^{\prime}}(X)=$ $Z(X)=C_{X}(H)$ is cyclic.

Then there exists a cyclic $p^{\prime}$-group $V$ and a stem group $W$ containing $H$ with $X * V=W * V$, such that either $W / H Z(W)$ is cyclic and $W$ is almost simple, or one of the following exceptional cases occurs:

(a) $G \in\left\{\operatorname{PSL}_{2}\left(p^{n}\right), \mathrm{PSU}_{3}\left(p^{2 n}\right)\right\}, H=O^{p^{\prime}}(W)$. There exists an element $y \in W$ which by conjugation on $H$ induces an outer diagonal automorphism $\gamma$ of $G$ of order $m \neq 1$ (modulo $\operatorname{Inn}(G)$ ), and there exists an element $t \in W$ which by conjugation on $H$ induces an outer field automorphism $\tau: x \rightarrow x^{p^{r}}$ of order $k=n r^{-1}$ or $k=2 n r^{-1}$ according as $G=\operatorname{PSL}_{2}\left(p^{n}\right)$ or $G=\operatorname{PSU}_{3}\left(p^{2 n}\right)$. Furthermore, $y^{m} \in H, y^{t}=y^{p^{r}} z$ for some $z \in Z(X)$ with $z^{m}=1, Y=$ $H\langle y, z\rangle \triangleleft W=\langle Y, t\rangle$, and $W / Y \cong X / H\langle u\rangle Z(X) \cong\langle\tau\rangle$, where $u$ is any element of $X$ which induces $\gamma$ by conjugation on $H$.

(b) $p=3, G=\operatorname{PSL}_{3}(4), H=O^{p^{\prime}}(W)$. There exists an element $y \in W$ which by conjugation on $H$ induces the outer graph-field automorphism $\gamma$ of $G$ of order $m=2$, and there exists an element $t \in W$ which by conjugation on $H$ induces the field automorphism $\tau$ of $G$, with $\tau^{m}=1$. Furthermore, $y^{2} \in Z(H)$, $y^{t}=y z$ for some $z \in Z(X)$ with $z^{m}=1, Y=H\langle y, z\rangle \triangleleft W=\langle Y, t\rangle$ and $W / Y \cong X / H\langle u\rangle Z(X) \cong\langle\tau\rangle$, where $u$ is any element of $X$ which induces $\gamma$ by conjugation on $H$. 
Moreover, in both of the cases (a) and (b), either $W$ is almost simple, or $Z(H)=1, z$ has order $m, Z(W)=\langle z\rangle$ and $Y=H\langle y\rangle \times\langle z\rangle$.

Proof. By Proposition 1.2, $X / H Z(X) \leq \operatorname{Out}(G)$, and $H=O^{p^{\prime}}(X)$ is perfect. If $\bar{X}=X / H Z(X)$ is cyclic, then by Lemma 3.6 there exists a cyclic $p^{\prime}$-group $V$ and an almost simple stem group $W$ such that $X * V=W * V$.

Therefore we may assume that $\bar{X}$ is not cyclic. Then

$$
G \in\left\{\operatorname{PSL}_{2}\left(p^{n}\right), \operatorname{PSU}_{3}\left(p^{2 n}\right), \operatorname{PSL}_{3}(4)\right\}
$$

by Proposition 1.3.

In the first two cases it follows from [8] that $G$ has an outer diagonal automorphism $\gamma \neq 1$ of order $m \in\{2,3\}$ and an outer field automorphism $\tau: x \rightarrow x^{p^{r}}$ of order $k=n r^{-1}$ or $k=2 n r^{-1}$ if $G=\operatorname{PSL}_{2}\left(p^{n}\right)$ or $G=$ $\mathrm{PSU}_{3}\left(p^{2 n}\right)$, respectively, such that $\bar{X}=\langle\gamma, \tau\rangle \leq \operatorname{Out}(G)$. If $G=\operatorname{PSL}_{3}(4)$, then by [8] $G$ has an outer graph-field automorphism $\gamma$ of order $m=2$ and an outer field automorphism $\tau \neq 1$ such that $\bar{X}=\langle\gamma, \tau\rangle \leq \operatorname{Out}(G)$ is a Klein four group. In each case there is an element $u \in X$ such that the conjugation of $H$ by $u$ induces $\gamma$ modulo inner automorphisms. Thus $u^{m}=h z_{1}$ for some $h \in H$ and $z_{1} \in Z(X)$. As $Z(X)=O_{p^{\prime}}(X)$ is cyclic, there exists a cyclic $p^{\prime}$-group $V_{1} \geq Z(X)$ containing an element $v$ with $v^{m}=z_{1}$. In $X_{1}=V_{1} * X$ let $y=u v^{-1}$. Then $y^{m}=h$ and $X_{1} / H V_{1} \cong X / H Z(X)$, $O^{p^{\prime}}\left(X_{1}\right)=H, O_{p^{\prime}}\left(X_{1}\right)=Z\left(X_{1}\right)=C_{X_{1}}(H)$ by the proof of Lemma 3.6. Furthermore, $H\langle y\rangle V_{1} \triangleleft X_{1}$ and $H\langle y\rangle V_{1} / H V_{1} \cong\langle\gamma\rangle \leq \operatorname{Out}(G)$. Therefore, we may assume that $X=X_{1}, y^{m}=h \in H_{1}=H$, and $H\langle y\rangle Z(X)<X$. The unique lifts of $\tau$ and $\gamma$ to $H$ are denoted by the same letters.

By the structure of $X / H Z(X) \leq \operatorname{Out}(G)$ there is a $t \in X$ which generates $X / H\langle y\rangle Z(X)$ and induces the automorphism $\tau$ on $H$.

Now let $G \in\left\{\operatorname{PSL}_{2}\left(p^{n}\right), \operatorname{PSU}_{3}\left(p^{2 n}\right)\right\}$. Then $y^{m}$ is a diagonal matrix of $H$ or an image of such if $H$ is simple, because $H$ is a central factor group of a group of matrices over a finite field $G F(q)$. Furthermore, $y$ acts on $H$ as conjugation by a diagonal matrix $M$ with entries over some finite extension of $G F(q)$. Note that $M^{\tau}=M^{p^{r}}$, and that $y^{i}$ operates on $H$ as conjugation by $M^{i}$. Let $h \in H$. Then

$$
\begin{aligned}
h^{\left(y^{t}\right)} & =h^{t^{-1} y t}=\left(h^{\tau^{-1}}\right)^{y t}=\left(M^{-1} h^{\tau^{-1}} M\right)^{t} \\
& =\left(M^{-1}\right)^{\tau} h^{\tau^{-1} \tau} M^{\tau}=M^{-p^{r}} h M^{p^{r}}=h^{\left(y^{p^{\prime}}\right)} .
\end{aligned}
$$

Therefore $y^{t}=y^{p^{r}} z$ for some $z \in Z(X)=C_{X}(H)$. Hence $\left(y^{m}\right)^{p^{r}}=\left(y^{m}\right)^{\tau}=$ $\left(y^{m}\right)^{t}=y^{m p^{r}} z^{m}$. Thus $z^{m}=1$.

Let $Y=H\langle y, z\rangle$. Then $Y \triangleleft X$. Since $m$ is a prime number, $Z(H) \leq Z(X)$, $|Z(H)| \in\{1, m\}$, and $Z(X)$ is cyclic, it follows that either $z \in Z(H), Y=$ $H\langle y\rangle$ and $Z(H)=Z(Y)$; or $Z(H)=1, H=G$ is simple, and $Y=H\langle y\rangle \times\langle z\rangle$ where $z \neq 1$. In each case, $X / Y Z(X)$ is isomorphic to a cyclic group $\langle\tau\rangle$ of 
outer field automorphisms of $G$. Therefore Lemma 3.6 asserts the existence of a cyclic $p^{\prime}$-group $V_{2}$ and an element $t_{1} \in X * V_{2}$ such that $W=\left\langle Y, t_{1}\right\rangle$ satisfies $V_{2} * W=V_{2} * X, Y \triangleleft W, t_{1}^{k} \in Y, y^{t_{1}}=y^{p^{r}} z, H=O^{p^{\prime}}(X)=O^{p^{\prime}}(W)$, $C_{W}(H)=Z(W)=Z(Y)=O_{p^{\prime}}(W)$, and $W / Y \cong X / H\langle y\rangle Z(X) \cong\langle\tau\rangle$.

In the first case we have $\langle z\rangle \leq Z(W)=Z(H) \leq H=H^{\prime} \leq W^{\prime}$. Hence $W$ is an almost simple stem group satisfying all the conditions of assertion (a). In the second case, $\langle z\rangle=Z(Y)=Z(W)$, and $y^{t_{1}} \equiv y^{ \pm 1} z(\bmod H)$. If $y^{t_{1}} \equiv y z(\bmod H)$ then $t_{1}^{-1} y t_{1} y^{-1} \equiv z(\bmod H)$, whence $\langle z\rangle \leq W^{\prime}$. If $m=3$ and $y^{t_{1}} \equiv y^{-1} z(\bmod H)$, then $p^{r} \equiv-1(\bmod 3)$ and hence $(y z)^{t_{1}}=y^{p^{r}} z^{2}=$ $(y z)^{p^{r}}$. So in this situation, we may reduce to the first case by replacing $y$ with $y z$. We may assume, then, that the second case implies $\langle z\rangle=Z(W) \leq W^{\prime}$, hence $W$ is a stem group with all the properties stated in (a).

Let $G=\mathrm{PSL}_{3}(4)$. Let $y \in X$ induce a graph-field automorphism $\gamma$ of order 2 on $H$. As $y^{2} \in H, y^{2} \in Z(H)$. Let $t$ be as above. Since $\operatorname{Out}(G) / O_{p}(\operatorname{Out}(G))$ is a Klein four group, $\tau$ and $\gamma$ commute. Thus $y^{t}$ and $y$ yield the same action on $H$. Therefore $y^{t}=y z$ for some $z \in Z(X)=C_{X}(H)$. Let $Y=H\langle y, z\rangle$. Then $Y \triangleleft X$. As $Z(H) \leq Z(X)$ and $y^{2} \in Z(H)$, we have

$$
y^{2}=\left(y^{2}\right)^{t}=\left(y^{t}\right)^{2}=(y z)^{2}=y^{2} z^{2} .
$$

Thus $z^{2}=1$. If $Z(H) \neq 1$, then $z \in Z(H)$, because $Z(X)$ is cyclic, $Z(H) \leq$ $Z(X)$, and $Z(H)$ is a 2-group by Proposition 1.2 and [8]. In case $Z(H)=1$, $Y=H\langle y\rangle \times\langle z\rangle$. In each case, $X / Y Z(X)$ is isomorphic to a cyclic group $\langle\tau\rangle$ of outer field automorphisms of $G$ of order $k=2$. Applying now Lemma 3.6 as above, assertion (b) and the remaining part of the last assertion follow. This completes the proof.

Proposition 3.8. Suppose the assertions of Proposition 3.1 hold for all almost simple groups with a T.I. Sylow p-subgroup. Then they hold for all finite groups $X$ with a T.I. Sylow p-subgroup.

Proof. Let $X$ be a finite group with a T.I. Sylow $p$-subgroup $P$. Let $B$ be a $p$ block of $X$ with defect group $P$ and Brauer correspondent $b$ in $N=N_{X}(P)$. Then by Lemma 3.2 we may assume that $P$ is neither cyclic nor a generalized quaternion 2-group and that $P$ is not normal in $X$. Let $H=O^{p^{\prime}}(X), U=$ $O^{p}(H), K=O_{p^{\prime}}(X)$ and $L=K U$. By Lemma 3.4 we may assume that $H=U$. Now Lemma 3.3(a) and Proposition 1.2 imply that $G=U / O_{p^{\prime}}(U)=$ $H / K \cap H \cong L / K$ is a nonabelian simple group with a noncyclic Sylow $p$ subgroup. As the Fong correspondences described in Lemma 3.3(c) and (e) preserve the block invariants $k(B)$ and $k_{0}(B)$, Lemma 3.3(f) asserts that we also may assume that $O_{p^{\prime}}(X)=K=Z(X)$ is a cyclic $p^{\prime}$-group.

Suppose that $X$ is not almost simple. Then by Lemma 3.7 there exists a cyclic $p^{\prime}$-group $V$ and a stem group $W$ such that $X * V=A=W * V$, where $W=\langle Y, t\rangle$ and $Y=G\langle y\rangle \times\langle z\rangle \triangleleft W$ have all the properties described 
in Lemma 3.7. In particular, $Z(W)=Z(Y)=\langle z\rangle$ has order $m=2$ or 3 if $G \in\left\{\mathrm{PSL}_{2}\left(p^{n}\right), \mathrm{PSL}_{3}(4)\right\}$ or $G=\mathrm{PSU}_{3}\left(p^{2 n}\right)$, respectively.

Certainly $P$ is a T.I. Sylow $p$-subgroup in $A$ and therefore also in $W$. By Lemma 3.5 there is a block correspondence between $X$ and $W$ which preserves defect groups, Brauer correspondence, and block invariants. Hence we may assume that $X=W$.

Let $G=\operatorname{PSL}_{3}(4)$. Then Lemma 3.7(b) asserts that $p=3$ and $G=O^{p^{\prime}}(X)$. Furthermore, there exists an involution $y \in X$ which by conjugation on $G$ induces an outer graph-field automorphism $\gamma$ of $G$ of order $m=2$, and there exists an element $t \in X$ which by conjugation on $G$ induces an outer field automorphism $\tau$ of $G$ of order $k=2$ such that $y^{t}=y z$. Let $\bar{X}=X /\langle z\rangle$. Then, by [8, p. 24], $C_{\bar{X}}(\bar{P})=\langle\bar{y}\rangle \bar{P}$. So $O_{p^{\prime}}\left(C_{X}(P)\right)=\langle y, z\rangle=O_{p^{\prime}}(N)$ contains three conjugacy classes of $N=N_{X}(P)$. Hence $X$ has three blocks of positive defect, two of which have $Z(X)=\langle z\rangle$ in their kernel, and therefore belong to the almost simple group $X / Z(X) \leq \operatorname{Aut}(G)$. Thus we may assume that $B$ is the only faithful block of $X$. It covers two faithful blocks $B_{1}$ and $B_{2}$ of $Y=G\langle y\rangle \times\langle z\rangle$ which both have the same block invariants as the principal 3-block $B_{0}$ of the almost simple group $G\langle y\rangle$. From $y^{t}=y z$ follows that $B=B_{1}^{X}=B_{2}^{X}$. Hence $B$ has the same block invariants as $B_{0}$. Similarly we can show that the block invariants of $b$ and the Brauer correspondent $b_{0}$ of $B_{0}$ are equal.

Let $G \in\left\{\operatorname{PSL}_{2}(q), \operatorname{PSU}_{3}\left(q^{2}\right)\right\}$, where $q=p^{n}$. Then Lemma 3.7(a) asserts that $G=O^{p^{\prime}}(X)$. Furthermore, there exists an element $y \in X$ which by conjugation on $G$ induces an outer diagonal automorphism $\gamma$ of $G$ of order $m \neq 1$, and there exists an element $t \in X$ which by conjugation on $G$ induces an outer field automorphism $\tau: x \rightarrow x^{p^{r}}$ of order $k=n r^{-1}$ or $k=2 n r^{-1}$, if $G=\operatorname{PSL}_{2}(q)$ or $G=\operatorname{PSU}_{3}\left(q^{2}\right)$, respectively. Moreover, $y^{m} \in G$ and $y^{t}=y^{p^{r}} z$. Let $E=G\langle y\rangle$. Then $E \cong \operatorname{PGL}_{2}(q)$ or $E \cong \mathrm{PU}_{3}\left(q^{2}\right), Y=$ $E \times\langle z\rangle \triangleleft X=\langle Y, t\rangle$, and $X / Y$ is a cyclic group of order $k$. Hence $t^{k}=z^{u}$ for some $0 \leq u<m$, as $t^{k}$ acts trivially on $G$.

Furthermore, the automorphism which $t$ induces on $Y /\langle z\rangle \cong E$ is an extension of $\tau$, which is also denoted by $\tau$; in fact it is a field automorphism of $E=G\langle y\rangle$ such that $y^{\tau}=y^{p^{r}}$. Now $E$ and $\bar{X}=X /\langle z\rangle \cong E\langle\tau\rangle \leq \operatorname{Aut}(G)$ are almost simple groups.

By the character tables of $E \in\left\{\mathrm{PGL}_{2}(q), \mathrm{PU}_{3}\left(q^{2}\right)\right\}$ (see [22, 10], respectively), the principal block $B_{0}(E)$ of $E$ is the only block of $E$ with positive defect. It is also the principal block $B_{0}(Y)$ of $Y=E \times\langle z\rangle$. Let $\lambda$ be a faithful linear complex character of $\langle z\rangle$. Then $\left\{B_{i}(Y)=B_{0}(Y) \otimes \lambda^{i} \mid 0 \leq i<m\right\}$ is the set of all blocks of $Y$ with positive defect.

Let $N=N_{X}(P)$ and $N_{1}=N_{Y}(P)$. Since $O_{p^{\prime}}(N)=\langle z\rangle, X$ also has $m$ blocks $B_{i}=B_{i}(X), 0 \leq i<m$, with positive defect. We may choose notation so that $B_{i}(Y)$ is only covered by $B_{i}$. Furthermore, $\left[B_{i}(Y)\right]^{t}=B_{i}(Y)$. 
Let $b_{i}\left(N_{1}\right)$ be the Brauer correspondent of $B_{i}(Y)$ in $N_{1}=N_{Y}(P)$, and $b_{i}=$ $b_{i}(N)$ the one of $B_{i}$ in $N=N_{X}(P)$. Then $\left\{b_{i}\left(N_{1}\right)=b_{0}\left(N_{1}\right) \otimes \lambda^{i} \mid 0 \leq i<m\right\}$ is the set of all blocks of $N_{1}$, where $b_{0}\left(N_{1}\right)$ denotes the principal block of $N_{1}$. Since $E$ is almost simple and $Y=E \times\langle z\rangle$, it follows for each $0 \leq i<m$ that the blocks $B_{i}(Y)$ and $b_{i}\left(N_{1}\right)$ have the same block invariants. By Brauer's first main theorem, $b_{i}$ is the unique block of $N$ covering $b_{i}\left(N_{1}\right)$. From Proposition 1.2 (c) follows that $N_{Y}(P)=N_{G}(P)\langle y, z\rangle \triangleleft N_{X}(P)=\left\langle N_{Y}(P), t\right\rangle$. For $0 \leq i<$ $m$, each coset $G y^{i}$ of $G$ in $E$ is a union of conjugacy classes of $Y$, and any conjugacy class of $Y$ is of the form $C z^{j}$, where $C$ is a $Y$-class contained in some coset $G y^{i}$. Let $g \in G$ and $0 \leq i, j<m$. Then $\left(g y^{i} z^{j}\right)^{t}=g^{t} y^{i p^{r}} z^{i} z^{j}=$ $\left(g y^{i}\right)^{\tau} z^{i+j}$. Therefore the action of $t$ on the conjugacy classes of $Y$ is given by

$$
\left(C z^{j}\right)^{t}=C^{\tau} z^{i+j}
$$

Hence $C z^{j}$ with $C \subseteq G y^{i}$ is fixed by $t$ if and only if $i=0$ and $C^{\tau}=C \subseteq G$. As $N=\left\langle N_{1}, t\right\rangle$, the same assertions hold for the action of $t$ on the classes of $N_{1}$, where $G$ is replaced by $N_{2}=N_{G}(P)$.

Since $y \in E$ induces a diagonal automorphism of $G$ by conjugation, the $p$-regular classes of $E$ contained in $G$ are precisely the $p$-regular conjugacy classes of $G$. It follows that the numbers of $p$-regular classes of $Y$ and $N_{1}$ fixed by $t$ equal $m$ times the numbers of $p$-regular classes of $G$ and $N_{2}=N_{G}(P)$ fixed by $\tau$, respectively. Applying Lemma 3.6 of [11, p. 146] and Brauer's permutation lemma we get $f(Y, t)=m f(G, \tau)$ and $f\left(N_{1}, t\right)=m f\left(N_{2}, \tau\right)$, where $f(W, t)$ and $f(W, \tau)$ denote the numbers of modular irreducible characters of a group $W$ fixed by $t$ and $\tau$, respectively.

Each modular irreducible character $\psi$ of $Y$ is of the form $\psi=\varphi \lambda^{i}$, where $\varphi$ is a uniquely determined modular irreducible character of $E$, and $0 \leq i<m$. Let $\rho$ be the linear character of $E / G$ such that $\rho(y)=\lambda(z)$. Then for every $Y$-class $C$ with $C \subseteq G y^{k}, 0 \leq k<m$, it follows from (*) that

$$
\psi^{t^{-1}}(C)=\psi\left(C^{\tau} z^{k}\right)=\varphi\left(C^{\tau}\right) \lambda^{i}\left(z^{k}\right)=\varphi^{\tau^{-1}}(C) \rho^{i}(C) .
$$

Hence $\psi^{t}=\psi$ if and only if $\varphi=\varphi^{\tau^{-1}} \rho^{i}$. The same condition holds also in $N_{1}$.

The Steinberg character St of $G$ has $m^{2}$ irreducible extensions St $\rho^{k} \lambda^{i}$, $0 \leq i, k<m$, to $Y=E \times\langle z\rangle$. From the character tables of $\operatorname{PGL}_{2}(q)$ and $\mathrm{PU}_{3}\left(q^{2}\right)$ of [22] and [10] follows that $m$ of these $m^{2}$ blocks of defect zero of $Y$ are fixed by $t$. For any block $B$ in a group $W$ let $f(B, t)$ and $f(B, \tau)$ be the numbers of modular irreducible characters fixed by $t$ and $\tau$, respectively. As the principal block $B_{0}(X)$ of $X$ is the principal block of the almost simple group $\bar{X}=X /\langle z\rangle \cong E\langle\tau\rangle$ whose Brauer correspondent is isomorphic to $b_{0}(N)$, the conclusion of Proposition 3.1 for almost simple groups implies that $f\left(B_{0}(Y), t\right)=f\left(b_{0}\left(N_{1}\right), t\right)$. Since $G$ is almost simple, Proposition 3.1 
asserts that $f(G, \tau)=f\left(N_{2}, \tau\right)+1$. Recall that $f(Y, t)=m f(G, \tau)$ and $f\left(N_{1}, t\right)=m f\left(N_{2}, t\right)$. Hence

$$
\begin{aligned}
f(Y, t) & =m+f\left(B_{0}(Y), t\right)+\sum_{i=1}^{m-1} f\left(B_{i}(Y), t\right)=m+m f\left(N_{2}, \tau\right) \\
& =m+f\left(b_{0}\left(N_{1}\right), t\right)+\sum_{i=1}^{m-1} f\left(b_{i}\left(N_{1}\right), t\right) .
\end{aligned}
$$

If $m=3$, then $B_{1}(Y)$ and $B_{2}(Y)$ are complex conjugate. Thus $f\left(B_{i}(Y), t\right)=$ $f\left(b_{i}\left(N_{1}\right), t\right)$ for $0 \leq i<m$, in any case. The same argument applies for each power of $t$. Hence Proposition 2.3 asserts that $l\left(B_{i}\right)=l\left(b_{i}\right)$ for all $0 \leq i<m-1$. Thus $k\left(B_{i}\right)=k\left(b_{i}\right)$ for all $i$ by Lemma 2.1.

If $G=\operatorname{PSL}_{2}(q)$, then all irreducible characters of $X$ have height zero. So we may assume that $G=\operatorname{PSU}_{3}\left(q^{2}\right)$ and that $m=3$ divides $q+1$. For any block $B(U)$ of any group $U$ let $\operatorname{rh}(B(U))$ be the number of irreducible characters $\chi$ of $B(U)$ with positive height. By the character table [21] we have $\operatorname{rh}\left(B_{0}(G)\right) \stackrel{1}{=}(q+1)$ for the principal block $B_{0}(G)$ of $G$. Using the character table [10] of $E=G\langle y\rangle=\mathrm{PU}_{3}\left(q^{2}\right)$ and Theorem 2.14 of [11, p. 102], it is easy to see that each irreducible character of $B_{0}(G)$ with positive height is fixed by $y$ and has therefore $m$ irreducible extensions $\chi \rho^{j}, 0 \leq j<m-1$, to $B_{0}(E)=B_{0}(Y)$. Furthermore, $\operatorname{rh}\left(B_{0}(E)\right)=q+1$. From [10] follows also that the raised height characters of $N_{E}(P)$ occur in triples $\xi, \xi \rho, \xi \rho^{2}$, where $\xi$ is an irreducible character of positive height in $N_{E}(P)$ and $\xi_{\mid N_{2}}=\xi \rho_{\mid N_{2}}^{j}$, where $N_{2}=N_{G}(P)$.

For any group $W$ and any block $B$ of $W$ let $\operatorname{rh}(W, \alpha)$ and $\operatorname{rh}(B, \alpha)$ be the numbers of irreducible characters with positive height of $W$ and $B$ fixed by the automorphism $\alpha$ of $W$, respectively. Applying again Proposition 3.1 to the almost simple groups $G, G\langle\tau\rangle$ and $E, E\langle\tau\rangle$, we obtain that

$(* * *)$

$$
\begin{gathered}
\operatorname{rh}(G, \tau)=\operatorname{rh}\left(N_{2}, \tau\right), \\
\operatorname{rh}\left(B_{0}(Y), t\right)=\operatorname{rh}\left(B_{0}(E), t\right)=\operatorname{rh}\left(b_{0}\left(N_{1}\right), t\right) .
\end{gathered}
$$

Each irreducible character $\psi$ with positive height of $B_{i}(Y)=B_{0}(Y) \otimes \lambda^{i}$ is of the form $\psi=\chi \otimes \lambda^{i}$, where $\chi$ is a uniquely determined character of positive height in $B_{0}(Y)$. As above it follows that $\psi^{t}=\psi$ if and only if $\chi=\chi^{\tau^{-1}} \rho^{i}$. In particular, $\left(\chi_{\mid G}\right)^{\tau}=\chi_{\mid G}$, and $\left.\bar{\chi}^{\tau^{-1}}=\overline{\left(\chi^{\tau^{-1}}\right)}=\overline{\left(\chi \rho^{-i}\right.}\right)=\bar{\chi} \rho^{i}$, where $\bar{\chi}$ denotes the complex conjugate character of $\chi$. Thus $\left(\chi \otimes \lambda^{i}\right)^{t}=\chi \otimes \lambda^{i}$ implies that $\left(\bar{\chi} \otimes \lambda^{i}\right)^{t} \neq \bar{\chi} \otimes \lambda^{i}$ for $i=1,2$, but $\left.\left(\bar{\chi} \otimes \lambda^{i}\right)^{t}\right|_{G}=\left.\bar{\chi} \otimes \lambda^{i}\right|_{G}$.

The same arguments apply for the raised height characters of $N_{1}=N_{Y}(P)$. It follows from this discussion, $(* *)$ and $(* * *)$ that for $i=1$ or 2

$$
\begin{aligned}
\operatorname{rh}\left(B_{i}(Y), t\right) & =\frac{1}{2}\left[3 \operatorname{rh}(G, \tau)-\operatorname{rh}\left(B_{0}(Y), t\right)\right] \\
& =\frac{1}{2}\left[3 \operatorname{rh}\left(N_{2}, \tau\right)-\operatorname{rh}\left(b_{0}\left(N_{1}\right), t\right)\right]=\operatorname{rh}\left(b_{i}\left(N_{1}\right), t\right) .
\end{aligned}
$$


Hence $k_{0}\left(B_{i}\right)=k\left(B_{i}\right)-\operatorname{rh}\left(B_{i}\right)=k\left(b_{i}\right)-\operatorname{rh}\left(b_{i}\right)=k_{0}\left(b_{i}\right)$ for $0 \leq i<m-1$. This completes the proof.

\section{Conjugacy classes of $\mathrm{SL}_{2}(q), \mathrm{SU}_{3}\left(q^{2}\right),{ }^{2} B_{2}(q),{ }^{2} G_{2}(q)$ FIXED BY AUTOMORPHISMS}

In this section we study the action of field and diagonal automorphisms on the conjugacy classes of a finite quasi-simple group of Lie type $G$ with a T.I. Sylow $p$-subgroup, and the action of field automorphisms on the conjugacy classes of an almost simple extension of $G$ by an outer diagonal automorphism. This will enable us to extend the assertions of the main theorem from simple groups $G$ of Lie type defined over finite fields with characteristic $p$ to extensions of $G$ described in Proposition 3.8.

Lemma 4.1. Let $\tilde{G}$ be a simple and simply connected algebraic group with an endomorphism $\sigma$ such that $G=\tilde{G}_{\sigma}$ is a finite group realized over the field $\operatorname{GF}\left(q^{i}\right)$ with $q^{i}=p^{k i}$ elements, where $i \in\{1,2,3\}$. Let $\tau$ be the field automorphism of $\tilde{G}$ obtained from the map $x \rightarrow x^{p}$ on a suitable algebraically closed field. Let $\delta$ be the graph automorphism of $\tilde{G}$ such that either $\delta$ has order $i$ and $\sigma=\delta \tau^{k}$ or, if $G \in\left\{{ }^{2} B_{2}(q),{ }^{2} G_{2}(q),{ }^{2} F_{4}(q)\right\}, \delta^{2}=\tau, i=1, k$ is odd and $\sigma=\delta \tau^{(k-1) / 2}$. For every $r \mid k i$, define $w_{r}=(r-1) / 2$ if $\delta^{2}=\tau, w_{r}=r$ if $\sigma=\delta \tau^{k}$ and $r \mid k$, and $w_{r}=r i^{-1}$ if $\sigma=\delta \tau^{k}$ and $r \nmid k$. Put $t=0$ if $\delta^{2}=\tau$, $t=r w_{r}^{-1}$ otherwise.

Then the number of semisimple conjugacy classes of $G=\tilde{G}_{\sigma}=G\left(p^{i k}\right)$ fuxed by $\tau^{r}$ equals the number of all semisimple conjugacy classes of the finite group $\tilde{G}_{\delta \tau^{w_{r}}}=G\left(p^{i w_{r}}\right)$ which are fixed by $\delta^{t}$.

Proof. For $H \in\{\tilde{G}, G\}$ let $\mathscr{S}(H)$ be the set of all semisimple conjugacy classes of $H$. If $\rho$ is an endomorphism of $H$, then let $\mathscr{S}(H)_{\rho}=\{C \in$ $\left.\mathscr{S}(H) \mid C^{\rho}=C\right\}$. Corollary 3.10 of Springer-Steinberg [5, p. 197] asserts that $\hat{C} \rightarrow C=\hat{C} \cap \tilde{G}_{\sigma}$ is a bijection from $\mathscr{S}(\tilde{G})_{\sigma}$ onto $\mathscr{S}(G)$. It follows that this correspondence induces a bijection between $\mathscr{S}(G)_{\tau^{r}}$ and $\mathscr{S}(\tilde{G})_{\sigma} \cap \mathscr{S}(\tilde{G})_{\tau^{r}}$, the set of fixed points of $\mathscr{S}(\tilde{G})$ under the action of the group $\left\langle\sigma, \tau^{r}\right\rangle$.

If $G$ is of type ${ }^{2} B_{2}(q),{ }^{2} G_{2}(q)$ or ${ }^{2} F_{4}(q)$, i.e. if $\delta^{2}=\tau$ and $\sigma=\delta \tau^{(k-1) / 2}$, we may write $r j=k$ for some odd integer $j$. Then $(k-1) / 2=r(j-1) / 2$ $+(r-1) / 2$ implies that $\delta \tau^{(r-1) / 2}=\sigma \tau^{-r(j-1) / 2},\left(\delta \tau^{(r-1) / 2}\right)^{2}=\tau^{r}$ and $\left(\delta \tau^{(r-1) / 2}\right)^{j}=\delta \tau^{(k-1) / 2}$, hence that $\left\langle\sigma, \tau^{r}\right\rangle=\left\langle\delta \tau^{(r-1) / 2}\right\rangle$. So

$$
\left|\mathscr{S}(G)_{\tau^{r}}\right|=\left|\mathscr{S}(\tilde{G})_{\delta \tau^{(r-1) / 2}}\right|=\left|\mathscr{S}\left(\tilde{G}_{\delta \tau^{(r-1) / 2}}\right)\right|,
$$

again by Corollary 3.10 of [5].

If $G$ is untwisted $(\delta=1)$ or is twisted of type ${ }^{2} A_{n}\left(q^{2}\right),{ }^{2} D_{n}\left(q^{2}\right),{ }^{2} E_{6}\left(q^{2}\right)$, or ${ }^{3} D_{4}\left(q^{3}\right)$, then $\sigma=\delta \tau^{k}$. As $r \mid k i$ we have $k \equiv \varepsilon w_{r}(\bmod r)$ by definition of $w_{r}$, where $\varepsilon=1$, unless $\tilde{G}_{\sigma}={ }^{3} D_{4}\left(q^{3}\right)$ in which case $i=3$ and $\varepsilon= \pm 1$. 
Note that $\tilde{G}_{\delta \tau^{e w_{r}}}=G\left(p^{i w_{r}}\right)$ because the cases $\varepsilon=1$ and $\varepsilon=-1$ yield the same Steinberg triality. Write $k=m r+\varepsilon w_{r}$. Then $\delta \tau^{k} \tau^{-m r}=\delta \tau^{\varepsilon w_{r}}$ implies that $\left\langle\sigma, \tau^{r}\right\rangle=\left\langle\delta \tau^{\varepsilon w_{r}}, \tau^{r}\right\rangle$. Hence

$$
\left|\mathscr{S}(G)_{\tau^{r}}\right|=\left|\mathscr{S}(\tilde{G})_{\delta \tau^{\ell w_{r}}} \cap \mathscr{S}(\tilde{G})_{\tau^{r}}\right|=\left|\mathscr{S}\left(G\left(p^{i w_{r}}\right)\right)_{\tau^{r}}\right|=\left|\mathscr{S}\left(G\left(p^{i w_{r}}\right)\right)_{\delta^{t}}\right|
$$

by a final application of Corollary 3.10 of [5].

Remark. With the notation of Lemma 4.1 and its proof, the assertion of this result can be restated as follows.

(a) If $G$ is untwisted or of type ${ }^{2} B_{2}(q),{ }^{2} G_{2}(q),{ }^{2} F_{4}(q)$, then $\left|\mathscr{S}(G)_{\tau^{r}}\right|=$ $\left|\mathscr{S}\left(G\left(p^{r}\right)\right)\right|$.

(b) If $G$ is a twisted group of Lie type ${ }^{2} A_{n}\left(q^{2}\right),{ }^{2} D_{n}\left(q^{2}\right),{ }^{2} E_{6}\left(q^{2}\right)$, or ${ }^{3} D_{4}\left(q^{3}\right)$, then $\left|\mathscr{S}(G)_{\tau^{r}}\right|=\left|\mathscr{S}\left(G\left(p^{r}\right)\right)\right|$, if $w_{r}=r / i$ with $i \in\{2,3\} ;\left|\mathscr{S}(G)_{\tau^{r}}\right|=$ $\left|\mathscr{S}\left(G\left(p^{i r}\right)\right)_{\delta^{\prime}}\right|$, if $w_{r}=r$.

Lemma 4.2. Let $G \in\left\{\mathrm{SL}_{2}\left(p^{n}\right), \mathrm{SU}_{3}\left(p^{2 n}\right),{ }^{2} B_{2}\left(2^{n}\right),{ }^{2} G_{2}\left(3^{n}\right)\right\}$, where $p$ is a prime and $n=2 k+1$ for the exceptional groups of Lie type. Let $\tau$ be a field automorphism of $G$ of order $n$ or $2 n$, if $G=\mathrm{SU}_{3}\left(p^{2 n}\right)$. For every $r \mid n$ or $r \mid 2 n$ let $s_{r}$ be the number of semisimple conjugacy classes of $G$ fixed by $\tau^{r}$. Then $s_{r}=p^{r}$.

Proof. If $G$ is $\mathrm{SL}_{2}\left(p^{n}\right),{ }^{2} B_{2}\left(2^{n}\right)$ or ${ }^{2} G_{2}\left(3^{n}\right)$, then Lemma 4.1 and the subsequent remark (a) yield that $s_{r}=\left|\mathscr{S}\left(G\left(p^{r}\right)\right)\right|$. This equals $p^{r}$ by Theorem 3.7.6 of Carter [6] (or by [9, 23, 25]). If $G$ is $\mathrm{SU}_{3}\left(p^{2 n}\right)$ and $r \nmid n$ then Lemma 4.1 and remark (b) imply $s_{r}=\left|\mathscr{S}\left(\mathrm{SU}_{3}\left(p^{2(r / 2)}\right)\right)\right|$ which again equals $p^{r}$ by Theorem 3.7.6 of [6], or by [21]. If $G$ is $\mathrm{SU}_{3}\left(p^{2 n}\right)$ and $r \mid n$, then $s_{r}=\left|\mathscr{S}\left(\mathrm{SU}_{3}\left(p^{2 r}\right)\right)_{\delta}\right|$ by Lemma 4.1 and remark (b), where $\delta$ is the inverse transpose automorphism. So $s_{r}=p^{r}$ by [21, p. 487].

The following notation is used for Proposition 4.3 below and its proof. Let $p$ be a prime and $q=p^{k}, k$ a positive integer. Let $\sigma$ be a primitive element of $\operatorname{GF}\left(q^{2}\right)$. Let $\alpha=\sigma^{q+1}$ (so that $\alpha \in \mathrm{GF}(q)$ has order $q-1$ ), $\beta=\sigma^{q-1}$, $\nu=\sigma^{(q+1) / 2}, \mu=\sigma^{(q-1) / 2}$, if $q$ is odd.

Let $H=\operatorname{SL}_{2}(q)$ and $d=(p-1,2)$. If $d=2$ let $x=\left(\nu^{-1}\right)$, so that conjugation by $x$ generates the outer diagonal automorphisms of ${ }^{\nu}$. Let $E=E(q)=H\langle x\rangle$. Note that $x^{2}=\left(\alpha_{\alpha}^{-1}\right) \in H$ and $Z(E)=Z(H)=\{ \pm I\}$, where $I=\left(\begin{array}{ll}1 & \\ & 1\end{array}\right)$.

Let $\tau$ denote the field automorphism of $\mathrm{GL}_{2}\left(q^{2}\right)$ obtained from $c \rightarrow c^{p}$, all $c \in G F\left(q^{2}\right)$. Both $H$ and $E$ are stable under $\tau$, as is the Sylow $p$-subgroup $P=\left\{\left(\begin{array}{ll}1 & 0 \\ c & 1\end{array}\right): c \in \mathrm{GF}(q)\right\}$ of $H$ (and of $E$ ). For any $\tau$-stable section $X$ of $H$ or of $E$, and integer $r$, let $s_{r}(X)$ denote the number of semisimple ( $p$ regular) classes of $X$ which are fixed by $\tau^{r}$. In any result about $E$ or $Z(H)$ in Proposition 4.3 below, we assume of course that $d=2$. 
Proposition 4.3. For any positive integer $r \mid k$,

(a) $s_{r}(H)=p^{r}=s_{r}\left(N_{H}(P)\right)+1$;

(b) $s_{r}(E)=p^{r}+1=s_{r}\left(N_{E}(P)\right)+2=s_{r}(E / Z(E))=s_{r}\left(N_{E}(P) / Z(E)\right)+2$;

(c)

$s_{r}(H / Z(H))=s_{r}\left(N_{H}(P) / Z(H)\right)+1= \begin{cases}\left(p^{r}-1\right) / 2+1 & \text { if } k / r \text { is odd, } \\ p^{r} & \text { if } k / r \text { is even; }\end{cases}$

(d) E has two p-blocks of defect zero, both of which have $Z(E)$ in their kernel and are fixed by $\tau^{r}$.

The proof is broken into several steps.

Step 1. The semisimple conjugacy classes of $H$ are as follows: there are $(q+d-1) / 2$ classes $C_{1}^{(j)}$, with representative $\left(\alpha^{-j}{ }_{\alpha^{j}}\right)$ in $\mathrm{GL}_{2}\left(q^{2}\right)$ for $1 \leq$ $j \leq q-1$ (and with $\left.C_{1}^{(j)}=C_{1}^{(-j)}\right)$; and there are $(q+1-d) / 2$ classes $C_{2}^{(j)}$, with representative $\left({ }^{\beta^{-j}} \beta^{j}\right)$ in $\mathrm{GL}_{2}\left(q^{2}\right)$ for $1 \leq j \leq q+1$ with $j \not \equiv 0$ $(\bmod (q+1) / d)$ and with $C_{2}^{(j)}=C_{2}^{(-j)}$. In particular, distinct classes have distinct sets of eigenvalues.

Proof. This is clear from [9, p. 228].

Step 2. The semisimple conjugacy classes of $E$ are as follows: there are $q$ classes $A_{1}^{(j)}$, with representative $\left(\nu^{-j}{ }_{\nu^{j}}\right)$ in $\mathrm{GL}_{2}\left(q^{2}\right)$ for $1 \leq j \leq 2(q-1)$ and with $A_{1}^{(j)}=A_{1}^{(-j)}$; and there are $q$ classes $A_{2}^{(j)}$, with representative $\left(\begin{array}{ll}\mu^{-j} & \\ \mu^{j}\end{array}\right)$ in $\mathrm{GL}_{2}\left(q^{2}\right)$ for $1 \leq j \leq 2(q+1)$ with $j \not \equiv 0(\bmod q+1)$ and $A_{2}^{(j)}=A_{2}^{(-j)}$.

Proof. Let $J=\mathrm{GL}_{2}(q)$. Since $x=\left({ }^{1}{ }_{\alpha}\right)\left(\nu^{-1} I\right) \in J\langle\nu I\rangle$ and $\operatorname{det}(\nu I)=\alpha$ has order $q-1$, we have that $J\langle\nu I\rangle=E\langle\nu I\rangle$. Denote the semisimple classes of $E$ and $J$ by $\left\{A_{j}\right\}$ and $\left\{C_{n}\right\}$, respectively. Then the semisimple classes of $J\langle\nu I\rangle$ are $\left\{A_{j} \nu^{i} \mid 0 \leq i \leq q-2\right\}=\left\{C_{n} \nu^{i} \mid i=0,1\right\}$. The list of semisimple classes of $J$ in $[22$, p. 226] now implies the result.

Step 3. Except for the classes $A_{1}^{((q-1) / 2)}$ and $A_{2}^{((q+1) / 2)}$ (which have the same eigenvalue set $\left\{\sigma^{ \pm\left(q^{2}-1\right) / 4}\right\}$, i.e. 4th roots of unity in $\left.\operatorname{GF}\left(q^{2}\right)\right)$, distinct semisimple classes of $E$ have distinct eigenvalue sets.

Proof. This is clear from Step 2.

Step 4. $s_{r}(H)=p^{r}=s_{r}\left(N_{H}(P)\right)+1$.

Proof. $s_{r}(H)=p^{r}$ by Lemma 4.2. Now $N_{H}(P)=P\langle g\rangle$, where $g=\left(\begin{array}{ll}\alpha^{-1} & \end{array}\right)$ has order $q-1$. The elements $g^{j}, 1 \leq j \leq q-1$, are representatives for the $q-1$ semisimple conjugacy classes of $N_{H}(P)$. So $\tau^{r}$ fixes the $N_{H}(P)$-class of $g^{j}$ if and only if $\left(g^{j}\right)^{p^{r}}=g^{j}$, i.e. $g^{j\left(p^{r}-1\right)}=1$. Since $p^{r}-1 \mid q-1$, there are $p^{r}-1$ solutions $j$. 
Step 5. $s_{r}(E)=p^{r}+1=s_{r}\left(N_{E}(P)\right)+2$.

Proof. We have that $E \leq \mathrm{SL}_{2}\left(q^{2}\right)$, and except for the two classes $A_{1}^{\left(j_{1}\right)}$ and $A_{2}^{\left(j_{2}\right)}\left(j_{1}=(q-1) / 2 ; j_{2}=(q+1) / 2\right)$ with eigenvalues $\sigma^{ \pm\left(q^{2}-1\right) / 4}$, exactly one of which is in $H$, the semisimple classes of $E$ remain distinct in $\operatorname{SL}_{2}\left(q^{2}\right)$ by Step 3. Furthermore, the semisimple classes of $H$ do not fuse in $E$. By Step 4, $\tau^{r}$ fixes $p^{r}$ semisimple classes both of $H$ and of $\operatorname{SL}_{2}\left(q^{2}\right)$. Since it fixes $A_{1}^{\left(j_{1}\right)}$ and $A_{2}^{\left(j_{2}\right)}$, it therefore fixes $p^{r}+1$ semisimple classes of $E$.

Now $N_{E}(P)=P\langle x\rangle$, where $x=\left(\nu_{\nu}^{-1}\right)$. The elements $x^{j}, 1 \leq j \leq$ $2(q-1)$, represent the semisimple classes of $N_{E}(P)$, and the class of $x^{j}$ is fixed by $\tau^{r}$ if and only if $x^{j\left(p^{r}-1\right)}=1$. There are $p^{r}-1$ solutions $j$.

Step 6. If $k / r$ is odd then $s_{r}(H / Z(H))=\left(p^{r}-1\right) / 2+1=s_{r}\left(N_{H}(P) / Z(H)\right)+1$. Proof. Let $X$ denote either $H$ or $N_{H}(P)$. If $C$ is a semisimple class of $X$ such that $C^{\tau^{r}}=-C$, then $C^{\tau^{2 r}}=C$. It follows that $2 \mid \operatorname{order}\left(\tau^{r}\right)$ as an automorphism of $X$. But $\operatorname{order}\left(\tau^{r}\right)=k / r$, a contradiction. So $\tau^{r}$ fixes only the images in $X / Z(H)$ of those semisimple classes of $X$ already fixed by $\tau^{r}$. The preimages of a class of $X / Z(H)$ have the form $C,-C$, which are distinct unless $X=H$ and $C$ has eigenvalues $\sigma^{ \pm\left(q^{2}-1\right) / 4}$. The result follows from Step 4.

Step 7. If $k r^{-1}$ is even then

$$
s_{r}(H / Z(H))=p^{r}=s_{r}\left(N_{H}(P) / Z(H)\right)+1 .
$$

Proof. Since here $p$ is odd,

$$
2 \mid 1+p^{r}+p^{2 r}+\cdots+p^{(k / r-1) r}=\left(p^{k}-1\right) /\left(p^{r}-1\right) .
$$

Let $n=\left(p^{k}-1\right) / 2\left(p^{r}-1\right)$. Let $s=\left(\alpha_{\alpha^{n}}^{-n}\right)$. Then $s^{2} \in \operatorname{SL}_{2}\left(p^{r}\right) \leq H$. Note that $H \geq E\left(p^{r}\right)=\mathrm{SL}_{2}\left(p^{r}\right)\langle s\rangle$, and that $E\left(p^{r}\right)$ is stabilized by $\tau^{r}$. Now $\alpha^{n p^{r}}=-\alpha^{n}$ implies that $s^{\tau^{r}}=-s$. As $\tau^{r}$ is trivial on $\operatorname{SL}_{2}\left(p^{r}\right)$, it follows that $C^{\tau^{r}}= \pm C$ for every semisimple class $C$ of $E\left(p^{r}\right)$. Only the two classes of $E\left(p^{r}\right)$ with eigenvalues $\sigma^{ \pm\left(q^{2}-1\right) / 4}$ fuse in $H$, by Step 3, and $E\left(p^{r}\right)$ has $2 p^{r}$ semisimple classes, by Step 2 . So there are at least $2 p^{r}-1$ semisimple classes of $H$ which are fixed modulo $Z(H)$ by $\tau^{r}$. Hence $\tau^{r}$ fixes at least $\left(2 p^{r}-2\right) / 2+1=p^{r}$ semisimple classes of $H / Z(H)$. By Lemma 3.6 of Feit $\left[11\right.$, p. 146] and by Brauer's permutation lemma, $\tau^{r}$ fixes at least $p^{r}$ irreducible Brauer characters of $H / Z(H)$. But $\tau^{r}$ fixes a total of $p^{r}$ irreducible Brauer characters of $H$, by Step 4. So $s_{r}(H / Z(H))=p^{r}$.

Now $N_{H}(P)=P\langle g\rangle$, where $g=\left(\alpha_{\alpha}^{-1}\right)$, and $\tau^{r}$ fixes the class of $g^{j}$ modulo $Z(H)$ if and only if $g^{j p^{r}}= \pm g^{j}$, which is equivalent to $\alpha^{2 j\left(p^{r}-1\right)}=1$. Since here $2\left(p^{r}-1\right) \mid p^{k}-1$, there are $2\left(p^{r}-1\right)$ solutions $j$, hence $p^{r}-1$ semisimple classes of $N_{H}(P) / Z(H)$ fixed by $\tau^{r}$. 
Step 8. $s_{r}(E / Z(E))=p^{r}+1=s_{r}\left(N_{E}(P) / Z(E)\right)+2$.

Proof. Let $m=(q-1) /\left(p^{r}-1\right)$. Let $u=x^{m}=\left(\nu_{\nu^{m}}^{-m}\right)$, so that $u^{2}=$ $\left(\begin{array}{cc}\alpha^{-m} & \\ \alpha^{m}\end{array}\right)$, where $\alpha^{m}$ has order $p^{r}-1$. Then $\mathrm{SL}_{2}\left(p^{r}\right)\langle u\rangle=E\left(p^{r}\right) \leq E(q)$, and $E\left(p^{r}\right)$ is stable under $\tau$. Since $\tau^{r}$ is trivial on $\operatorname{SL}_{2}\left(p^{r}\right)$ and $u^{\tau^{r}}=u^{p^{r}}=$ $-u, \tau^{r}$ fixes every semisimple class of $E\left(p^{r}\right)$ modulo $Z(E)$. The semisimple classes of $E\left(p^{r}\right)$ do not fuse in $E(q)$, by Steps 2 and 3, so $\tau^{r}$ fixes at least $2 p^{r}$ semisimple classes of $E(q)=E$ modulo $Z(E)$. Since the two classes of $E$ with eigenvalues $\sigma^{ \pm\left(q^{2}-1\right) / 4}$ are not equal modulo $Z(E), \tau^{r}$ fixes at least $\left(2 p^{r}-2\right) / 2+2=p^{r}+1$ semisimple classes, and hence Brauer characters of $E / Z(E)$. Step 5 now implies $s_{r}(E / Z(E))=p^{r}+1$.

In $N_{E}(P)$, the semisimple class of $x^{j}, 1 \leq j \leq 2(q-1)$, is fixed modulo $Z(E)$ if and only if $x^{2 j\left(p^{r}-1\right)}=1$. There are $2\left(p^{r}-1\right)$ solutions for $j$, and hence $p^{r}-1$ fixed semisimple classes of $N_{E}(P) / Z(E)$.

Step 9. Part (d) holds.

Proof. From the character table for $J=\mathrm{GL}_{2}(q)$ in [22, p. 227], and the fact that $J\langle\nu I\rangle=E\langle\nu I\rangle$, it follows that $E$ has two characters $\chi^{(u)}$ for $u=1,2$ such that $\chi^{(u)}(1)=q$ and the value of $\chi^{(u)}$ on any element $g_{j} \in A_{2}^{(j)}$ (as in Step 2$)$ is $-(-1)^{u j}$. The $\chi^{(u)}$ comprise the only blocks of defect zero. It follows that $\chi^{(1) \tau^{r}} \neq \chi^{(2)}$.

The proof of Proposition 4.3 is complete.

The following notation is used for the statement and proof of the next two propositions, 4.4 and 4.5. Let $p$ be a prime and $q=p^{k}, k$ a fixed positive integer. In the field $\operatorname{GF}\left(q^{6}\right), \gamma$ is an element of order $q^{3}+1 ; \rho=\gamma^{q^{2}-q+1}$ (so that $\rho \in \mathrm{GF}\left(q^{2}\right)$ has order $\left.q+1\right) ; \sigma$ is a primitive element of $\operatorname{GF}\left(q^{2}\right)$ with $\sigma^{q-1}=\rho ; \pi$ is a cube root of $\rho$ in $\operatorname{GF}\left(q^{6}\right)$; and if $q \equiv-1(\bmod 3)$, $\omega=\rho^{(q+1) / 3}$. Let $\theta \neq 0$ be any fixed element of $\operatorname{GF}\left(q^{2}\right)$ which is not in $\left\langle\sigma^{3}\right\rangle$.

Let $H=\mathrm{SU}_{3}\left(q^{2}\right)$ and $d=(q+1,3)$. If $d=3$, let

$$
x=\left(\begin{array}{lll}
\pi^{-1} & & \\
& \pi^{-1} & \\
& & \pi^{2}
\end{array}\right) .
$$

Then conjugation by $x$ generates the outer diagonal automorphisms of $H$. Let $E=E\left(q^{2}\right)=H\langle x\rangle$. Now

$$
x^{3}=\left(\begin{array}{lll}
\rho^{-1} & & \\
& \rho^{-1} & \\
& & \rho^{2}
\end{array}\right) \in H,
$$

and $Z(E)=Z(H)=\langle\omega I\rangle$, where

$$
I=\left(\begin{array}{lll}
1 & & \\
& 1 & \\
& & 1
\end{array}\right) .
$$


Let $\tau$ denote the field automorphism of $\mathrm{GL}_{3}\left(q^{6}\right)$ obtained from $c \rightarrow c^{p}$, all $c \in \mathrm{GF}\left(q^{6}\right) . H$ and $E$ are stable under $\tau$. For any $\tau$-stable section $X$ of $H$ or of $E$, and any positive integer $r$, let $c_{r}(X), s_{r}(X), h z_{r}(X), d z_{r}(X)$ denote the number of conjugacy classes, number of semisimple ( $p$-regular) classes, number of irreducible characters of height zero, number of $p$-blocks of defect zero, respectively, which are fixed by $\tau^{r}$. There is a Sylow $p$-subgroup $P$ of $H$ (hence of $E$, when $d=3$ ) which is stable under $\tau$.

If $d=3$, let $\delta$ denote any proper outer diagonal automorphism of $H$. For $r$ any positive integer, and $X$ any section of $H$ stable under $\delta$ and $\tau$, let $s_{r}^{\delta}(X), c_{r}^{\delta}(X), h z_{r}^{\delta}(X)$ denote the number of semisimple classes, number of conjugacy classes, number of irreducible characters of height zero, respectively, which are fixed by $\delta \tau^{r}$.

Note that $s_{2 k}^{\delta}(X), c_{2 k}^{\delta}(X), h z_{2 k}^{\delta}(X)$ give the numbers of fixed points under the action of $\delta$. Let $N=N_{H}(P)$.

Proposition 4.4. For any positive integer $r \mid 2 k$, the numbers $s_{r}, c_{r}, h z_{r}, d z_{r}$ of fixed points of $\tau^{r}$ are given in Table $1 \quad(d=3$ in all cases referring to $E$ or $Z(H))$.

Proposition 4.5. Let $d=3$ and $r \mid 2 k$. Then

(a) $s_{r}^{\delta}(X)=s_{r}(X)$ for $X=H, N, H / Z(H), N / Z(H)$;

(b) $\quad c_{r}^{\delta}(H)=c_{r}^{\delta}(N)+1= \begin{cases}c_{r}(H) & \text { if } r \mid k \\ c_{r}(H)-9 & \text { if } r+k ;\end{cases}$

(c) $\quad c_{r}^{\delta}(H / Z(H))=c_{r}^{\delta}(N / Z(H))+1= \begin{cases}c_{r}(H / Z(H)) & \text { if } r \mid k, \\ c_{r}(H / Z(H))-3 & \text { if } r \nmid k ;\end{cases}$

(d) $\quad h z_{r}^{\delta}(H)=h z_{r}^{\delta}(N)+1= \begin{cases}p^{r}+1 & \text { if } r \mid k, \\ p^{r} & \text { if } r+k ;\end{cases}$

(e) $h z_{r}^{\delta}(H / Z(H))=h z_{r}^{\delta}(N / Z(H))+1= \begin{cases}p^{r}+1 & \text { if } r \mid k, \\ p^{r} & \text { if } r+k \text { and } 3 \mid \frac{2 k}{r}, \\ \frac{p^{r}-1}{3}+1 & \text { if } r+k \text { and } 3+\frac{2 k}{r} .\end{cases}$

Proof of Proposition 4.4. Step 1. The conjugacy classes of $H$ are as in Table 2 (note that each semisimple class is uniquely determined by its eigenvalues).

Proof. See [21, p. 487].

Step 2. The conjugacy classes of $E$ are as in Table 3.

Proof. Let $J=\mathrm{GU}_{3}\left(q^{2}\right)$ and $y=\pi I$. Since

$$
x=\left(\begin{array}{lll}
1 & & \\
& 1 & \\
& & \rho
\end{array}\right) y^{-1} \in J\langle y\rangle
$$

and $\operatorname{det} y=\rho$ has order $q+1$, it follows that $J\langle y\rangle=E\langle y\rangle$ and that $E$ contains precisely those elements of $J\langle y\rangle$ with determinant 1 . If we denote 
TABLE 1

\begin{tabular}{|c|c|c|}
\hline Group $X$ & $s_{r}(X)$ & $c_{r}(X)$ \\
\hline$H$ & $p^{r}$ & $\begin{array}{l}p^{r}+(2, p-1)+1 \text { if } r \mid k \\
p^{r}+p^{r / 2}+d^{2}+1 \text { if } r \nmid k\end{array}$ \\
\hline$N_{H}(P)$ & $p^{r}-1=s_{r}(H)-1$ & $\left.\begin{array}{l}p^{r}+(2, p-1) \text { if } r \mid k \\
p^{r}+p^{r / 2}+d^{2} \text { if } r \nmid k\end{array}\right\}=c_{r}(H)-1$ \\
\hline$H / Z(H)$ & $\begin{array}{l}p^{r} \text { if } r \mid k, \\
\text { or if } r+k \text { and } 3 \mid \frac{2 k}{r} \\
\frac{p^{r}-1}{3}+1 \text { if } r+k \\
\text { and } 3+\frac{2 k}{r}\end{array}$ & $\begin{array}{l}p^{r}+(2, p-1)+1 \text { if } r \mid k \\
\frac{p^{r}-1}{3}+\frac{p^{r / 2}+1}{3}+4 \\
\quad \text { if } r \nmid k \text { and } 3 \nmid \frac{2 k}{r} \\
p^{r}+p^{r / 2}+4 \\
\quad \text { if } r \nmid k \text { and } 3 \mid \frac{2 k}{r}\end{array}$ \\
\hline$N_{H}(P) / Z(H)$ & $s_{r}(H / Z(H))-1$ & $c_{r}(H / Z(H))-1$ \\
\hline$E$ & $\begin{array}{l}p^{r} \text { if } r \mid k, \\
p^{r}+2 \text { if } r+k\end{array}$ & $\begin{array}{l}p^{r}+(2, p-1)+1 \text { if } r \mid k \\
p^{r}+p^{r / 2}+6 \text { if } r \nmid k\end{array}$ \\
\hline$N_{E}(P)$ & $p^{r}-1$ & $\begin{array}{l}p^{r}+(2, p-1) \text { if } r \mid k \\
p^{r}+p^{r / 2}+3 \text { if } r \nmid k\end{array}$ \\
\hline$E / Z(E)$ & $s_{r}(E)$ & $\begin{array}{l}p^{r}+(2, p-1)+1 \text { if } r \mid k \\
p^{r}+p^{r / 2}+4 \text { if } r+k\end{array}$ \\
\hline$N_{E}(P) / Z(E)$ & $p^{r}-1=s_{r}\left(N_{E}(P)\right)$ & $\begin{array}{l}p^{r}+(2, p-1) \text { if } r \mid k \\
p^{r}+p^{r / 2}+1 \text { if } r \nmid k\end{array}$ \\
\hline$H$ & $\begin{array}{l}p^{r}+1 \text { if } r \mid k \\
p^{r}+d^{2} \text { if } r+k\end{array}$ & 1 \\
\hline$N_{H}(P)$ & $h z_{r}(H)-1$ & 0 \\
\hline$H / Z(H)$ & $\begin{array}{l}p^{r}+1 \text { if } r \mid k, \\
p^{r}+3 \text { if } r+k \text { and } 3 \mid \frac{2 k}{r} \\
\frac{p^{r}-1}{3}+4 \text { if } r+k \text { and } 3+\frac{2 k}{r}\end{array}$ & 1 \\
\hline$N_{H}(P) / Z(H)$ & $h z_{r}(H / Z(H))-1$ & 0 \\
\hline$E$ & $\begin{array}{l}p^{r}+1 \text { if } r \mid k \\
p^{r}+5 \text { if } r+k\end{array}$ & $\begin{array}{l}1 \text { if } r \mid k \\
3 \text { if } r \nmid k\end{array}$ \\
\hline$N_{E}(P)$ & $\begin{array}{l}p^{r} \text { if } r \mid k \\
p^{r}+2 \text { if } r+k\end{array}$ & 0 \\
\hline$E / Z(E)$ & $\begin{array}{l}p^{r}+1 \text { if } r \mid k \\
p^{r}+3 \text { if } r+k\end{array}$ & $\begin{array}{l}1 \text { if } r \mid k \\
3 \text { if } r \nmid k\end{array}$ \\
\hline$N_{E}(P) / Z(E)$ & $p^{r}$ & 0 \\
\hline
\end{tabular}


TABLE 2

\begin{tabular}{|c|c|c|c|}
\hline $\begin{array}{c}\text { Conjugacy } \\
\text { class }\end{array}$ & $\begin{array}{l}\text { Representative } \\
\left(\text { in } \mathrm{GL}_{3}\left(q^{6}\right)\right)\end{array}$ & Parameters & $\begin{array}{c}\text { Number of } \\
\text { classes }\end{array}$ \\
\hline$C_{1}^{(j)}$ & $\left(\begin{array}{ccc}\omega^{j} & & \\
& \omega^{j} & \\
& & \omega^{j}\end{array}\right)$ & $0 \leq j \leq d-1$ & $d$ \\
\hline$C_{2}^{(j)}$ & $\left(\begin{array}{ccc}\omega^{j} & & \\
1 & \omega^{j} & \\
& & \omega^{j}\end{array}\right)$ & $0 \leq j \leq d-1$ & $d$ \\
\hline$C_{3}^{(j, l)}$ & $\left(\begin{array}{ccc}\omega^{j} & & \\
\theta^{-l} & \omega^{j} & \\
& \theta^{l} & \omega^{j}\end{array}\right)$ & $0 \leq j, l \leq d-1$ & $d^{2}$ \\
\hline$C_{4}^{(j)}$ & $\left(\begin{array}{lll}\rho^{j} & & \\
& \rho^{j} & \\
& & \rho^{-2 j}\end{array}\right)$ & $\begin{array}{l}1 \leq j \leq q+1 \\
j \not \equiv 0(\bmod (q+1) / d)\end{array}$ & $q+1-d$ \\
\hline$C_{5}^{(j)}$ & $\left(\begin{array}{lll}\rho^{j} & & \\
1 & \rho^{j} & \\
& & \rho^{-2 j}\end{array}\right)$ & $\begin{array}{l}1 \leq j \leq q+1 \\
j \not \equiv 0(\bmod (q+1) / d)\end{array}$ & \\
\hline$C_{6}^{(j, l, m)}$ & $\left(\begin{array}{lll}\rho^{j} & & \\
& \rho^{l} & \\
& & \rho^{m}\end{array}\right)$ & $\begin{array}{l}1 \leq j, l, m \leq q+1 \\
j, l, m \text { distinct } \\
(\bmod q+1) \\
j+l+m \equiv 0 \\
(\bmod q+1)\end{array}$ & $\begin{array}{l}\frac{1}{6}(q+1)(q-2) \\
\quad+\frac{d}{3}\end{array}$ \\
\hline$C_{7}^{(j)}$ & $\left(\begin{array}{ccc}\rho^{j} & & \\
& \sigma^{j} & \\
& & \sigma^{-q j}\end{array}\right)$ & $\begin{array}{l}1 \leq j \leq q^{2}-1 \\
j \not \equiv 0(\bmod q-1) \\
\left(C_{7}^{(j)}=C_{7}^{(-q j)}\right)\end{array}$ & $\frac{1}{2}(q-2)(q+1)$ \\
\hline$C_{8}^{(j)}$ & $\left(\begin{array}{lll}\gamma^{(q+1) j} & & \\
& \gamma^{-(q+1) q j} & \\
& & \gamma^{(q+1) q^{2} j}\end{array}\right)$ & $\begin{array}{l}1 \leq j \leq q^{2}-q+1 \\
j \not \equiv 0 \\
\quad\left(\bmod \left(q^{2}-q+1\right) / d\right) \\
\left(C_{8}^{(j)}=C_{8}^{(-q j)}=C_{8}^{\left(q^{2} j\right)}\right)\end{array}$ & $\begin{array}{l}\frac{1}{3}\left(q^{2}-q\right. \\
\quad+1-d)\end{array}$ \\
\hline
\end{tabular}

the sets of conjugacy classes of $E$ and $J$ by $\left\{A_{j}\right\}$ and $\left\{C_{n}\right\}$, respectively, then the conjugacy classes of $J\langle y\rangle$ are given by $\left\{A_{j} y^{i} \mid 0 \leq i \leq q\right.$, all $\left.A_{j}\right\}=$ $\left\{C_{n} y^{i} \mid 0 \leq i \leq 2\right.$, all $\left.C_{n}\right\}$. These observations and the classification of the conjugacy classes of $J$ given by Ennola [10, p. 29], imply the result.

Step 3. Except for the three classes $A_{6}^{(a, 2 a, 3 a, 0)}$, where $a=(q+1) / 3$, and $A_{8}^{(a, i)}$, where $i=1$ or 2 and $a=q^{3}+1-i\left(q^{2}-q+1\right) / 3$ (which have the same eigenvalue set $\left.\left\{1, \omega, \omega^{2}\right\}\right)$, distinct semisimple ( $p$-regular) classes of $E$ have distinct eigenvalue sets. 
Substep 3.1. If $\gamma^{a} \pi^{i} \in\langle\pi\rangle$, where $1 \leq a \leq q^{3}+1,0 \leq i \leq 2, a \neq 0\left(\bmod q^{2}-\right.$ $q+1)$ and $a \equiv-i(\bmod q+1)$ then $i \neq 0$ and $\left\{\gamma^{a} \pi^{i}, \gamma^{-a q} \pi^{i}, \gamma^{a q^{2}} \pi^{i}\right\}=$ $\left\{1, \omega, \omega^{2}\right\}$.

Proof. If $\gamma^{a} \in\langle\pi\rangle$ then order $\left(\gamma^{a}\right) \mid 3(q+1)$, and hence $a=j\left(q^{2}-q+1\right) / 3$ for some integer $j$. Now $q^{2}-q+1=(q+1)(q-2)+3$ implies that $\left(q^{2}-q+1\right) / 3 \equiv 1$ $(\bmod q+1)$. So $a \equiv-i(\bmod q+1)$ yields $j \equiv-i(\bmod q+1)$. Put $j=$ $t(q+1)-i$ for some integer $t$. Then $a=(t(q+1)-i)\left(q^{2}-q+1\right) / 3$. So $q^{2}-q+1 \nmid a$ forces $i \neq 0$. Since $\gamma^{\left(q^{3}+1\right) / 3} \in\langle\omega\rangle$ and $\gamma^{-i\left(q^{2}-q+1\right) / 3} \in \pi^{-i}\langle\omega\rangle$, we have $\gamma^{a} \pi^{i} \in\langle\omega\rangle$. Also, $\gamma^{-a q} \pi^{i}=\left(\gamma^{a} \pi^{i}\right)^{-q} \pi^{(q+1) i}=\gamma^{a} \pi^{i} \omega^{i}$ and $\gamma^{a q^{2}} \pi^{i}=$ $\gamma^{a} \pi^{i} \omega^{2 i}$. The result follows.

Substep 3.2. If $1 \leq a, b \leq q^{3}+1, a, b \neq \equiv\left(\bmod q^{2}-q+1\right), 0 \leq i, j \leq 2$, $a \equiv-i(\bmod q+1), b \equiv-j(\bmod q+1)$ and

$$
\left\{\gamma^{a} \pi^{i}, \gamma^{-a q} \pi^{i}, \gamma^{a q^{2}} \pi^{i}\right\}=\left\{\gamma^{b} \pi^{j}, \gamma^{-b q} \pi^{j}, \gamma^{b q^{2}} \pi^{j}\right\},
$$

then either $i=j$ and $\left\{a,-a q, a q^{2}\right\} \equiv\left\{b,-b q, b q^{2}\right\}\left(\bmod q^{3}+1\right)$, or (up to permutation of indices) $i=1, a=q^{3}+1-\left(q^{2}-q+1\right) / 3, j=2, b=$ $q^{3}+1-2\left(q^{2}-q+1\right) / 3$ and both sets equal $\left\{1, \omega, \omega^{2}\right\}$.

Proof. If $i=j$ then we may assume that $\gamma^{a} \pi^{i}=\gamma^{b} \pi^{j}$, hence $a=b$ and the result follows. So we may assume that $\gamma^{a} \pi^{i}=\gamma^{b} \pi^{j}$ and $i \neq j$. Then $\gamma^{a-b}=\pi^{j-i}$ yields $\left(q^{2}-q+1\right) / 3 \mid a-b$.

If $\gamma^{-a q} \pi^{i}=\gamma^{-b q} \pi^{j}$ then $\pi^{i-j}=\gamma^{(a-b) q}=\pi^{(j-i) q}$, hence $\pi^{(j-i)(q+1)}=1$. So $i, j \in\{0,1,2\}$ implies that $i=j$, a contradiction. Thus $\gamma^{-a q} \pi^{i}=\gamma^{b q^{2}} \pi^{j}$ and $\gamma^{a q} \pi^{i q}=\gamma^{b q} \pi^{j q}$, hence

$$
\pi^{i(q+1)}=\gamma^{-a q} \pi^{i} \gamma^{a q} \pi^{i q}=\gamma^{b q^{2}} \pi^{j} \gamma^{b q} \pi^{j q}=\gamma^{b\left(q^{2}+q\right)} \pi^{j(q+1)} .
$$

Thus $\gamma^{b\left(q^{2}+q\right)} \in\langle\omega\rangle$, whence $\gamma^{b} \in\langle\pi\rangle$, therefore $\gamma^{a} \in\langle\pi\rangle$. Then Substep 3.1 implies that $i, j \neq 0$ and

$$
\left\{\gamma^{a} \pi^{i}, \gamma^{-a q} \pi^{i}, \gamma^{a q^{2}} \pi^{i}\right\}=\left\{1, \omega, \omega^{2}\right\}=\left\{\gamma^{b} \pi^{j}, \gamma^{-b q} \pi^{j}, \gamma^{b q^{2}} \pi^{j}\right\} .
$$

Now we may assume that $i=1$ and $\gamma^{a} \pi=1$, hence $\gamma^{a}=\pi^{-1}$. So one of $a,-a q, a q^{2}$ is congruent to $-\left(q^{2}-q+1\right) / 3\left(\bmod q^{3}+1\right)$. Then $j=2$ and $\gamma^{b} \pi^{2}=1$, hence one of $b,-b q, b q^{2}$ is congruent to $-2\left(q^{2}-q+1\right) / 3$ $\left(\bmod q^{3}+1\right)$.

Substep 3.3. There are no other repeated sets of eigenvalues among the semisimple classes of $E$.

Proof. The sets $\left\{\pi^{a}, \pi^{a}, \pi^{-2 a}\right\}$ and $\left\{\pi^{3 a+i}, \pi^{3 b+i}, \pi^{3 c+i}\right\}$, as parametrized in Step 2, are clearly all distinct. If $\sigma^{b} \pi^{i}=\pi^{u}$ for some $b, i$ as in Step 2 and some $u$, then $\operatorname{order}\left(\sigma^{b}\right) \mid\left(q^{2}-1,3(q+1)\right)=q+1$. Then $q-1 \mid b$, a contradiction. 
TABLE 3

\begin{tabular}{|c|c|c|c|}
\hline $\begin{array}{c}\text { Conjugacy } \\
\text { class }\end{array}$ & $\begin{array}{l}\text { Representative } \\
\left.\text { (in } \mathrm{GL}_{3}\left(q^{6}\right)\right)\end{array}$ & Parameters & $\begin{array}{l}\text { Number of } \\
\text { classes }\end{array}$ \\
\hline$A_{1}^{(a)}$ & $\left(\begin{array}{lll}\omega^{a} & & \\
& \omega^{a} & \\
& & \omega^{a}\end{array}\right)$ & $0 \leq a \leq 2$ & 3 \\
\hline$A_{2}^{(a)}$ & $\left(\begin{array}{ccc}\omega^{a} & & \\
1 & \omega^{a} & \\
& & \omega^{a}\end{array}\right)$ & $0 \leq a \leq 2$ & 3 \\
\hline$A_{3}^{(a)}$ & $\left(\begin{array}{ccc}\omega^{a} & & \\
1 & \omega^{a} & \\
& 1 & \omega^{a}\end{array}\right)$ & $0 \leq a \leq 2$ & 3 \\
\hline$A_{4}^{(a)}$ & $\left(\begin{array}{lll}\pi^{a} & & \\
& \pi^{a} & \\
& & \pi^{-2 a}\end{array}\right)$ & $\begin{array}{l}0 \leq a \leq 3 q+2 \\
a \not \equiv 0(\bmod q+1)\end{array}$ & $3 q$ \\
\hline$A_{\mathrm{s}}^{(a, i)}$ & $\left(\begin{array}{lll}\pi^{a} & & \\
\pi^{i} & \pi^{a} & \\
& & \pi^{-2 a}\end{array}\right)$ & $\begin{array}{l}0 \leq a \leq 3 q+2 \\
0 \leq i \leq 2 \\
i \equiv a(\bmod 3) \\
a \not \equiv 0(\bmod q+1)\end{array}$ & $3 q$ \\
\hline$A_{6}^{(a, b, c, i)}$ & $\left(\begin{array}{lll}\pi^{(3 a+i)} & & \\
& \pi^{(3 b+i)} & \\
& & \pi^{(3 c+i)}\end{array}\right)$ & $\begin{array}{l}1 \leq a, b, c \leq q+1 \\
0 \leq i \leq 2 \\
a, b, c \text { distinct }(\bmod q+1) \\
a+b+c+i \equiv 0(\bmod q+1) \\
\left(A^{(a, b, c)}=A^{(b, c, a)}\right. \\
\left.=A^{(b, a, c)} \text { etc. }\right)\end{array}$ & $q(q-1) / 2$ \\
\hline$A_{7}^{(b, i)}$ & $\left(\begin{array}{lll}\rho^{b-i} \pi^{i} & & \\
& \sigma^{b} \pi^{i} & \\
& & \sigma^{-b q} \pi^{i}\end{array}\right)$ & $\begin{array}{l}1 \leq b \leq q^{2}-1 \\
0 \leq i \leq 2 \\
b \not \equiv 0(\bmod q-1) \\
\left(A^{(-b q, i)}=A^{(b, i)}\right)\end{array}$ & $\begin{array}{l}3(q+1) \\
\cdot(q-2) / 2\end{array}$ \\
\hline$A_{8}^{(a, i)}$ & $\left(\begin{array}{lll}\gamma^{a} \pi^{i} & & \\
& \gamma^{-a q} \pi^{i} & \\
& & \\
& & \gamma^{a q^{2}} \pi^{i}\end{array}\right)$ & $\begin{array}{l}1 \leq a \leq q^{3}+1 \\
0 \leq i \leq 2 \\
a \neq 0\left(\bmod q^{2}-q+1\right) \\
a \equiv-i(\bmod q+1) \\
\left(A_{8}^{(a, i)}=A_{8}^{(-a q, i)}=A_{8}^{\left(a q^{2}, i\right)}\right)\end{array}$ & $q(q-1)$ \\
\hline
\end{tabular}

If $\left\{\rho^{b-i} \pi^{i}, \sigma^{b} \pi^{i}, \sigma^{-b q} \pi^{i}\right\}=\left\{\rho^{b^{\prime}-i^{\prime}} \pi^{i^{\prime}}, \sigma^{b^{\prime}} \pi^{i^{\prime}}, \sigma^{-b^{\prime} q} \pi^{i^{\prime}}\right\}$ for some $b, b^{\prime}, i$, $i^{\prime}$ as in Step 2, then $\rho^{b-i} \pi^{i}=\rho^{b^{\prime}-i^{\prime}} \pi^{i^{\prime}}$ forces $i=i^{\prime}$ and $b \equiv b^{\prime}(\bmod q+1)$. Now $\left\{\sigma^{b}, \sigma^{-b q}\right\}=\left\{\sigma^{b^{\prime}}, \sigma^{-b^{\prime} q}\right\}$ so $\{b,-b q\} \equiv\left\{b^{\prime},-b^{\prime} q\right\}\left(\bmod q^{2}-1\right)$. 
If $\left\{\gamma^{a} \pi^{i}, \gamma^{-a q} \pi^{i}, \gamma^{a q^{2}} \pi^{i}\right\}=\left\{\rho^{b-j} \pi^{j}, \sigma^{b} \pi^{j}, \sigma^{-b q} \pi^{j}\right\}$ for some $a, i, b, j$ as in Step 2, then we may assume $\gamma^{a} \pi^{i}=\rho^{b-j} \pi^{j} \in\langle\pi\rangle$. So $\left\{\gamma^{a} \pi^{i}, \gamma^{-a q} \pi^{i}\right.$, $\left.\gamma^{a q^{2}} \pi^{i}\right\}=\left\{1, \omega, \omega^{2}\right\}=\left\{\pi^{q+1}, \pi^{2(q+1)}, \pi^{3(q+1)}\right\}$ by Substep 3.1. But no set $\left\{\rho^{b-i} \pi^{i}, \sigma^{b} \pi^{i}, \sigma^{-b q} \pi^{i}\right\}$ has this form, as shown a few lines above. The contradiction completes the proof.

Step 4. $s_{r}(H)=p^{r}=s_{r}\left(N_{H}(P)\right)+1$.

Proof. $s_{r}(H)=p^{r}$ by Lemma 4.2. From [14, p. 242], we may assume that $N:=N_{H}(P)=P\langle g\rangle$, where

$$
g=\left(\begin{array}{lll}
\sigma & & \\
& \sigma^{-q} & \\
& & \rho
\end{array}\right)
$$

has order $q^{2}-1$. Now $N$ has $q^{2}-1$ conjugacy classes of semisimple elements, with representatives $g^{j}, 1 \leq j \leq q^{2}-1$. So $\tau^{r}$ fixes the $N$-class of $g^{j}$ if and only if $\left(g^{j}\right)^{p^{r}}=g^{j}$, which occurs if and only if $\operatorname{order}\left(g^{j}\right) \mid\left(p^{r}-1, q^{2}-1\right)=$ $p^{r}-1$. Thus $\tau^{r}$ fixes $p^{r}-1$ semisimple conjugacy classes of $N$.

Step 5. $s_{r}(E)=p^{r}$ if $r \mid k ; s_{r}(E)=p^{r}+2$ if $r \nmid k ; s_{r}\left(N_{E}(P)\right)=p^{r}-1$.

Proof. Since

$$
E=H\langle x\rangle, \quad \text { where } x=\left(\begin{array}{lll}
\pi^{-1} & & \\
& \pi^{-1} & \\
& & \pi^{2}
\end{array}\right),
$$

we have $E \leq \mathrm{SU}_{3}\left(q^{6}\right)=\mathrm{SU}_{3}\left(\left(p^{3 k}\right)^{2}\right)$.

Except for three classes, say $A, C$ and $D$, of $E$ with eigenvalues $\{1, \omega$, $\left.\omega^{2}\right\}$, the semisimple conjugacy classes of $E$ remain distinct in $\mathrm{SU}_{3}\left(q^{6}\right)$ by Step 3. The semisimple classes of $H$ do not fuse in $E$ by Step 1. Now $E=$ $H \cup H x \cup H x^{2}$, each coset is a union of conjugacy classes and $H x^{2}=(H x)^{-1}$. So we may assume (by Step 1) that $A \subseteq H, C \subseteq H x$ and $D=C^{-1}$. By Step 4, $\tau^{r}$ fixes $p^{r}$ semisimple classes of both $H$ and $\mathrm{SU}_{3}\left(q^{6}\right)$. Since $A^{\tau^{r}}=A$ and $\{C, D\}^{\tau^{r}}=\{C, D\}$, it follows that either $C^{\tau^{r}}=D$ and $s_{r}(E)=p^{r}$ or $C^{\tau^{r}}=C$ and $s_{r}(E)=p^{r}+2$. Now $x^{\tau^{r}}=x^{p^{r}}$, so $C^{\tau^{r^{r}}}=C$ if and only if $H x^{p^{r}}=H x$, which is equivalent to $p^{r} \equiv 1(\bmod 3)$. Since $3 \mid q+1$ means that $p \equiv-1$ $(\bmod 3)$ and $k$ is odd, we see that $s_{r}(E)=p^{r}$ if and only if $r \mid k$.

We may assume that

$$
N_{E}(P)=P\langle g x\rangle, \quad \text { where } g x=\left(\begin{array}{lll}
\sigma \pi^{-1} & & \\
& \sigma^{-q} \pi^{-1} & \\
& & \rho \pi^{2}
\end{array}\right) .
$$

Then $g x$ has order $3\left(q^{2}-1\right)$, and the elements $(g x)^{j}, 0 \leq j<3\left(q^{2}-1\right)$, are representatives for the semisimple classes of $N_{E}(P)$. Since $(g x)^{\tau^{r}}=(g x)^{p^{r}}$, we see that the class of $(g x)^{j}$ is fixed by $\tau^{r}$ if and only if $(g x)^{j\left(p^{r}-1\right)}=1$. 
Since $p^{r}-1 \mid 3\left(q^{2}-1\right)$, it is clear that $\tau^{r}$ fixes exactly $p^{r}-1$ semisimple classes of $N_{E}(P)$.

Step 6. If $r \mid k$ then $s_{r}(Q / Z(Q))=s_{r}(Q)$ and $s_{r}\left(N_{Q}(P) / Z(Q)\right)=s_{r}\left(N_{Q}(P)\right)$ for $Q=H$ and for $Q=E$.

Proof. It is implicit here that $d=3$, so $Z(E)=Z(H)=\langle\omega I\rangle$. Let $Q$ denote either $H$ or $E$, and let $X$ equal either $Q$ or $N_{Q}(P)$. If $r \mid k$ then $r$ odd and $p \equiv-1 \quad(\bmod 3)$ imply that $(\omega I)^{\tau^{r}}=\omega^{p^{r}} I=(\omega I)^{-1}$. Thus if $\psi$ is any irreducible Brauer character of $X$ with $Z(Q) \not \operatorname{ker} \psi$, then $\left(\psi_{Z(Q)}\right)^{\tau^{r}} \neq \psi_{Z(Q)}$, and hence $\psi^{\tau^{r}} \neq \psi$. So the number of irreducible Brauer characters of $X$ fixed by $\tau^{r}$ equals the number of irreducible Brauer characters of $X / Z(Q)$ fixed by $\tau^{r}$. Step 6 now follows by Lemma 3.6 of Feit [11, p. 146], and Brauer's permutation lemma.

Step 7. If $r \nmid k$ and $3 \nmid \frac{2 k}{r}$ then

$$
s_{r}(H / Z(H))=\left(p^{r}-1\right) / 3+1
$$

and

$$
s_{r}\left(N_{H}(P) / Z(H)\right)=\left(p^{r}-1\right) / 3 \text {. }
$$

Proof. Let $X$ denote either $H$ or $N_{H}(P)$. Now $r$ is even, so $\omega^{p^{r}}=\omega$. If $C$ is a semisimple class of $X$ such that $C^{\tau^{r}}=\left(\omega^{ \pm 1}\right) C$, then $C^{\tau^{3 r}}=C$. It follows that $3 \mid \operatorname{order}\left(\left.\tau^{r}\right|_{X}\right)$. But $\operatorname{order}\left(\left.\tau^{r}\right|_{H}\right)=2 k / r$, which contradicts our hypotheses. Hence, $\tau^{r}$ fixes only the images in $X / Z(H)$ of those semisimple classes of $X$ which are already fixed by $\tau^{r}$. The preimages of a class of $X / Z(H)$ are of the form $C, \omega C, \omega^{2} C$, all distinct unless $X=H$ and $C$ contains

$$
\left(\begin{array}{lll}
1 & & \\
& \omega & \\
& & \omega^{2}
\end{array}\right) \text {. }
$$

The result now follows from Step 4.

Step 8. If $r \nmid k$ and $3 \mid 2 k / r$ then $s_{r}(H / Z(H))=p^{r}$ and $s_{r}\left(N_{H}(P) / Z(H)\right)=$ $p^{r}-1$.

Proof. Since $p \equiv-1 \quad(\bmod 3), k$ and $r / 2$ are odd and $r$ is even, we have that

$$
3 \mid 1-p^{r / 2}+p^{2 r / 2}-\cdots+p^{(2 k / r-1) r / 2}=\left(p^{k}+1\right) /\left(p^{r / 2}+1\right) .
$$

Let $n=\left(p^{k}+1\right) /\left(3\left(p^{r / 2}+1\right)\right)$. Let

$$
s=\left(\begin{array}{ccc}
\rho^{-n} & & \\
& \rho^{-n} & \\
& & \rho^{2 n}
\end{array}\right) \in H .
$$

Then $s^{3} \in \mathrm{SU}_{3}\left(p^{r}\right)=\mathrm{SU}_{3}\left(\left(p^{r / 2}\right)^{2}\right) \subseteq H$, as $\left(\rho^{n}\right)^{3}$ is a " $\rho$ " (of order $\left.p^{r / 2}+1\right)$ for $\mathrm{SU}_{3}\left(p^{r}\right)$. Note that $\mathrm{SU}_{3}\left(p^{r}\right)\langle s\rangle$ equals $E\left(p^{r}\right)$ and is stabilized by $\tau^{r}$. Now 
$\rho^{-n p^{r}} \rho^{n}=\rho^{\left(1-p^{r / 2}\right)(q+1) / 3}$ implies that $s^{\tau^{r}} s^{-1}=\omega^{2} I$. Hence for all $h \in \mathrm{SU}_{3}\left(p^{r}\right)$ and $i=1,2, h^{\tau^{r}}=h$ and $\left(h s^{i}\right)^{\tau^{r}}=\omega^{2 i} h s^{i}$. So for every semisimple conjugacy class $C$ of $E\left(p^{r}\right), C^{\tau^{r}}=\omega^{j} C$ for some $j$. Since only the three classes of $E\left(p^{r}\right)$ with eigenvalues $\left\{1, \omega, \omega^{2}\right\}$ fuse in $H$, by Step 3 , and $E\left(p^{r}\right)$ has $3 p^{r}$ semisimple classes, by Step 2, we have found $3 p^{r}-2$ semisimple classes of $H$ which are fixed modulo $Z(H)$ by $\tau^{r}$. So $\tau^{r}$ fixes at least $\left(3 p^{r}-3\right) / 3+1=$ $p^{r}$ semisimple classes, and hence at least $p^{r}$ irreducible Brauer characters, of $H / Z(H)$. But $\tau^{r}$ fixes only $p^{r}$ Brauer characters of $H$ (by Step 4), and so $\tau^{r}$ fixes exactly $p^{r}$ semisimple classes of $H / Z(H)$.

Recall from Step 4 that $N_{H}(P)=P\langle g\rangle$, where

$$
g=\left(\begin{array}{lll}
\sigma & & \\
& \sigma^{-q} & \\
& & \rho
\end{array}\right)
$$

has order $q^{2}-1$. Now $\tau^{r}$ fixes the class of $g^{j}(\bmod Z(H))$ if and only if $\left(g^{j}\right)^{p^{r}}=\omega^{t} g^{j}$ for some $t$, which is equivalent to $\sigma^{3 j\left(p^{r}-1\right)}=1$. Since $3\left(p^{r}-1\right) \mid q^{2}-1$ (as $r$ is even and $\left.3 \mid 2 k / r\right), j$ is a solution if and only if $\left(q^{2}-1\right) / 3\left(p^{r}-1\right) \mid j$, and there are $3\left(p^{r}-1\right)$ such $j\left(\bmod q^{2}-1\right)$. Hence $\tau^{r}$ fixes $p^{r}-1$ semisimple classes of $N_{H}(P) / Z(H)$.

Step 9. If $r \nmid k$ then $s_{r}(E / Z(E))=p^{r}+2$ and $s_{r}\left(N_{E}(P) / Z(E)\right)=p^{r}-1$.

Proof. Let $m=(q+1) /\left(p^{r / 2}+1\right)$ (an integer, as noted in Step 8). Let

$$
u=x^{m}=\left(\begin{array}{lll}
\pi^{-m} & & \\
& \pi^{-m} & \\
& & \pi^{2 m}
\end{array}\right) .
$$

So

$$
u^{3}=\left(\begin{array}{lll}
\rho^{-m} & & \\
& \rho^{-m} & \\
& & \rho^{2 m}
\end{array}\right),
$$

where $\rho^{m}$, of order $p^{r / 2}+1$, is a " $\rho$ " for $\mathrm{SU}_{3}\left(\left(p^{r / 2}\right)^{2}\right)=\mathrm{SU}_{3}\left(p^{r}\right) \leq H$. Thus $\mathrm{SU}_{3}\left(p^{r}\right)\langle u\rangle=E\left(p^{r}\right) \leq E\left(q^{2}\right)$, and $E\left(p^{r}\right)$ is stable under $\tau^{r}$. Since $\tau^{r}$ is trivial on $\mathrm{SU}_{3}\left(p^{r}\right)$, and since $\pi^{-m p^{r}} \pi^{m}=\pi^{\left(1-p^{r / 2}\right)(q+1)}$ implies that $u^{\tau^{r}} u^{-1} \in\langle\omega I\rangle$, it follows that $\tau^{r}$ fixes every semisimple conjugacy class of $E\left(p^{r}\right)$ (modulo $Z(E))$. Since the semisimple classes of $E\left(p^{r}\right)$ do not fuse in $E\left(q^{2}\right)$, by Steps 2 and 3, we have that $\tau^{r}$ fixes at least $3 p^{r}$ semisimple classes of $E\left(q^{2}\right)$ (modulo $\left.Z\left(E\left(q^{2}\right)\right)\right)$. Since the three classes of $E\left(q^{2}\right)$ with eigenvalues $1, \omega, \omega^{2}$ are not equal $(\bmod Z(E))$, we see that $\tau^{r}$ fixes at least $\left[\left(3 p^{r}-3\right) / 3\right]+3=p^{r}+2$ semisimple classes, and hence irreducible Brauer characters, of $E / Z(E)$. Step 5 implies that $\tau^{r}$ fixes at most $p^{r}+2$ irreducible Brauer characters of $E / Z(E)$, and so $s_{r}(E / Z(E))=p^{r}+2$ in this case. 
As before,

$$
N_{E}(P)=P\langle g x\rangle, \quad \text { where } g x=\left(\begin{array}{lll}
\sigma \pi^{-1} & & \\
& \sigma^{-q} \pi^{-1} & \\
& & \rho \pi^{2}
\end{array}\right),
$$

and the matrices $(g x)^{j}, 0 \leq j<3\left(q^{2}-1\right)$, represent the semisimple conjugacy classes of $N_{E}(P)$. Now $(g x)^{j \tau^{r}} \equiv(g x)^{j} \quad(\bmod Z(E))$ if and only if $(g x)^{3 j\left(p^{r}-1\right)}=1$. Since $3\left(p^{r}-1\right) \mid 3\left(q^{2}-1\right)$, there are $3\left(p^{r}-1\right)$ solutions for $j$ and $s_{r}\left(N_{E}(P) / Z(E)\right)=p^{r}-1$.

Step 10. The number of $p$-singular classes of $H$ (hence of $N_{H}(P)$, since $P$ is a T.I. set in $H$ ) fixed by $\tau^{r}$ is $1+(2, p-1)$ if $r \mid k$, or $p^{r / 2}+d^{2}+1$ if $r \nmid k$. Proof. Denote the classes $C_{2}^{(j)}, C_{5}^{(j)}$ of Step 1 together as $C^{(j)}$, with canonical representative

$$
\left(\begin{array}{ccc}
\rho^{j} & & \\
1 & \rho^{j} & \\
& & \rho^{j}
\end{array}\right)
$$

for $1 \leq j \leq q+1$. Then $C^{(j) \tau^{r}}=C^{(j)}$ if and only if $\rho^{j\left(p^{r}-1\right)}=1$. Since $\left(q+1, p^{r}-1\right)$ equals $(2, p-1)$ if $r \mid k, p^{r / 2}+1$ if $r+k$, there are, respectively, $(2, p-1)$ or $p^{r / 2}+1$ solutions for $j$.

If $d=1$ then there is only one class $C_{3}^{(j, l)}$ and it is fixed by $\tau^{r}$. If $d=3$ (so that $p \equiv 2(\bmod 3)$ and $k$ is odd), then $\tau^{r}$ fixes $C_{3}^{(j, l)}$ if and only if $\theta^{l p^{r}-l} \in\left\langle\theta^{3}\right\rangle$ and $\omega^{j\left(p^{r}-1\right)}=1$. But these conditions together are equivalent to either $p^{r}-1 \equiv 0(\bmod 3)$ (which occurs just when $r$ is even, i.e. $r \nmid k$ ) or $l=j=0$. The result follows.

Step 11. Let $d=3$. The number of $p$-singular classes of $H / Z(H)$ (hence of $\left.N_{H}(P) / Z(H)\right)$ fixed by $\tau^{r}$ equals $1+(2, p-1)$ if $r \mid k,\left(p^{r / 2}+1\right) / 3+3$ if $r \nmid k$ and $3+2 k / r$, or $p^{r / 2}+4$ if $r+k$ and $3 \mid 2 k / r$.

Proof. There are three distinct images of the classes $C_{3}^{(j, l)}$ in $H / Z(H)$, namely the images of $C_{3}^{(0, l)}, l=0,1,2$. But $C_{3}^{(0, l)}$ is fixed by $\tau^{r}$ if and only if $\theta^{l\left(p^{r}-1\right)} \in\left\langle\theta^{3}\right\rangle$. So only one such class in $H / Z(H)\left(C_{3}^{(0,0)}\right)$ is fixed if $r \mid k$, but all three are fixed if $r \nmid k$.

The image of $C^{(j)}$ (as in Step 10) in $H / Z(H)$ is fixed by $\tau^{r}$ if and only if $\rho^{j\left(p^{r}-1\right)} \in\langle\omega\rangle$, which is equivalent to $(q+1) / 3 \mid j\left(p^{r}-1\right)$. The number of solutions $j$ with $1 \leq j \leq q+1$ is $3\left((q+1) / 3, p^{r}-1\right)$, which yields $\left((q+1) / 3, p^{r}-1\right)$ distinct images in $H / Z(H)$ fixed by $\tau^{r}$. Since

$$
\left(\frac{q+1}{3}, p^{r}-1\right)= \begin{cases}(2, p-1) & \text { if } r \mid k, \\ \left(p^{r / 2}+1\right) / 3 & \text { if } r \nmid k \text { and } 3 \nmid \frac{2 k}{r}, \\ p^{r / 2}+1 & \text { if } r \nmid k \text { and } 3 \mid \frac{2 k}{r},\end{cases}
$$

the result holds. 
Step 12. The number of $p$-singular classes of $E$ (hence of $\left.N_{E}(P)\right)$ fixed by $\tau^{r}$ equals $1+(2, p-1)$ if $r \mid k$, or $p^{r / 2}+4$ if $r \nmid k$.

Proof. Denote the classes $A_{2}^{(a)}, A_{5}^{(a)}$ of Step 2 together as $A^{(a, i)}$ with representative

$$
\left(\begin{array}{lll}
\pi^{a} & & \\
\pi^{i} & \pi^{a} & \\
& & \pi^{-2 a}
\end{array}\right)
$$

for $0 \leq a \leq 3 q+2,0 \leq i \leq 2, i \equiv a(\bmod 3)$. Then $\tau^{r}$ fixes $A^{(a, i)}$ if and only if $\pi^{a\left(p^{r}-1\right)}=1$. There are $\left(3(q+1), p^{r}-1\right)$ solutions $a$, i.e. $p^{r / 2}+1$ if $r \nmid k$ and $(2, p-1)$ if $r \mid k$. The class $A_{3}^{(a)}, 0 \leq a \leq 2$, is fixed by $\tau^{r}$ if and only if $\omega^{a\left(p^{r}-1\right)}=1$, and there are $\left(3, p^{r}-1\right)$ solutions $a$. The step is proved.

Step 13. The number of $p$-singular classes of $E / Z(E)$ (hence of $N_{E}(P) / Z(E)$ ) fixed by $\tau^{r}$ equals $1+(2, p-1)$ if $r \mid k$, or $p^{r / 2}+2$ if $r+k$.

Proof. The $3 q+3$ classes $A^{(a, i)}$ as in Step 12 have $q+1$ distinct images in $E / Z(E)$, and the image of $A^{(a, i)}$ is fixed by $\tau^{r}$ if and only if $\pi^{a\left(p^{r}-1\right)} \in\langle\omega\rangle$. Since $\pi^{3}=\rho$, this is equivalent to $\rho^{a\left(p^{r}-1\right)}=1$. There are $3\left(q+1, p^{r}-1\right)$ solutions for $0 \leq a \leq 3 q+2$, hence $\left(q+1, p^{r}-1\right)$ images in $E / Z(E)$ fixed by $\tau^{r}$. The classes $A_{3}^{(a)}$ have but one image in $E / Z(E)$, which is clearly fixed by $\tau^{r}$.

All values of $s_{r}$ and $c_{r}$ in Proposition 4.4 have been established by Steps $1-13$.

Step 14. $h z_{r}(H)=p^{r}+1$ if $r \mid k$, or $p^{r}+d^{2}$ if $r+k ; h z_{r}(H / Z(H))=p^{r}+1$ if $r \mid k, p^{r}+3$ if $r \nmid k$ and $3 \mid(2 k / r),\left(p^{r}-1\right) / 3+4$ if $r+k$ and $3 \nmid 2 k / r$.

Proof. According to [21], $H$ has $q+1$ characters of raised height: one character $\chi_{q(q-1)}$ of degree $q(q-1)$, which is clearly fixed by $\tau^{r}$, and $q$ characters of degree $q\left(q^{2}-q+1\right)$, which we denote here as $\chi^{(u)}, 1 \leq u \leq q$, such that the value of $\chi^{(u)}$ on any $g_{j} \in C_{5}^{(j)}$ is $-\varepsilon^{u j}$, where $\varepsilon$ is a complex primitive $(q+1)$ st root of unity. Then

$$
\chi^{(u)\left(\tau^{r}\right)^{-1}}\left(g_{j}\right)=\chi^{(u)}\left(g_{j p^{r}}\right)=-\varepsilon^{u j p^{r}},
$$

where we read $j p^{r} \bmod q+1$. So $\chi^{(u) \tau^{r}}=\chi^{(u)}$ if and only if $\varepsilon^{u p^{r}}=\varepsilon^{u}$, i.e. $\varepsilon^{u\left(p^{r}-1\right)}=1$. The number of solutions $u$ is $\left(q+1, p^{r}-1\right)-1$, i.e. $(2, p-1)-1$ if $r \mid k$ or $p^{r / 2}$ if $r \nmid k$. Hence $\tau^{r}$ fixes either $(2, p-1)$ (if $r \mid k$ ) or $p^{r / 2}+1$ (if $r \nmid k$ ) characters of $H$ of raised height.

If $d=3$ then $Z(H) \leq \operatorname{ker} \chi_{q(q-1)}$, and $Z(H) \leq \operatorname{ker} \chi^{(u)}$ if and only if $u \equiv 0(\bmod 3) \quad\left(\right.$ by $\left[21\right.$, p. 488]). The number of solutions to $\varepsilon^{u\left(p^{r}-1\right)}=1$ for $1 \leq u \leq q$ and $u \equiv 0(\bmod 3)$ is $(2, p-1)-1$ if $r \mid k, p^{r / 2}$ if $r \nmid k$ 
and $3 \mid 2 k / r$, or $\left(p^{r / 2}+1\right) / 3-1$ if $r \nmid k$ and $3 \nmid 2 k / r$. So $\tau^{r}$ fixes either $(2, p-1)$ (if $r \mid k), p^{r / 2}+1$ (if $r \nmid k$ and $3 \mid 2 k / r$ ), or $\left(p^{r / 2}+1\right) / 3$ (if $r \nmid k$ and $3 \nmid 2 k / r)$ irreducible characters of $H / Z(H)$ of raised height. Since $\tau^{r}$ fixes $c_{r}(h)$ irreducible characters of $H$ (by Brauer's permutation lemma), and $c_{r}(H / Z(H))$ irreducible characters of $H / Z(H)$, the result follows from the now established values of $c_{r}(H)$ and $c_{r}(H / Z(H))$.

Step 15. $h z_{r}\left(N_{H}(P)\right)=p^{r}$ if $r \mid k$, or $p^{r}+d^{2}-1$ if $r \nmid k ; h z_{r}\left(N_{H}(P) / Z(H)\right)=$ $p^{r}$ if $r \mid k, p^{r}+2$ if $r \nmid k$ and $3 \mid 2 k / r$, or $\left(p^{r}-1\right) / 3+3$ if $r \nmid k$ and $3 \nmid 2 k / r$.

Proof. The irreducible characters of $N:=N_{H}(P)$ with height zero are clearly the characters of $N / P^{\prime}$, where $N / P^{\prime}$ has the structure of an elementary abelian group $\left(P / P^{\prime}\right)$ of order $q^{2}$, extended by a cyclic group of order $q^{2}-1$, which induces a fixed-point-free action of order $\left(q^{2}-1\right) / d$ by conjugation on $P / P^{\prime}$ $\left[14\right.$, p. 242]. Now $N / P^{\prime}$ has $q^{2}-1$ (linear) characters with $P / P^{\prime}$ in their kernel, and $p^{r}-1$ of them are fixed by $\tau^{r}$.

If $d=1, N / P^{\prime}$ has but one irreducible character $\zeta$ with $P / P^{\prime} \not k \operatorname{ker} \zeta$ and hence $h z_{r}(N)=p^{r}$. If $d=3$ then there are nine characters of $N / P^{\prime}$ with $P$ not in their kernels, and they vanish off $P \times Z(H)$. So they are uniquely determined by their values on the nine classes $C_{3}^{(j, l)}, 0 \leq j, l \leq 2$. Thus $\tau^{r}$ fixes as many of these characters as it does the $C_{3}^{(j, l)}$. This number is nine if $r \nmid k$ or one if $r \mid k$ (as in Step 10). The result for $h z_{r}(N)$ follows.

When $d=3$, there are $\left(q^{2}-1\right) / 3$ linear characters of $N / P^{\prime} Z(H)$ with $P$ in their kernels. It is easily seen that $p^{r}-1$ of them are fixed by $\tau^{r}$ if $r \mid k$ or if $r \nmid k$ and $3 \mid 2 k / r$, but only $\left(p^{r}-1\right) / 3$ are fixed if $r \nmid k$ and $3 \nmid 2 k / r$. There are three characters of $N / P^{\prime} Z(H)$ with $P$ not in their kernels, and they vanish off the images of the three classes $C_{3}^{(0, l)}$ of $N$. So $\tau^{r}$ fixes as many such characters as it does the $C_{3}^{(0, l)}$, i.e. it fixes one if $r \mid k$ and three if $r \nmid k$.

Step 16. $h z_{r}(E)=p^{r}+1$ if $r \mid k$ or $p^{r}+5$ if $r+k ; h z_{r}(E / Z(E))=p^{r}+1$ if $r \mid k$ or $p^{r}+3$ if $r+k$.

Proof. Let $\varepsilon$ again be a complex $(q+1)$ st root of unity, and let $\mu$ be a cube root of $\varepsilon$. Recall that

$$
x=\left(\begin{array}{lll}
\pi^{-1} & & \\
& \pi^{-1} & \\
& & \pi^{2}
\end{array}\right),
$$

and let $\nu$ be the linear character of $\langle x\rangle$ such that $\nu\left(x^{-j}\right)=\mu^{j}$. It follows from the character table of $J=G U_{3}\left(q^{2}\right)[10]$ and the fact that $J\langle\pi I\rangle=E\langle\pi I\rangle$ that $E$ has $3 q$ characters $\chi^{(u)}$ of degree $q\left(q^{2}-q+1\right)$, for $1 \leq u \leq 3 q+3$ with $u \neq \equiv 0(\bmod q+1)$, such that

$$
\begin{aligned}
& \chi^{(u)}\left(x^{-j}\right)=(q-1) \mu^{u j}+q \mu^{-2 u j} \quad \text { if } q+1 \nmid j, \\
& \chi^{(u)}(\omega I)=q\left(q^{2}-q+1\right) \mu^{u(q+1)} .
\end{aligned}
$$


Thus

$$
\left.\chi^{(u)}\right|_{\langle x\rangle}=(q-1) \nu^{u}+q \nu^{-2 u}+(q-1)^{2} \nu^{u} R(\langle x\rangle /\langle\omega I\rangle),
$$

where $R$ denotes the regular representation. So $\chi^{(u)^{r^{r}}}=\chi^{(u)}$ if and only if $\mu^{u\left(p^{r}-1\right)}=1$. Hence the number of fixed $\chi^{(u)}$ is $(2, p-1)-1$ if $r \mid k$, and $p^{r / 2}-2$ if $r \nmid k$. Since a given $\chi^{(u)}$ has $Z(E)$ in its kernel if and only if $3 \mid u$, we see that all of the $\chi^{(u)}$ fixed by $\tau^{r}$ have $Z(E)$ in their kernel.

Again, the character table of $J$ and the fact that $J\langle\pi I\rangle=E\langle\pi I\rangle$ yield that $E$ has three characters $\zeta^{(i)}$ of degree $q^{2}-q$, for $0 \leq i \leq 2$, such that

$$
\zeta^{(i)}\left(x^{-j}\right)=-(q-1) \mu^{i j(q+1)}
$$

for all $j$ with $q+1 \nmid j$ and $i=0,1,2$. So $\tau^{r}$ fixes $\zeta^{(i)}$ if and only if $\mu^{i\left(p^{r}-1\right)(q+1)}=1$. Hence all three are fixed if $r \nmid k$ but only $\zeta^{(0)}$ is if $r \mid k$. All three of the $\zeta^{(i)}$ have $Z(E)$ in their kernel.

The $\chi^{(u)}$ and $\zeta^{(i)}$ comprise all of the characters of $E$ of raised height, and our results show that $\tau^{r}$ fixes exactly $(2, p-1)$ such characters (of $E$ and of $E / Z(E))$ if $r \mid k$, while $p^{r / 2}+1$ are fixed if $r \nmid k$. Step 16 follows from the established values of $c_{r}(E)$ and $c_{r}(E / Z(E))$.

Step 17. $h z_{r}\left(N_{E}(P)\right)=p^{r}$ if $r \mid k, p^{r}+2$ if $r \nmid k ; h z_{r}\left(N_{E}(P) / Z(E)\right)=p^{r}$.

Proof. The height zero characters of $N_{E}(P)$ are exactly those with $P^{\prime}$ in their kernel. $\quad N_{E}(P) / P^{\prime}$ is the semidirect product of the elementary abelian group $P / P^{\prime}$ of order $q^{2}$ with a cyclic group of order $3\left(q^{2}-1\right)$ which has a fixedpoint-free action of order $q^{2}-1$ on $P / P^{\prime}$. So $N_{E}(P)$ has $3\left(q^{2}-1\right)$ (linear) characters with $P$ in their kernels, and $p^{r}-1$ of them are fixed by $\tau^{r}$. Each such fixed character has $Z(E)$ in its kernel.

Now $N_{E}(P) / P^{\prime}$ has only three characters with kernel not containing $P$, and they vanish off $P \times Z(E)$. So they are determined by their values on the classes $A_{3}^{(a)}, 0 \leq a \leq 2$. Hence $\tau^{r}$ fixes all three characters when $r \nmid k$, and only the unique such character with $Z(E)$ in its kernel when $r \mid k$. This completes the proof of Step 17.

Step 18. $d z_{r}(H)=1=d z_{r}(H / Z(H)) ; d z_{r}(E)=d z_{r}(E / Z(E))=1$ if $r \mid k$, or 3 if $r \nmid k$.

Proof. $H$ has a unique $p$-block of defect zero [21], so its character, which has $Z(H)$ in its kernel, is fixed by $\tau^{r}$. Since $J\langle\pi I\rangle=E\langle\pi I\rangle$, the character table of $J$ yields three blocks of defect zero for $E$, with character $\chi_{i}$ (of degree $q^{3}$ ) for $0 \leq i \leq 2$ such that

$$
\chi_{i}\left(x^{-j}\right)=q \mu^{i j(q+1)}
$$

for all $j$ with $q+1 \nmid j$. Each $\chi_{i}$ has $Z(E)$ in its kernel. Now $\tau^{r}$ fixes $\chi_{i}$ if and only if $\mu^{i\left(p^{r}-1\right)(q+1)}=1$. Hence $d z_{r}(E)=d z_{r}(E / Z(E))$ is as claimed.

Step 19. Conclusion. 
Since $N_{H}(P)$ and $N_{E}(P)$ can have no $p$-blocks of defect zero, all entries of the table have been verified and the proof of Proposition 4.3 is complete.

Proof of Proposition 4.5. (a) This is immediate, since $\delta$ fixes every semisimple class of $H$ and of $N$.

(b) By Step 1 of the proof of Proposition 4.4, $\delta$ fixes every $p$-singular class of $H$ (and so of $N$ ) except for the $C_{3}^{(j, l)}$. Here, for each fixed $j, \delta$ cycles the three classes $C_{3}^{(j, l)}, 0 \leq l \leq 2$. If $r \nmid k, \tau^{r}$ fixes each $C_{3}^{(j, l)}$. Hence $\delta \tau^{r}$ fixes none of them and so $c_{r}^{\delta}(H)=c_{r}(H)-9, c_{r}^{\delta}(N)=c_{r}(N)-9$. If $r \mid k, \tau^{r}$ permutes $\omega$ and $\omega^{2}$, and fixes $C_{3}^{(j, l)}$ only when $j=l=0$. So $\delta \tau^{r}$ fixes just one $C_{3}^{(j, l)}$, when $j=0$ and $l=1$ or 2. So $c_{r}^{\delta}(H)=c_{r}(H)$ and $c_{r}^{\delta}(N)=c_{r}(N)$. The result follows from $c_{r}(N)+1=c_{r}(H)$ in Proposition 4.4.

(c) Again, $\delta$ fixes all classes of $H / Z(H)$, and of $N / Z(H)$, except for the three images of $C_{3}^{(0, l)}$ which it cycles. Since $\tau^{r}$ fixes each $C_{3}^{(0, l)}$ if $r \nmid k$, and fixes only $C_{3}^{(0,0)}$ when $r \mid k$, the result follows as in part (b).

(d) The character table for $H$ [21] shows that the characters of raised height vanish on the classes $C_{3}^{(j, l)}$ which are the only classes not fixed by $\delta$. So $\delta$ fixes all characters of raised height. So if $m_{r}$ is the number of raised height characters fixed by $\tau^{r}, h z_{r}^{\delta}(H)=c_{r}^{\delta}(H)-m_{r}=c_{r}^{\delta}(H)-\left(c_{r}(H)-h z_{r}(H)\right)$, so that part (b) and Proposition 4.4 yield the desired values for $h z_{r}^{\delta}(H)$.

Since $\delta$ fixes each of the $q^{2}-1$ (linear) characters of $N / P, \delta \tau^{r}$ fixes $p^{r}-1$ such characters. The other nine characters of $N / P^{\prime}$ vanish off $P \times Z(H)$, hence the number of them fixed by $\delta \tau^{r}$ equals the number of $C_{3}^{(j, l)}$ fixed by $\delta \tau^{r}$. This number is one if $r \mid k$ and zero if $r \nmid k$, as in the proof of part (b). Thus $h z_{r}^{\delta}(N)$ equals $p^{r}$ if $r \mid k$, and $p^{r}-1$ otherwise.

(e) As in the proof of (d),

$$
h z_{r}^{\delta}(H / Z(H))=c_{r}^{\delta}(H / Z(H))-\left(c_{r}(H / Z(H))-h z_{r}(H / Z(H))\right),
$$

and the values for $h z_{r}^{\delta}(H / Z(H))$ follow from part (c) and Proposition 4.4.

If $r \mid k$ or if $r \nmid k$ but $3 \mid 2 k / r$, then each character of $N / P$ fixed by $\delta \tau^{r}$ has $Z(H)$ in its kernel. Otherwise only $\left(p^{r}-1\right) / 3$ of them have $Z(H)$ in their kernels.

If $r \mid k$ then $\tau^{r}$ fixes only $C_{3}^{(0,0)}$ among the $C_{3}^{(0, l)}$. Thus $\delta \tau^{r}$ fixes one nonlinear character of $N / P^{\prime} Z(H)$ and $h z_{r}^{\delta}(N / Z(H))=p^{r}$. If $r \nmid k$ then $\tau^{r}$ fixes all $C_{3}^{(0, l)}$ and so $\delta \tau^{r}$ fixes no nonlinear character of $N / P^{\prime} Z(H)$. Thus $h z_{r}^{\delta}(N / Z(H))$ equals $p^{r}-1$ if $3 \mid 2 k / r,\left(p^{r}-1\right) / 3$ if $3 \nmid 2 k / r$.

Proposition 4.6. Let $d=3, H=\mathrm{SU}_{3}\left(q^{2}\right), E=H\langle x\rangle$ as before, where

$$
x=\left(\begin{array}{lll}
\pi^{-1} & & \\
& \pi^{-1} & \\
& & \pi^{2}
\end{array}\right)
$$


and $\omega=\pi^{q+1}$. Let $r \mid 2 k$ but $r \nmid k$ and $3 \nmid 2 k / r$. Then the following assertions hold:

(a) There are $3 p^{r}$ semisimple classes of $E$ which are fixed modulo $Z(E)$ by $\tau^{r}$. Exactly $p^{r}$ of them are contained in $H, p^{r}$ of them are classes $C \subseteq H x^{2 k / r}$ with $C^{\tau^{r}}=\omega^{2} C$, and the other $p^{r}$ are classes $C \subseteq H x^{-2 k / r}$ with $C^{\tau^{r^{r}}}=\omega C$.

(b) There are $3 p^{r}-3$ semisimple classes of $N_{E}(P)$ which are fixed modulo $Z(E)$ by $\tau^{r}$. Exactly $p^{r}-1$ of them are in $N_{H}(P), p^{r}-1$ of them are classes $C \subseteq N_{H}(P) x^{2 k / r}$ with $C^{\tau^{r}}=\omega^{2} C$, and the other $p^{r}-1$ are classes $C \subseteq H x^{-2 k / r}$ with $C^{\tau^{r}}=\omega C$.

(c) There are $3 p^{r / 2}+6$-singular classes of $E$ which are fixed modulo $Z(E)$ by $\tau^{r}$. Exactly $p^{r / 2}+4$ of them are contained in $H, p^{r / 2}+1$ of them are classes $C \subseteq H x^{2 k / r}$ with $C^{\tau^{r}}=\omega^{2} C$, and the other $p^{r / 2}+1$ are classes $C \subseteq H x^{-2 k / r}$ with $C^{\tau^{r}}=\omega C$.

Proof. Let $y=x^{2 k / r}$. Since $3 \nmid 2 k / r$, we have $E=H \cup H y \cup H y^{-1}$. Since $r \nmid k$ implies that $p^{r} \equiv 1(\bmod 3), \tau^{r}$ stabilizes each coset of $H$ in $E$; each coset is a union of conjugacy classes. Let

$$
m=(q+1) /\left(p^{r / 2}+1\right)=1-p^{r / 2}+\cdots+p^{(2 k / r-1)) r / 2} \equiv \frac{2 k}{r} \quad(\bmod 3),
$$

and let $u=x^{m} \in H y$.

As in the proof of Step 9 of Proposition 4.4, $\mathrm{SU}_{3}\left(p^{r}\right)\langle u\rangle=E\left(p^{r}\right)$ and $\pi^{-m p^{r}} \pi^{m}=\pi^{\left(1-p^{r / 2}\right)(q+1)}$, hence $u^{p^{r}}=u^{\tau^{r}}=\pi^{\left(1-p^{r / 2}\right)(q+1)} u=\omega^{2} u$. Now $\mathrm{SU}_{3}\left(p^{r}\right) u \subseteq H u=H y$, and hence for all $h \in \mathrm{SU}_{3}\left(p^{r}\right),(h u)^{\tau^{r}}=\omega^{2} h u$. Thus if $C$ is any conjugacy class of $E\left(p^{r}\right)$ with $C \subseteq \mathrm{SU}_{3}\left(p^{r}\right) u, C^{\tau^{r}}=\omega^{2} C$. Then $C^{-1} \subseteq H u^{-1}$ and $C^{-1 \tau^{r}}=\omega C^{-1}$. There are $p^{r}$ semisimple conjugacy classes of $E\left(p^{r}\right)$ in $\mathrm{SU}_{3}\left(p^{r}\right) u$, and none fuse in $E$ by Step 3 of the proof of Proposition 4.4. So there are at least $p^{r}$ semisimple classes $C$ in $H y$ with $C^{\tau^{r}}=\omega^{2} C$. By Proposition 4.4, $\tau^{r}$ fixes $p^{r}$ semisimple classes of $H$ (which remain distinct in $E$ ), and fixes $3 p^{r}$ semisimple classes of $E$ modulo $Z(E)$. (The three classes of $E$ with eigenvalues $\left\{1, \omega, \omega^{2}\right\}$ are not multiples of any other class by an element of $Z(E)$, and are fixed by $\tau^{r}$.) Assertion (a) follows.

Now $N_{E}(P)=N_{H}(P) \cup N_{H}(P) y \cup N_{H}(P) y^{-1}$, and $N_{H}(P) u=N_{H}(P) y$. By Proposition 4.4, there are $p^{r}-1$ elements of the form $g^{j}$ in $N_{H}(P)$,

$$
g=\left(\begin{array}{lll}
\sigma & & \\
& \sigma^{-q} & \\
& & \rho
\end{array}\right) \text {, }
$$

such that $\left(g^{j}\right)^{\tau^{r}}=g^{j p^{r}}=g^{j}$, hence $\left(g^{j} u\right)^{\tau^{r}}=\omega^{2} g^{j} u$ and $\left(g^{-j} u^{-1}\right)^{\tau^{r}}=$ $\omega g^{-j} u^{-1}$. But there are at most $3\left(p^{r}-1\right)$ semisimple classes fixed modulo $Z(E)$, as $s_{r}\left(N_{E}(P) / Z(E)\right)=p^{r}-1$. Assertion (b) follows.

There are $p^{r / 2}+1 p$-singular classes of $E$ which meet $\mathrm{SU}_{3}\left(p^{r}\right) u$, see Step 2 of the proof of Proposition 4.4. There are $3\left(p^{r / 2}+2\right) \quad p$-singular classes of 
$E$ fixed modulo $Z(E)$ by $\tau^{r}$, and $\tau^{r}$ fixes $p^{r / 2}+4 p$-singular classes of $E$, all contained in $H$, by Proposition 4.4. Assertion (c) follows.

\section{Central eXtensions involving Simple Chevalley groups}

In this section it is shown that a counterexample to Proposition 3.1 does not contain a simple composition factor $G$ isomorphic to $\operatorname{PSL}_{2}(q)$ or $\operatorname{PSU}_{3}\left(q^{2}\right)$.

Proposition 5.1. Let $X$ be an almost simple group with a T.I. Sylow p-subgroup $P$. Let $H=O^{p^{\prime}}(X)$ and $G=H / Z(H)$. Suppose that either $G \cong \operatorname{PSL}_{2}(q)$ with $|P|=q=p^{n}$ or $G \cong \mathrm{PSU}_{3}\left(q^{2}\right)$ with $|P|=q^{3}=p^{3 n}$. If $B$ denotes a $p$-block of $X$ with defect group $\delta(B)=P$ and Brauer correspondent $b$ in $N=N_{X}(P)$, then the following assertions hold:

(a) $k(B)=k(b) \leq|P|$;

(b) $l(B)=l(b)$;

(c) $k_{0}(B)=k_{0}(b) \leq\left|P / P^{\prime}\right|$;

(d) $l(B) \leq k_{0}(B)$;

(e) If $G \cong \operatorname{PSL}_{2}(q)$, then $P$ is abelian and $k_{0}(B)=k(B)$.

Proof. Since $H$ is quasi-simple, the following holds: if $G \cong \operatorname{PSL}_{2}(q)$ then either $H \cong G$ or $H \cong \mathrm{SL}_{2}(q)$, and if $G \cong \operatorname{PSU}_{3}\left(q^{2}\right)$ then either $H \cong G$ or $H \cong \mathrm{SU}_{3}\left(q^{2}\right)$. Let $m=2$ if $G \cong \operatorname{PSL}_{2}(q)$, and $m=3$ if $G \cong \operatorname{PSU}_{3}\left(q^{2}\right)$, so that $|Z(H)| \mid m$.

If $\bar{P}$ denotes the image of $P$ in $X / Z(H)$, then $X / Z(H) \leq \operatorname{Aut}(G)$ by Proposition 1.2. Hence, $C_{X / Z(H)}(\bar{P})=1$ by $[12$, p. 311$]$, so that $C_{X}(P)=$ $Z(H)$. Thus $X$ has at most $m$ blocks of full defect: the principal block $B_{0}$, and, if $|Z(H)|=m$, faithful blocks $B_{i}$ for $i=1, m-1$. Each $B_{i}$ then covers a unique block $B_{i}^{\prime}$ of $H$. Also, the Brauer correspondents $b_{i}$ from $N$ cover, respectively, the Brauer correspondents $b_{i}^{\prime}$ from $N_{H}(P)$ of the $B_{i}^{\prime}$.

If $g \in X, T$ is a section of $X$ stable under conjugation by $g$ and $\tilde{B}$ is a block of $T$ stable under conjugation by $g$, let $f(T, g)$, resp. $f(\tilde{B}, g)$, denote the number of irreducible Brauer characters of $T$, resp. of $\tilde{B}$, which are fixed by $g$. By [11, Lemma 3.6, p. 146] and Brauer's permutation lemma, $f(T, g)$ is the number of $p$-regular conjugacy classes of $T$ fixed by $g$.

Suppose first that $X / H$ is cyclic. Then $X / H$ is generated by some $y \in X$ such that $y$ induces an outer automorphism $\gamma$ of $H$ by conjugation. By the Atlas $\left[8\right.$, p. xvi], we may assume that $\gamma$ equals either $\tau^{r}$ or $\delta \tau^{r}$, where $\delta$ is a diagonal automorphism of $H$ of order $m$ modulo the group $\operatorname{Inn}(H)$ of inner automorphisms of $H$, and $\tau^{r}$ is the field automorphism of $H$ defined by $c \rightarrow c^{p^{r}}$, for all $c \in G F\left(q^{m-1}\right)$, and some $r \mid(m-1) n$. When $X=H$, then of course $\gamma=\tau^{(m-1) n}$. By Proposition 1.2 we may assume that $y$ normalizes $P$.

Now any power of $\gamma$ must generate a subgroup of $\operatorname{Aut}(H)$ which, modulo $\operatorname{Inn}(H)$, has a generator of the form $\tau^{r^{\prime}}$ or $\delta^{ \pm 1} \tau^{r^{\prime}}$ for some $r^{\prime} \mid(m-1) n$. Since 
$\delta$ fixes every $p$-regular class of $H$, Propositions 4.3 and 4.4 imply that for all positive integers $j$,

$$
f\left(H, y^{j}\right)=f\left(N_{1}, y^{j}\right)+1, \quad f\left(H / Z(H), y^{j}\right)=f\left(N_{1} / Z(H), y^{j}\right)+1,
$$

where $N_{1}=N_{H}(P)$. Since $H$ has a unique $p$-block of defect zero, and this block has $Z(H)$ in its kernel, by $[9$, p. 228; 21, p. 488], we have

$$
f\left(B_{0}^{\prime}, y^{j}\right)=f\left(H / Z(H), y^{j}\right)-1=f\left(N_{1} / Z(H), y^{j}\right)=f\left(b_{0}^{\prime}, y^{j}\right)
$$

for all $j$. Also,

so that

$$
\sum_{i=0}^{m-1} f\left(B_{i}^{\prime}, y^{j}\right)=f\left(H, y^{j}\right)-1=f\left(N_{1}, y^{j}\right)=\sum_{i=0}^{m-1} f\left(b_{i}^{\prime}, y^{j}\right)
$$

$$
\sum_{i=1}^{m-1} f\left(B_{i}^{\prime}, y^{j}\right)=\sum_{i=1}^{m-1} f\left(b_{i}^{\prime}, y^{j}\right)
$$

if these blocks really appear in $H$. So Proposition 2.3, applied to $X / H$ acting on $B_{0}^{\prime}, b_{0}^{\prime}, \bigcup_{i=1}^{m-1} B_{i}^{\prime}$ and $\bigcup_{i=1}^{m-1} b_{i}^{\prime}$ implies that $l\left(B_{0}\right)=l\left(b_{0}\right)$ and $\sum_{i=1}^{m-1} l\left(B_{i}\right)=$ $\sum_{i=1}^{m-1} l\left(b_{i}\right)$. If $m=2$, we have $l\left(B_{1}\right)=l\left(b_{1}\right)$. If $m=3$ then $B_{1}$ and $B_{2}$ are complex conjugate blocks, as are $b_{1}$ and $b_{2}$. So $l\left(B_{1}\right)=l\left(B_{2}\right)$ and $l\left(b_{1}\right)=l\left(b_{2}\right)$ implies that $l\left(B_{i}\right)=l\left(b_{i}\right)$ for $i=1,2$.

Now suppose that $X / H$ is noncyclic. If $m=2$, let $x=\left(\nu_{\nu}^{-1}{ }_{\nu}\right)$, where $\nu$ is an element of order $2(q-1)$ in $G F\left(q^{2}\right)$; if $m=3$, let

$$
x=\left(\begin{array}{lll}
\pi^{-1} & & \\
& \pi^{-1} & \\
& & \pi^{2}
\end{array}\right),
$$

where $\pi$ has order $3(q+1)$ in $G F\left(q^{6}\right)$ (as in $\left.\S 4\right)$. Let $E$ be the group $H\langle x\rangle$ defined in $\S 4$, and let $\bar{x}$ be the image of $x$ in $E / Z(E)$. By Lemma 3.7, $X / H$ is generated by elements $y, t$ such that $y^{m} \in H, y$ induces by conjugation the same automorphism of $H$ as does $x$. Conjugation of $H$ by $t$ induces the field automorphism $\tau^{r}$ on $H$, where $r \mid(m-1) n$, and $y^{t}=y^{p^{r}} z$ for some $z \in Z(H)$ with $z^{m}=1$. Thus $H\langle y\rangle$ is normal in $X$. Now $y^{m}$ and $x^{m}$ both induce the same inner automorphism of $H$. Hence $y^{m}=\bar{x}^{m}$ if $Z(H)=1$, and $y^{m}=x^{m} z_{1}$, for some $z_{1} \in Z(H)$ if $Z(H) \neq 1$. By the proof of Lemma 3.7, $X$ may be replaced by another almost simple group isoclinic to $X$, in which we may assume $y^{m}=x^{m}$. So if $Z(H)>1$, we may assume that $H\langle y\rangle \cong E$ with $y$ corresponding to $x$, and if $Z(H)=1, H\langle y\rangle \cong E / Z(E)$ with $y$ corresponding to $\bar{x}$. So we may identify $H\langle y\rangle$ with $E$ or $E / Z(E)$, and speak of the field automorphism $\tau^{r}$ acting on $H\langle y\rangle$ with $y^{\tau^{r}}=y^{p^{r}}$. (Of course, $\tau^{r}$ and $t$ need not have the same action on $y$.)

Each $B_{i}$ covers a unique block $B_{i}^{\prime \prime}$ of $H\langle y\rangle$, which in turn covers $B_{i}^{\prime}$. Note that $H\langle y\rangle$ has $m$ blocks of defect zero, all with $Z(H)$ in their kernel, as $y$ fixes the unique defect zero block of $H$. 
Suppose that $Z(H)=1 . H\langle y\rangle$ is now identified with $E / Z(E)$, on which $t$ acts as $\tau^{r}$. If $d z_{r}(E / Z(E))$ denotes the numbers of $p$-blocks of $E / Z(E)$ of defect zero which are fixed by $\tau^{r}$, then Propositions 4.3 and 4.4, applied to $s_{r}(E / Z(E)), s_{r}\left(N_{E}(P) / Z(E)\right)$ and $d z_{r}(E / Z(E))$, yield that

$$
f(H\langle y\rangle, t)=f\left(N_{H\langle y\rangle}(P), t\right)+d z_{r}(E / Z(E)) .
$$

Hence, $f\left(B_{0}^{\prime \prime}, t\right)=f\left(b_{0}^{\prime \prime}, t\right)$. Applying this result to the subgroup $H\left\langle y, t^{j}\right\rangle$ for each positive integer $j$ yields $f\left(B_{0}^{\prime \prime}, t^{j}\right)=f\left(b_{0}^{\prime \prime}, t^{j}\right)$ for all $j$. Proposition 2.3 for the group $X / H\langle y\rangle$ acting on $B_{0}^{\prime \prime}$ and $b_{0}^{\prime \prime}$ gives $l\left(B_{0}\right)=l\left(b_{0}\right)$.

Now suppose that $Z(H)>1$. We still have $l\left(B_{0}\right)=l\left(b_{0}\right)$ by the above result. If $m=2$ then $p>2$, and if $m=3$ then $p \equiv 2(\bmod 3)$ and $n$ is odd.

If $m=2$ then $y^{q-1}=-I$ in $\operatorname{SL}_{2}(q)$. If $m=3$, then $\pi^{q+1}=\omega$ implies that $y^{q^{2}-1}=x^{q^{2}-1}=\left(\omega^{-1} I\right)^{q-1}=\omega^{-1} I$. So if we define $\omega=-1$ when $m=2$ (and leave $\omega$ alone when $m=3$ ), then $y^{\left(q^{m-1}-1\right)}=\omega^{-1} I$ in either case.

If $m=3$, then the fact that $t$ must act trivially on $Z(H)=Z(X)$ forces $\omega I=(\omega I)^{t}=\omega^{p^{r}} I$. Hence $r$ is even, i.e. $r \nmid n$. So $z^{p^{r}}=z$ whether $m=2$ or 3 .

Now for either value of $m, t^{(m-1) n / r} \in C_{X}(H)=Z(X)$, hence

$$
\begin{aligned}
y & =y^{t^{(m-1) n / r}}=y^{p^{p^{(m-1) n / r}}} z^{(m-1) n / r} \\
& =y^{q^{m-1}} z^{(m-1) n / r}=\left(\omega^{-1} I\right) y z^{(m-1) n / r} .
\end{aligned}
$$

So $z^{(m-1) n / r}=\omega I$. In particular, $m=|Z(H)| \dagger(m-1) n / r$. Thus $H\langle y\rangle=$ $H \cup\left(\bigcup_{i=1}^{m-1} H y^{i(m-1) n / r}\right)$.

Let $C$ be a conjugacy class of $H\langle y\rangle$. If $C \subseteq H$ then $C^{t}=C$ if and only if $C^{\tau^{\prime}}=C$. Suppose that $C \subseteq H y^{(m-1) n / r}$. Let $h y^{(m-1) n / r} \in C$ for some $h \in H$. Then

$$
\begin{aligned}
\left(h y^{(m-1) n / r}\right)^{t} & =h^{t}\left(y^{t}\right)^{(m-1) n / r}=h^{\tau^{r}}\left(y^{p^{r}} z\right)^{(m-1) n / r} \\
& =\left(h y^{(m-1) n / r}\right)^{\tau^{r}} z^{(m-1) n / r}=\left(h y^{(m-1) n / r}\right)^{\tau^{r}}(\omega I) .
\end{aligned}
$$

So if $C \subseteq H y^{(m-1) n / r}$ then $C^{t}=\omega C^{\tau^{r}}$. It follows that $C^{t}=C$ for $C \subseteq$ $H y^{(m-1) n / r}$ if and only if $C^{\tau^{r}}=\omega^{-1} C$. Similarly, if $C$ is a conjugacy class of $N_{H\langle y\rangle}(P)$ with $C \subseteq N_{H}(P) y^{(m-1) n / r}$ then $C^{t}=C$ if and only if $C^{\tau^{\tau}}=\omega^{-1} C$.

Let $m=2$. By Proposition 4.3, $n / r$ odd implies that $\tau^{r}$ fixes $\left(p^{r}+1\right) / 2 p$ regular conjugacy classes of $(H\langle y\rangle / Z(H))-(H / Z(H))$, but only one $p$-regular class of $H\langle y\rangle-H$ (the unique class of $H\langle y\rangle-H$ whose eigenvalues are fourth roots of unity). Thus $C^{\tau^{r}}=-C$ for exactly $p^{r}$ classes of $H\langle y\rangle-H$. Hence $C^{t}=C$ for exactly $2 p^{r}$ semisimple classes of $H\langle y\rangle$.

Similarly, Proposition 4.3 implies that $\tau^{r}$ fixes $\left(p^{r}-1\right) / 2$ semisimple classes of

$$
\left(N_{H}(P)\langle y\rangle / Z(H)\right)-\left(N_{H}(P) / Z(H)\right),
$$


but no semisimple classes of $N_{H}(P)\langle y\rangle-N_{H}(P)$. Thus $C^{\tau^{r}}=-C$ for exactly $p^{r}-1$ semisimple classes of $N_{H}(P)\langle y\rangle-N_{H}(P)$. So $C^{t}=C$ for exactly $2 p^{r}-2$ semisimple classes of $N_{H}(P)\langle y\rangle=N_{H\langle y\rangle}(P)$.

Let $m=3$. Proposition 4.6 shows that there are exactly $p^{r}$ semisimple classes $C \subseteq H y^{(m-1) n / r}$ with $C^{\tau^{r}}=\omega^{-1} C$ and $p^{r}-1$ semisimple classes $C \subseteq N_{H}(P) y^{(m-1) n / r}$ with $C^{\tau^{r}}=\omega^{-1} C$. Note that $C$ and $C^{-1}$ are in distinct cosets of $H$ for $C \subseteq H\langle y\rangle-H$, and $C^{t}=C$ if and only if $C^{-1 t}=C^{-1}$. Since $\tau^{r}$ fixes $p^{r}$ semisimple classes of $H\langle y\rangle$ contained in $H$, and $p^{r}-1$ semisimple classes of $N_{H\langle y\rangle}(P)$ contained in $N_{H}(P)$, by Proposition 4.4, it follows that $t$ fixes exactly $3 p^{r}$ semisimple classes of $H\langle y\rangle$ and $3 p^{r}-3$ semisimple classes of $N_{H\langle y\rangle}(P)$.

For both values of $m$, we have established that

$$
f(H\langle y\rangle, t)=f\left(N_{H\langle y\rangle}(P), t\right)+m .
$$

Propositions 4.3 and 4.4 imply that $m$ is the number of $p$-blocks of $E$ (in fact, of $E / Z(E))$ of defect zero which are fixed by $\tau^{r}$, and hence by $t$. So we have $\sum_{i=0}^{m-1} f\left(B_{i}^{\prime \prime}, t\right)=\sum_{i=0}^{m-1} f\left(b_{i}^{\prime \prime}, t\right)$.

Any $t^{j}$ generates a subgroup whose action on $H$ is generated by some $\tau^{r_{j}}$, where $r\left|r_{j}\right|(m-1) n$, so that $m \nmid(m-1) n / r_{j}$ and $r_{j} \nmid n$ if $m=3$. Our result in the above paragraph applied to the group $H\left\langle y, t^{j}\right\rangle$ yields that, for all positive integers $j$,

$$
\sum_{i=0}^{m-1} f\left(B_{i}^{\prime \prime}, t^{j}\right)=\sum_{i=0}^{m-1} f\left(b_{i}^{\prime \prime}, t^{j}\right) .
$$

Since $f\left(B_{0}^{\prime \prime}, t^{j}\right)=f\left(b_{0}^{\prime \prime}, t^{j}\right)$, as shown above, we have

$$
\sum_{i=1}^{m-1} f\left(B_{i}^{\prime \prime}, t^{j}\right)=\sum_{i=1}^{m-1} f\left(b_{i}^{\prime \prime}, t^{j}\right) .
$$

Now Proposition 2.3, applied to $X / H\langle y\rangle$ acting on $\bigcup_{i=1}^{m-1} B_{i}^{\prime}$ and $\bigcup_{i=1}^{m-1} b_{i}^{\prime}$ implies that $\sum_{i=1}^{m-1} l\left(B_{i}\right)=\sum_{i=1}^{m-1} l\left(b_{i}\right)$. If $m=2$ then $l\left(B_{1}\right)=l\left(b_{1}\right)$. If $m=3$ then the complex conjugacy of $B_{1}$ and $B_{2}$, as well as of $b_{1}$ and $b_{2}$, yields $l\left(B_{i}\right)=l\left(b_{i}\right)$ for $i=1,2$.

Part (b) of Proposition 5.1 is now proved. Then $k(B)=k(b)$ by Lemma 2.1.

We will now assume that $m=3$ and prove that $k_{0}(B)=k_{0}(b):$ If $g \in X$, $T$ is a $g$-stable section of $X$ and $\tilde{B}$ is a block of $T$, define $h z(T, g)$, resp. $h z(\tilde{B}, g)$, to be the number of height zero characters of $T$, resp. of $\tilde{B}$, which are fixed by $g$.

Suppose that $X / H$ is cyclic. Then $X / H$ is generated by some $y \in X$ such that $y$ induces the automorphism either $\tau^{r}$ or $\delta \tau^{r}$ of $H$ by conjugation, as 
before. Propositions 4.4 and 4.5 give

$$
\begin{aligned}
h z(H, y) & =h z\left(N_{H}(P), y\right)+1, \\
h z(H / Z(H), y) & =h z\left(N_{H}(P) / Z(H), y\right)+1 .
\end{aligned}
$$

So an argument identical to that used for Brauer characters yields $k_{0}\left(B_{i}\right)=$ $k_{0}\left(b_{i}\right)$ for $i=0,1,2$.

Suppose that $X / H$ is noncyclic, $X=H\langle y, t\rangle$ as before. If $Z(H)=1$, then $H\langle y\rangle$ is identified with $E / Z(E)$, as above, and $t$ acts as $\tau^{r}$ on $H\langle y\rangle$. Proposition 4.4 gives

$$
h z(H\langle y\rangle, t)=h z\left(N_{H\langle y\rangle}(P), t\right)+d z_{r}(E / Z(E)) .
$$

So arguing as for Brauer characters, we obtain $k_{0}\left(B_{0}\right)=k_{0}\left(b_{0}\right)$.

We may now assume that $Z(H)>1$ and $k_{0}\left(B_{0}\right)=k_{0}\left(b_{0}\right)$. Previous argument showed that $t$ fixes $3\left(p^{r}-1\right)$ semisimple classes of $N_{H\langle y\rangle}(P)$. But these have representations $(g x)^{j}$, where

$$
g=\left[\begin{array}{lll}
\sigma & & \\
& \sigma^{-q} & \\
& & \rho
\end{array}\right]
$$

as in Step 7 of the proof of Proposition 4.4, $N_{H\langle y\rangle}(P) / P \cong\langle g x\rangle$, and $t$ stabilizes $\langle g x\rangle$ as $z \in\langle g x\rangle$. Thus $t$ fixes exactly $3\left(p^{r}-1\right)$ of the linear characters of $N_{H\langle y\rangle}(P) / P$, i.e. the height zero characters of $N_{H\langle y\rangle}(P)$ which have $P$ in their kernel. There are three other characters of $N_{H\langle y\rangle}(P) / P^{\prime}$, which lie in three different blocks of $N_{H\langle y\rangle}(P)$, and hence are fixed by $t$. So $t$ fixes exactly $3 p^{r}$ height zero characters of $N_{H\langle y\rangle}(P)$.

Proposition 4.4 implies that $\tau^{r}$ fixes $p^{r / 2}+2 p$-singular classes of $E / Z(E)$. Hence it fixes $3\left(p^{r / 2}+2\right) p$-singular classes of $E$ up to multiplication by $Z(E)$, as can be seen from the list of classes in Step 2 of the proof of Proposition 4.4. By Proposition 4.6, there are $p^{r / 2}+1 p$-singular classes $C \subseteq H y^{2 n / r}$ with $C^{\tau^{r}}=\omega^{2} C$. There are $p^{r / 2}+4 p$-singular classes of $E$ which are contained in $H$, and which are fixed by $\tau^{r}$ (see Proposition 4.4). It follows that $t$ fixes exactly $3 p^{r / 2}+6 p$-singular classes of $E$.

The characters of $E$ of raised height are $3 q$ characters $\chi^{(u)}, 1 \leq u \leq 3 q+3$, with $u \neq \equiv 0(\bmod q+1)$, and three characters $\zeta^{(i)}, 0 \leq i \leq 2$, as described in Step 16 of Proposition 4.4. The characters $\chi^{(u)}$ correspond to linear characters $\nu^{u}$ of $\langle y\rangle$ (recall $y$ is identified with $x$ ), where $\nu^{u}\left(x^{-j}\right)=\mu^{u j}$ for $\mu$ a fixed complex $(3(q+1))$ th root of unity.

As $r \nmid n$, and all of the $\zeta^{(i)}$ have $Z(E)$ in their kernel, the proof of Step 16 shows that $t$ fixes all three of the $\zeta^{(i)}$.

Since $t$ stabilizes $\langle y\rangle$, it is clear that $t$ fixes $\chi^{(u)}$ if and only if $t$ fixes $\nu^{u}$. Note that $z^{2 n / r}=\omega I$ and $3 \nmid 2 n / r$ imply $z=\omega^{2 n / r} I$. Thus $y^{t}=y^{p^{r}}\left(\omega^{2 n / r} I\right)$. 
So

$$
\begin{aligned}
\chi^{(u) t} & =\chi^{(u)} \Leftrightarrow \nu^{u t^{-1}}=\nu^{u} \Leftrightarrow \nu^{u}\left(y^{p^{r}} \omega^{2 n / r} I\right)=\nu^{u}(y) \\
& \Leftrightarrow \mu^{-u p^{r}} \mu^{(2 n / r) u(q+1)}=\mu^{-u} \\
& \Leftrightarrow\left(p^{r}-1\right) u \equiv 2 n r^{-1} u(q+1)(\bmod 3(q+1)) \\
& \Leftrightarrow\left(p^{r / 2}-1\right) u \equiv 2 n r^{-1} u(q+1) /\left(p^{r / 2}+1\right)\left(\bmod 3(q+1) /\left(p^{r / 2}+1\right)\right) .
\end{aligned}
$$

Now $\left(p^{r / 2}-1, q+1\right)=(2, p-1)$ and $(q+1) /\left(p^{r / 2}+1\right)=1-p^{r / 2}+\cdots+$ $p^{\left(2 n r^{-1}-1\right) r / 2}$ is odd and congruent to $2 n / r(\bmod 3)($ as $r / 2$ is odd here). So the congruence above is equivalent to $u \equiv 0\left(\bmod (q+1) /\left(p^{r / 2}+1\right)\right)$. There are $3 p^{r / 2}$ solutions $u$ with $u \neq \equiv(\bmod q+1)$, so $3 p^{r / 2}$ of the $\chi^{(u)}$ are fixed by $t$. Hence $t$ fixes $3 p^{r / 2}+3$ characters of $E$ of positive height. It was shown above that $t$ fixes $3 p^{r}$ semisimple classes and $3 p^{r / 2}+6 p$-singular classes of $E$. Thus $t$ fixes $3 p^{r}+3 p^{r / 2}+6$ irreducible characters of $E$. Hence,

$$
h z(H\langle y\rangle, t)=3 p^{r}+3=h z\left(N_{H\langle y\rangle}(P), t\right)+d z_{r}(E) .
$$

So the now familiar argument yields $k_{0}\left(B_{i}\right)=k_{0}\left(b_{i}\right)$ for $0 \leq i \leq 2$.

The inequalities $k_{0}(b) \leq\left|P / P^{\prime}\right|, k(b) \leq|P|$ follow from Theorem 7.4 of Knörr [15], because $N_{X}(P) / P$ is supersolvable. Since every irreducible Brauer character in $b$ is an ordinary character of $N / P$ and thus lifts to an ordinary character in $b$ with $P$ in its kernel, it follows that $l(b) \leq k_{0}(b)$. This completes the proof.

\section{EXTENSIONS OF SIMPLE EXCEPTIONAL GROUPS OF LIE TYPE}

In this section we determine the block invariants of the $p$-blocks $B$ of the relevant automorphism groups $E$ of the simple exceptional groups of Lie type $G$ having a noncyclic T.I. Sylow $p$-subgroup. If $p=5$, then $G={ }^{2} F_{4}(2)^{\prime}$. If $p=3$, then $G={ }^{2} G_{2}\left(3^{2 m+1}\right), m=1,2, \ldots$ Furthermore, $G={ }^{2} B_{2}\left(2^{2 m+1}\right)$ for $p=2$.

Proposition 6.1. (a) The simple group $G$ of Tits ${ }^{2} F_{4}(2)^{\prime}$ has trivial Schur multiplier $M=1$. Its outer automorphism group $A$ is cyclic of order 2 .

(b) Let $E$ be the simple group $G$ or its automorphism group $G \cdot 2={ }^{2} F_{4}(2)$. Let $P$ be a Sylow 5-subgroup of $E$, and $N=N_{E}(P)$. Then all nonprincipal 5-blocks of $E$ have defect zero.

(c) The number $z(E)$ of 5-blocks of $E$ with defect zero and the numbers of irreducible characters of the principal blocks $B$ and $b$ of $E$ and $N$, respectively, are given in Table 4.

In particular, $k(E)=k(N)+z(E)$, and $l(E)=l(N)+z(E)$.

Proof. Assertion (a) follows from the Atlas [8, pp. xvi and 74].

(b) Considering the centralizers of 5-elements of $E$ in the character tables [8, p. 75], it follows immediately that only the principal 5-block $B$ of $E$ has positive defect. 
TABLE 4

\begin{tabular}{l|c|c|l|l|l|l|l|l}
$G$ & $E$ & $z(E)$ & $k(B)$ & $k(b)$ & $k_{0}(B)$ & $k_{0}(b)$ & $l(B)$ & $l(b)$ \\
\hline${ }^{2} F_{4}(2)^{\prime}$ & ${ }^{2} F_{4}(2)^{\prime}$ & 6 & 16 & 16 & 16 & 16 & 14 & 14 \\
& $G \cdot 2$ & 9 & 20 & 20 & 20 & 20 & 16 & 16 \\
\hline
\end{tabular}

(c) The numbers $z(E)$ of blocks with defect zero can easily be read from the character tables of $[8$, p. 75$]$.

Let $G={ }^{2} F_{4}(2)^{\prime}$. By Griess and Lyons [13] $P \simeq \mathrm{Z}_{5} \times \mathrm{Z}_{5}$, and $N_{G}(P) / P \simeq$ $\mathrm{Z}_{4} * \mathrm{SL}_{2}(3)$, where $S=\mathrm{SL}_{2}(3)$ acts transitively on $P-\{1\}$, and $\left|\mathrm{Z}_{4} * S\right|=48$. Now $N=N_{G}(P)=P \cdot U, P \cap U=1, S \leq U \cong N / P . S$ has three irreducible characters with the following multiplicities and degrees (given last): $3 \times 1$, $1 \times 3,3 \times 2$. Thus $U$ has the following multiplicities of character degrees: $6 \times 1,2 \times 3,6 \times 2$. These are the characters of $N$ which have $P$ in their kernels. In particular, $l(b)=l(N)=14$.

As $S$ acts transitively on the nontrivial characters $\lambda$ of $P, \chi=\lambda^{P S}$ is irreducible of degree 24 , for each such $\lambda$. Now $\lambda_{\mid P}^{N}$ has only 24 distinct irreducible constituents, and so $\left(\lambda^{N}, \lambda^{N}\right)_{N}=\left(\lambda, \lambda_{\mid P}^{N}\right)_{P}=2$. Therefore, $\chi$ has two extensions to $N$. Hence $k(N)=k_{0}(N)=16=k(b)=k_{0}(b)$. By the Atlas [8, p. 74], $k(B)=16=k_{0}(B)$ and $l(B)=20-z(G)=14$.

By Griess and Lyons [13], $E=G \cdot 2={ }^{2} F_{4}(2)$, and $N=N_{E}(P)=P$. $W, P \cap W=1$, where $W \simeq \mathrm{Z}_{4} * \mathrm{GL}_{2}(3)$. Now $\mathrm{GL}_{2}(3)$ has the following multiplicities of character degrees: $2 \times 1,3 \times 2,1 \times 4,2 \times 3$. Hence the numbers for $W \cong N_{E}(P) / P$ are $4 \times 1,6 \times 2,2 \times 4,4 \times 3$. In particular, $l\left(N_{E}(P)\right)=l(b)=16$. Let $\lambda$ and $\chi$ be as above. Let $T=T_{W}(\lambda)$ be the inertial subgroup of $\lambda$ in $W$. Then $|T|=4$, and $\lambda$ has four distinct extensions to $P T$. Hence $\chi$ has four distinct extensions to $N_{E}(P)$, each of degree 24 . Thus $k(b)=20=k_{0}(b)$. The Atlas [8, p. 74] implies that $k(B)=20=k_{0}(B)$ and $l(B)=16$. This completes the proof.

Proposition 6.2. (a) The simple Ree groups $G={ }^{2} G_{2}(q), q=3^{2 m+1}, m=$ $1,2, \ldots$, all have Schur multiplier $M=1$ and a cyclic outer automorphism group $A$ generated by the field automorphism $\tau$ of $\mathrm{GF}(q)$ with order $2 m+1$.

(b) $G$ has the principal 3-block $B$ and one block of defect zero consisting of the Steinberg character. Let $b$ be the Brauer correspondent of $B$ in $N_{G}(P)$, where $P$ is the Sylow 3-subgroup of $G$. Then

$$
\begin{gathered}
k(B)=q+7=k(b) \leq|P|=q^{3}, \\
k_{0}(B)=q=k_{0}(b)=\left|P / P^{\prime}\right|, \\
l(B)=q-1=l(b) .
\end{gathered}
$$


(c) Let $E=G\left\langle\tau^{r}\right\rangle \leq$ Aut $G$, where $3 \nmid|E: G|$. Then $B_{0}=B^{E}$ and $b_{0}=b^{N}$ are the principal blocks of $E$ and $N=N_{E}(P)$, respectively. Furthermore,

$$
\begin{gathered}
k\left(B_{0}\right)=k\left(b_{0}\right) \leq|P|, \\
k_{0}\left(B_{0}\right)=k_{0}\left(b_{0}\right) \leq\left|P / P^{\prime}\right|, \\
l\left(B_{0}\right)=l\left(b_{0}\right) \leq k_{0}\left(b_{0}\right) .
\end{gathered}
$$

In particular, $k(E)=k(N)+z(E)$, and $l(E)=l(N)+z(E)$, where $z(E)=$ $|E: G|$ is the number of blocks of $E$ with defect zero.

Proof. (a) follows from the Atlas [8, p. xvi].

(b) The Steinberg character of degree $q^{3}$ is by Ward's character table [25, pp. 87-88] the only projective irreducible character of $G$. By Ward's Theorem [25, p. 63] $O_{3^{\prime}}\left(N_{G}(P)\right)=1$. Therefore, all other irreducible characters of $G$ belong to the principal block $B$ of $G$ by Theorem 1.5 of Feit [11, p. 416], and Brauer's third main theorem on blocks. As $k(G)=q+8$ we have $k(B)=q+7$. From Ward's character table [25, p. 87] follows that $k_{0}(B)=q$. As $G$ has $q$ 3-regular conjugacy classes, $l(B)=q-1$.

Another application of Ward's Theorem [25, p. 63] yields that $N_{1}=N_{G}(P)=$ $P W$, where $W=\langle w\rangle$ is a cyclic group of order $|W|=q-1$ operating fixed point freely on $P / P^{\prime}$ and $\left|P / P^{\prime}\right|=q$. As $N_{1}$ has only one block $b$ it follows that $b$ has $q-1$ linear characters and one irreducible character of degree $q-1$. All other irreducible characters of $b$ have positive height. Thus $k_{0}(b)=$ $k\left[\left(P / P^{\prime}\right) W\right]=(q-1)+1=q$, and $l(b)=q-1=l(B)$. Hence Lemma 2.1 asserts that $k(b)=k(B)=q+7$.

(c) From the Atlas [8, p. xvi] follows that each outer automorphism of $G$ is a power $\tau^{r}$ of the Frobenius automorphism $\tau: x \rightarrow x^{3}$, and $|\operatorname{Out}(G)|=2 m+1$. Let $E=G\left\langle\tau^{r}\right\rangle$ for some $r \mid 2 m+1$, where $3 \nmid|E: G|=(2 m+1) r^{-1}$. Let $N=N_{E}(P)$. By [12, p. 167] $C_{E}(P) \leq P$. So $B_{0}=B^{E}$ and $b_{0}=b^{N}$ are the only blocks of $E$ and $N$ with positive defect, respectively. By Lemma 4.2 $\tau^{r}$ fixes $3^{r}$ semisimple classes of $G$. Hence it fixes $3^{r}-1$ irreducible Brauer characters of $B$. The 3-regular classes of $N_{1}=P W$ are represented by the powers of $w \in W$. As $w^{\tau^{r}}=w^{3^{r}}$, it follows that $\tau^{r}$ fixes $3^{r}-1$ irreducible Brauer characters of $b$. Thus Proposition 2.3 asserts that $l\left(B_{0}\right)=l\left(b_{0}\right)$. Hence $k\left(B_{0}\right)=k\left(b_{0}\right)$ by Lemma 2.1 .

Since $\tau^{r}$ stabilizes $P$, hence $Z(P)$ and $P^{\prime}$, and since 2 and 3 do not divide $(2 m+1) r^{-1}=\operatorname{order}\left(\tau^{r}\right)$, it follows that $\tau^{r}$ fixes all eight 3-singular classes of $G$. So $\tau^{r}$ fixes $3^{r}+7$ irreducible characters of $B$. The seven characters of $B$ with positive height differ only on the 3-singular classes. Hence they are fixed by $\tau^{r}$. So $\tau^{r}$ fixes $3^{r}$ height zero characters of $B$. Besides $3^{r}-1$ linear characters of $N_{1} / P$ it fixes the unique character of degree $q-1$. Thus Proposition 2.3 yields $k_{0}\left(B_{0}\right)=k_{0}\left(b_{0}\right)$. Since the Steinberg character St of $G$ has $|E: G|$ inequivalent extensions, it follows that $z(E)=|E: G|$ is the number of blocks 
of $E$ with defect zero, and

$$
\begin{gathered}
k(E)=k\left(B_{0}\right)+z(E)=k\left(b_{0}\right)+z(E)=k(N)+|E: G|, \\
l(E)=l(N)+z(E)=l(N)+|E: G| .
\end{gathered}
$$

Since $P$ is normal in $N$, each modular irreducible character is liftable to an irreducible ordinary character of $N$ of height zero. Thus $k_{0}\left(b_{0}\right) \geq l\left(b_{0}\right)$.

Furthermore, $k_{0}\left(b_{0}\right)=k_{0}(N)=k\left[\left(P / P^{\prime}\right) U\right]$, where $U$ is the complement of $P$ in $N=N_{E}(P)$. By Theorem 7.4 of Knörr [15] $k\left[\left(P / P^{\prime}\right) U\right] \leq\left|P / P^{\prime}\right|$ and $k\left(b_{0}\right)=k(N) \leq|P|$.

Proposition 6.3. (a) The simple Suzuki groups $G={ }^{2} B_{2}(q), q=2^{2 m+1}$, have Schur multiplier $M=1$ for $m=2,3, \ldots$, and $M \simeq \mathbf{Z}_{2} \times \mathbf{Z}_{2}$ for $m=1$. The outer automorphism group $A$ of each Suzuki group $G={ }^{2} B_{2}(q)$ is cyclic and generated by the field automorphism of order $2 m+1$.

(b) $G$ has the principal 2-block $B$ and one block of defect zero consisting of the Steinberg character St. Let $b$ be the Brauer correspondent of $B$ in $N_{G}(P)$, where $P$ is the Sylow 2-subgroup of $G$. Then

$$
\begin{gathered}
k(B)=q+2=k(b) \leq|P|=q^{2}, \\
k_{0}(B)=q=k_{0}(b)=\left|P / P^{\prime}\right|, \\
l(B)=q-1=l(b) .
\end{gathered}
$$

(c) Let $E=G\left\langle\tau^{r}\right\rangle \leq$ Aut $G$, where $2 \nmid|E: G|$. Then $B_{0}=B^{E}$ and $b_{0}=b^{N}$ are the principal blocks of $E$ and $N=N_{E}(P)$, respectively. Furthermore,

$$
\begin{gathered}
k\left(B_{0}\right)=k\left(b_{0}\right) \leq|P|, \\
k_{0}\left(B_{0}\right)=k\left(b_{0}\right) \leq\left|P / P^{\prime}\right|, \\
l\left(B_{0}\right)=l\left(b_{0}\right) \leq k_{0}\left(b_{0}\right) .
\end{gathered}
$$

In particular, $k(E)=k(N)+z(E)$ and $l(E)=l(N)+z(E)$, where $z(E)=$ $|E: G|$ is the number of blocks of $E$ with defect zero.

Proof. (a) follows immediately from the Atlas $[8, \mathrm{p}$. xvi].

(b) The Steinberg character St of $G$ is by Suzuki's character table [23, p. 143] the only projective irreducible character of $G$. Since the Sylow 2-subgroup $P$ of $G$ is strongly self-centralizing by Suzuki [23], $O\left(N_{G}(P)\right)=1$. Therefore all other irreducible characters of $G$ belong to the principal block $B$ of $G$ by Theorem 1.5 of Feit [11, p. 416] and Brauer's third main theorem on blocks. By Suzuki's character table [23, p. 143] we know that $k(B)=q+2, k_{0}(B)=q$ and $l(B)=q-1$.

Now $N_{1}=N_{G}(P)=P W$, where $W$ is a cyclic group of order $|W|=q-1$ operating fixed point freely on $P / P^{\prime}$ of order $\left|P / P^{\prime}\right|=q\left[23\right.$, p. 137]. Thus $N_{1}$ has $q-1$ linear characters and one irreducible character $\chi$ of degree $\chi(1)=$ $q-1$, and so $k_{0}(b)=q$ and $l(b)=q-1$. By [23, p. 142], $N_{1}$ has two further 
irreducible characters with degrees $\eta_{l}(1)=\frac{r}{2}(q-1), l=1,2$, where $r=\sqrt{2 q}$. Hence $k(b)=q+2 \leq|P|=q^{2}$.

(c) This assertion is proved in the same way as (c) of Proposition 6.2. We note here only that, by Lemma 4.2 and [23], the field automorphism $\tau^{r}$ of $G$, where $r \mid 2 m+1$, fixes $2^{r}$ semisimple classes of $G, 2^{r}-1$ many 2-regular classes of $N_{G}(P)$ and all three 2-singular classes of $G$. Hence $\tau^{r}$ fixes both the characters of $G$ with positive height.

\section{EXTENSIONS OF $\mathrm{PSL}_{3}(4)$}

In this section we determine the block invariants of the 3-blocks $B$ of the almost simple groups $E$ involving $G=\mathrm{PSL}_{3}(4)$. Its outer automorphism group $\operatorname{Out}(G) \simeq \mathbf{Z}_{2} \times \mathfrak{S}_{3}$, and its Schur multiplier is $M \simeq \mathbf{Z}_{4} \times \mathbf{Z}_{4} \times \mathbf{Z}_{3}$ (see Atlas [8, p. 23]). As $E$ is almost simple, $E / E^{\prime}$ is a 2-group, and the center $Z(E)$ of $E$ is a cyclic 2-group.

Let $P$ be a Sylow 3-subgroup of $G$. Then $P$ is a Sylow 3-subgroup of the groups $E$ considered here. Let $B$ be a 3-block of $E$ with defect group $\delta(B)={ }_{E} P$, and let $b$ be the Brauer correspondent of $B$ in $N=N_{E}(P)$. The number of blocks $E$ with defect zero is denoted by $z(E)$. The numbers of faithful and unfaithful blocks $B$ of $E$ with $\delta(B)={ }_{E} P$ are denoted by $f(E)$ and $u(E)$, respectively.

By the Atlas [8, p. 23] the Schur multiplier $M$ of $G$ contains elements $4_{1}$ and $4_{2}$ of order 4 and 2 of order 2. From [8, p. 24] we know that there are involutions $2_{i}, 1 \leq i \leq 3$, in $E-2 G$ or $E-G$ such that $2_{1}$ induces the graph-field and $2_{2}$ the field automorphism on $G$. Furthermore, in $\operatorname{Out}(G) 2_{3}$ is the product of $2_{1}$ and $2_{2}$.

By the Atlas [8, p. 24] we know that $2_{1}=2 B$ centralizes $P$ and inverts $4_{1}$ and $4_{2}$. Neither $2_{2}=2 C$ nor $2_{3}=2 D$ centralizes $P$. Furthermore, $2_{2}$ inverts $4_{1}$ and centralizes $4_{2}$, whereas $2_{3}$ centralizes $4_{1}$ and inverts $4_{2}$. All involutions $2_{i}$ centralize the involutions $2,4_{1}^{2}, 4_{2}^{2}$.

The various extensions of $G$ and of the covering groups $2 G, 4{ }_{i} G$ are denoted as in the Atlas [8]. There are two nonisomorphic extensions of $2 G$ by the Klein four group, which are denoted by $2 G(2 \times 2)$ and $2 G(2 \times 2)_{2}$. With this notation we state the following.

Proposition 7.1. (a) All irreducible characters of $E$ and of $N=N_{E}(P)$ have height zero.

(b) The numbers of blocks of $E$ and $N$, and their numbers of irreducible characters are given in Table 5.

In particular, $k(E)=k(N)+z(E)$ and $l(E)=l(N)+z(E)$.

Proof. By the Atlas [8, p. 24] and by Lemma 3.7 and its proof, it suffices to study the fifteen different cases given in Table 5.

From the character table of $G=\mathrm{PSL}_{3}(4)$ follows that $G$ has four blocks of defect zero consisting of the four characters with degrees $2 \times 45$ and $2 \times 63$. The 
TABLE 5

\begin{tabular}{l|r|r|r|r|r|r|r|r|r|r|r}
\multicolumn{1}{c|}{$E$} & $z(E)$ & $f(E)$ & $k(B)$ & $k(b)$ & $l(B)$ & $l(b)$ & $u(E)$ & $k(B)$ & $k(b)$ & $l(B)$ & $l(b)$ \\
\hline$G$ & 4 & 1 & 6 & 6 & 5 & 5 & 0 & & & & \\
\hline$G 2_{1}$ & 2 & 2 & 6 & 6 & 5 & 5 & 0 & & & & \\
\hline$G 2_{2}$ & 5 & 1 & 9 & 9 & 7 & 7 & 0 & & & & \\
\hline$G 2_{3}$ & 5 & 1 & 9 & 9 & 7 & 7 & 0 & & & & \\
\hline$G(2 \times 2)$ & 4 & 2 & 9 & 9 & 7 & 7 & 0 & & & & \\
\hline $2 G$ & 6 & 1 & 6 & 6 & 5 & 5 & 1 & 6 & 6 & 5 & 5 \\
\hline $2 G 2_{1}$ & 6 & 1 & 6 & 6 & 4 & 4 & 2 & 6 & 6 & 5 & 5 \\
\hline $2 G 2_{2}$ & 9 & 1 & 9 & 9 & 7 & 7 & 1 & 9 & 9 & 7 & 7 \\
\hline $2 G 2_{3}$ & 9 & 1 & 9 & 9 & 7 & 7 & 1 & 9 & 9 & 7 & 7 \\
\hline $2 G(2 \times 2)$ & 12 & 1 & 9 & 9 & 5 & 5 & 2 & 9 & 9 & 7 & 7 \\
\hline $2 G(2 \times 2)_{2}$ & 6 & 1 & 9 & 9 & 8 & 8 & 2 & 9 & 9 & 7 & 7 \\
\hline $4_{1} G$ & 6 & 2 & 6 & 6 & 5 & 5 & 2 & 6 & 6 & 5 & 5 \\
\hline $4_{1} G 2_{3}$ & 9 & 2 & 9 & 9 & 7 & 7 & 2 & 9 & 9 & 7 & 7 \\
\hline $4_{2} G$ & 8 & 2 & 6 & 6 & 5 & 5 & 2 & 6 & 6 & 5 & 5 \\
\hline $4_{2} G 2_{2}$ & 13 & 2 & 9 & 9 & 7 & 7 & 2 & 9 & 9 & 7 & 7 \\
\hline
\end{tabular}

remaining six characters of $G$ with degrees $1,20,3 \times 35$ and 64 belong to the principal 3-block $B_{0}$. Since $G$ has nine 3-regular conjugacy classes, $l\left(B_{0}\right)=5$. Now $N=N_{G}(P)$ is a semidirect product of $P$ by a quaternion group $Q$ of order 8 acting fixed point freely on $P$. Thus $N$ has only the principal 3-block $b_{0}$, and its irreducible characters are of degrees $4 \times 1,2$ and 8 . In particular, $k\left(b_{0}\right)=6=k\left(B_{0}\right)$ and $l\left(b_{0}\right)=5=l\left(B_{0}\right)$, which proves assertion $(\mathrm{b})$ in the case $E=G$.

Using the Atlas [8] it is now easy to determine the numbers $z(E)$ of blocks of $E$ with defect zero and the degrees of their characters in the remaining cases different from $2 G(2 \times 2)$ and $2 G(2 \times 2)_{2}$.

Suppose $E=G 2_{1}$. Then $N=P Q \times 2_{1}$ by the Atlas [8, p. 23]. Therefore, $N$ has two blocks $b$ isomorphic to the group algebra $F[P Q]$. Thus $l(b)=5$ and $k(b)=6$. Let $B$ be the Brauer correspondent of $b$ in $E$. From the Atlas [8, p. 24] we know that $k(B)=6=k(b)$. Thus $l(B)=5=l(b)$ by Lemma 2.1.

In the cases $E=G 2_{i}, i=2,3, N$ has only one block $b$ because no element of $E-G$ centralizes $P$. It follows from Clifford theory that $k(b)=9$ and $l(b)=7$ in each case. Using the above arguments we obtain $k(B)=9=k(b)$ and $l(B)=7=l(b)$.

Suppose that $E=G(2 \times 2)$. By the Atlas [8, p. 24] we may assume that $E=\left(G 2_{2}\right) 2_{1}$. Let $N=N_{E}(P)$. Then $N=\left[P Q 2_{2}\right] \times\left\langle 2_{1}\right\rangle$ by [8, p. 23]. 
Therefore, $N$ has two 3-blocks $b$ which are both isomorphic to the group algebra $F\left[\mathrm{PQ2}_{2}\right]$. Hence $k(b)=9$ and $l(b)=7$ by the previous case. Let $B$ be the Brauer correspondent of $b$ in $E$. Then by [8, footnote of $\mathrm{p}$. xxii and p. 24], each of the two blocks $B$ of $E$ with $\delta(B)={ }_{E} P$ has nine irreducible characters with degrees $2 \times 1,2 \times 20,2 \times 35,70$ and $2 \times 64$. Thus $l(B)=$ $l(b)=7$ by Lemma 2.1. Furthermore, $z(E)=4$, and the character degrees in the blocks of defect zero are $2 \times 90$ and $2 \times 126$.

In the remaining cases it suffices to determine the block invariants of the faithful blocks $B$ with $\delta(B)={ }_{E} P$.

By the Atlas [8] and the previous cases all assertions hold for the three cases $E \in\left\{2 G, 4_{1} G, 4_{2} G\right\}$ because the faithful blocks of $E$ and $N$ have the same block invariants as the unfaithful principal blocks of $E$ and $N$, respectively. Moreover, all irreducible characters of all occurring central extensions' $H$ of $G$ have height zero, and so have the irreducible characters of $N_{H}(P)$. If $E$ is an arbitrary almost simple group, then $H=O^{3^{\prime}}(E)$ is such a covering group of $G$. As $E / H$ is a 2-group, every irreducible character of $E$ and also of $N_{E}(P)$ has height zero. Thus (a) holds.

Let $E=2 G 2_{1}$ and $N=N_{E}(P)$. Then the center $Z(E)=\langle z\rangle$ has order 2. We may assume that an involution 21 of $E-2 G$ induces the graph field automorphism on $G$. Hence by [8, p. 24], $C_{E}(P)=P \times\left\langle z, 2_{1}\right\rangle$ and $N=\left[P\left(\langle z\rangle \times Q_{8}\right)\right]\left\langle 2_{1}\right\rangle$. Furthermore, $2_{1}$ and $2_{1} z$ are conjugate in $N$. Thus $2_{1}^{y}=2_{1} z$ for some $y \in Q_{8}$, and $N$ has three blocks of full defect, one of which, $b$ say, is faithful on $Z(E)$. Let $B$ be its Brauer correspondent in $E$. By [8, p. 24], $E$ has 24 conjugacy classes, four of which are 3-singular. As $z(E)=6$ and $E / Z(E) \cong G \cdot 2_{1}$, it follows that $k(B)=6$ and $l(B)=4$. Let $1, g, a, c, d$ be representatives of the conjugacy classes of $Q_{8}$, where $a^{2}=c^{2}=d^{2}=g$ and $g^{2}=1$. By [8, p. 23], 2 centralizes $Q_{8}$ modulo $Z(E)$. We may assume that $a^{2_{1}}=a z, c^{2_{1}}=c z, d^{2_{1}}=d$ and $2_{1}^{a}=2_{1} z$. Therefore $N / P \cong\left[\langle z\rangle \times Q_{8}\right]\left\langle 2_{1}\right\rangle$ has 14 conjugacy classes with representatives $1, z, g, g z, a, 2_{1} a, c, 2_{1} c, d, d 2_{1}, d z, d z 2_{1}, 2_{1}$ and $2_{1} g z$. By the case $G 2$, the two unfaithful blocks of $N$ each have five modular irreducible characters. Thus $l(b)=4=l(B)$. Hence $k(b)=k(B)=6$ by Lemma 2.1.

Let $E \in\left\{2 G 2_{2}, 2 G 2_{3}\right\}$. Since no element of $E-2 G$ centralizes $P$ by the Atlas [8, p. 24], we have in each case that $O_{3^{\prime}}(N)=\langle z\rangle=Z(N)$, where $N=N_{E}(P)$. Therefore, $N$ has one faithful block $b$ and the principal block $b_{0}$. Now $N / P \cong\left[\langle z\rangle \times Q_{8}\right]\left\langle 2_{i}\right\rangle$. By $\left[8\right.$, p. 24] each $2_{i}$ fuses two 4-classes of $Q_{8}$ and fixes the third. Hence $N / P$ has 14 irreducible characters. As $l\left(b_{0}\right)=7$ by the case $G 2_{i}$, it follows that $l(b)=7$. Let $B$ be the Brauer correspondent of $b$ in $E$. By [8, p. 24] in each case $E$ has 27 conjugacy classes, four of which are 3-singular. As $z(E)=9, l(B)=7$ and $k(B)=9$ by the case $G 2_{i}$. Thus $k(b)=k(B)=9$ by Lemma 2.1 .

Let $E \in\left\{4_{1} G 2_{3}, 4_{2} G 2_{2}\right\}$. Then $2_{2}$ and $2_{3}$ centralize $4_{1}$ and $4_{2}$, respectively. But they do not centralize $P$. Let $N=N_{E}(P)$. Then $O_{3^{\prime}}(N)=\langle z\rangle=$ 
$Z(N)$ is cyclic of order 4 , and $N / P=\left[\langle z\rangle \times Q_{8}\right]\left\langle 2_{i}\right\rangle$. By [8, p. 24] each $2_{i}$ fuses two four classes of $Q_{8}$ and fixes the third. Hence $N / P$ has 28 irreducible characters. $N$ has two unfaithful blocks $b_{0}$ and $b_{1}$, and two faithful blocks $b_{2}$ and $b_{3}$ which are complex conjugate. By the previous case $l\left(b_{j}\right)=7$ for $j=0,1$. Thus $l\left(b_{j}\right)=7$ for $j=2,3$. Let $B_{j}$ be the Brauer correspondent of $b_{j}$ in $E$. By [8, p. 24] the groups $4_{1} G 2_{3}$ and $4_{2} G 2_{2}$ have 45 and 49 conjugacy classes and 9 and 13 blocks of defect zero, respectively. Furthermore, both groups each have eight 3-singular classes. Since $B_{2}$ and $B_{3}$ are complex conjugate, it follows from the cases $2 G 2_{i}$ that $l\left(B_{j}\right)=7$ and $k\left(B_{j}\right)=9$ for $j=2,3$. Hence $k\left(b_{j}\right)=k\left(B_{j}\right)=9$ by Lemma 2.1 .

Let $E / 2 G \cong\left\langle 2_{1}\right\rangle \times\left\langle 2_{2}\right\rangle$ and $Z(E)=\langle z\rangle$ of order 2, where $2_{1}$ and $2_{2}$ induce the graph field automorphism and the field automorphism on $G$, respectively. Furthermore, assume that $2_{1}$ and $2_{2}$ are involutions in $E$. Since the different actions $\left(2_{2}\right)^{2_{1}}=2_{2}$ and $\left(2_{2}\right)^{2_{1}}=2_{2} z$ give rise to two different automorphisms of $2 \mathrm{G2}_{2}$, there are two nonisomorphic almost simple groups $E_{0}=2 G(2 \times 2)$ and $E_{1}=2 G(2 \times 2)_{2}$, respectively. Furthermore, if $X$ is an almost simple group with center $Z(X)$ of order $2, G \cong \mathrm{PSL}_{3}(4)$ and $X / G \cong\left\langle 2_{1}\right\rangle \times\left\langle 2_{2}\right\rangle$, then by Lemma $3.7 X$ is isoclinic to either $E_{0}$ or $E_{1}$.

Let $E \in\left\{E_{0}, E_{1}\right\}$. As $2_{1}$ centralizes $P$ and $2_{2}$ does not, $C_{E}(P)=$ $P \times\left\langle z, 2_{1}\right\rangle$. Furthermore, by the case $2 G 2_{1}$, we know that $2_{1}$ and $2_{1} z$ are conjugate in $N=N_{E}(P)$. Therefore $N$ has three blocks, one of which, $b$ say, is faithful on $Z(E)$. Let $B$ be its Brauer correspondent in $E$.

Now $N / P=\left[\left(\langle z\rangle \times Q_{8}\right)\left\langle 2_{2}\right\rangle\right]\left\langle 2_{1}\right\rangle$. Using the Cayley system, Dr. G. Schneider (Essen University) has computed the character tables of $N$ for both cases $E=$ $E_{0}$ and $E=E_{1}$. If $E=E_{0}$, then $N / P$ has $k(N / P)=19$ classes, and if $E=E_{1}$, then $k(N / P)=22$. From the case $G(2 \times 2)$ follows that $l(b)=5$ and $l(b)=8$ for $E_{0}$ and $E_{1}$, respectively. In any case $N$ has 27 conjugacy classes, because its number of 3-singular classes is 8 and 5 for $E_{0}$ and $E_{1}$, respectively. Therefore $k(b)=9$ for each group $E \in\left\{E_{0}, E_{1}\right\}$.

Let $E=E_{j}$ for $j=0$ or 1 . We freely use the notation of [8, p. 24] for characters and classes of subgroups of $E$.

From [8, p. 24] $2 G 2_{1}$ has a unique faithful irreducible character of degree 20, which therefore has two extensions to $E$. Also $2 G 2_{2}$ has four faithful characters of degree 10 , which hence are fused in pairs by $2_{1}$. Now $2_{1}$ must fix the unique irreducible character of $2 G 2_{2}$ of degree 56 .

Let $\chi_{k}^{ \pm}$denote the two extensions of $\chi_{k} \in \operatorname{Irr}(2 G)$ to $2 G 2_{2}$ for $k=$ $15,16,17,18$. Let $\widehat{2 C}$ be a preimage of the class $2 C$ of $G 2_{2}$ in $2 G 2_{2}$. We may assume $2_{2} \in \widehat{2 C}$. Since $\chi_{k}^{ \pm}\left(2_{2}\right) \neq 0$ for $k=15,16,18$, each such $\chi_{k}^{ \pm}$is fixed by $2_{1}$ if and only if $2_{2}^{2_{1}}=2_{2}$, i.e. $E=E_{0}$.

Let $\widehat{8 B}, \widehat{8 C}$ be the preimages of the conjugacy classes $8 B, 8 C$, respectively, which are shown in the table for the faithful characters of $2 G 2_{1}$. Now $2_{2}$ fuses the classes $4 B$ and $4 C$ of $G$, by [8, p. 24], so $2_{2}$ fixes $8 A$ and fuses $8 B$ and 
$8 C$. But $2_{2}$ fixes the extensions of $\chi_{18}$ to $2 G 2_{1}$ if and only if $2_{1}$ fixes $\chi_{18}^{ \pm}$, i.e. if and only if $j=0$. So $\widehat{8 B}^{2_{2}}=\widehat{8 C} z$ if $j=0$ and $\widehat{8 B}^{2}=\widehat{8 C}$ if $j=1$.

Suppose that $E=E_{0}$, i.e. $2_{2}^{2_{1}}=2_{2}$. Then $\widehat{8 B}^{2}=\widehat{8 C} z$ yields that $2_{2}$ fuses the two extensions of $\chi_{17}$ to $2 G 2_{1}$. Then $2_{1}$ fuses $\chi_{17}^{ \pm}$. It follows that $2_{1}$ fixes seven of the thirteen faithful characters of $2 \mathrm{G2}_{2}$. So the faithful block $B$ has the following character degrees: $2 \times 20,2 \times 56,4 \times 64,1 \times 140$. Then $k(B)=9$. There are eight faithful blocks of defect zero, with character degrees $4 \times 36,4 \times 90$. Since $z(G(2 \times 2))=4$, we have $z(E)=12$.

Suppose that $E=E_{1}$, i.e. $2_{2}^{2_{1}}=2_{2} z$. Then $\widehat{8 B}^{2_{2}}=\widehat{8 C}$ implies that $2_{2}$ fixes the two extensions of $\chi_{17}$ to $2 G 2_{1}$. Then $2_{1}$ fixes $\chi_{17}^{ \pm}$. So $2_{1}$ fixes three of the thirteen faithful characters of $2 G 2_{2}: \chi_{17}^{ \pm}$and the degree 56 character. Thus $B$ has the following character degrees: $2 \times 20,2 \times 56,1 \times 128,4 \times 70$. So $k(B)=9$. There are two faithful blocks of defect zero, with character degrees $1 \times 72,1 \times 180$. Thus $z(E)=4+2=6$. As $k(B)=9=k(b)$, Lemma 2.1 asserts that $l(B)=l(b)$. Hence $l(B)=5$ and $l(B)=8$ for $E=E_{0}$ and $E=E_{1}$, respectively. This completes the proof.

\section{EXTENSIONS OF SPORADIC SIMPLE GROUPS}

In this section we determine the block invariants of the $p$-blocks $B$ of the almost simple groups $E$ involving a simple sporadic group $G$ with a T.I. Sylow p-subgroup $P$.

Let $N=N_{E}(P)$, and let $b$ be the Brauer correspondent of $B$ in $N$. The number of $p$-blocks of defect zero of $E$ is denoted by $z(E)$. Let $a(E)$ be the number of $p$-blocks $B$ of $E$ with defect groups $\delta(B)={ }_{E} P$. Again the faithful and the principal block $B_{0}$ of $E$ have the same block invariants.

Using this and the notation of the Atlas [8] we have

Proposition 8.1. The numbers of blocks of $E$ and $N$, and their numbers of irreducible characters are given in Table 6.

In particular, $k(E)=k(N)+z(E)$ and $l(E)=l(N)+z(E)$.

TABLE 6

\begin{tabular}{l|l|r|r|r|r|r|r|r|r|r}
$G$ & $E$ & $p$ & $z(E)$ & $a(E)$ & $k(B)$ & $k(b)$ & $k_{0}(B)$ & $k_{0}(b)$ & $l(B)$ & $l(b)$ \\
\hline$M_{11}$ & $M_{11}$ & 3 & 1 & 1 & 9 & 9 & 9 & 9 & 7 & 7 \\
\hline$M^{c} L$ & $M^{c} L$ & 5 & 5 & 1 & 19 & 19 & 13 & 13 & 12 & 12 \\
\hline & $G 2$ & 5 & 7 & 1 & 26 & 26 & 20 & 20 & 18 & 18 \\
\hline & $3 G$ & 5 & 9 & 3 & 19 & 19 & 13 & 13 & 12 & 12 \\
\hline$J_{4}$ & $J_{4}$ & 11 & 13 & 1 & 49 & 49 & 42 & 42 & 40 & 40 \\
\hline
\end{tabular}


Proof. By the Atlas [8, p. vii] the Schur multipliers $M$ and outer automorphism groups $A$ of the simple Mathieu group $M_{11}$ and Janko group $J_{4}$ are trivial. Furthermore, the simple McLaughlin group $M^{c} L$ has $M \simeq \mathbf{Z}_{3}$ and $A \simeq \mathbf{Z}_{2}$, and $A$ does not centralize $M$ by $[8$, p. 101].

Let $G$ be one of these simple sporadic groups and let $P$ be a Sylow $p$ subgroup of $G$, where $p$ is a prime listed. Then the character table of $E$ is given in the Atlas [8, pp. 18, 100 and 188]. For $E=G$ the character tables of $N=N_{E}(P)$ can be found in Ostermann [18]. In each of the cases $E \in\left\{M_{11}, M^{c} L, J_{4}\right\}$ all assertions follow from the character tables. So we may assume that $G=M^{c} L$ and $E \in\{3 G, G 2\}$.

Let $E=3 G$. Then $E$ and $N$ each have three blocks $B_{i}$ and $b_{i}, i=$ $0,1,2$, respectively, such that $b_{i}$ is the Brauer correspondent of $B_{i}$ in $N$. By [8, p. 101] each $B_{i}$ has the same block invariants as the principal 5-block $b_{0}$ of $N$. Since $O_{5^{\prime}}(E)=O_{5^{\prime}}(N)=\mathrm{Z}_{3}$ is the kernel of $B_{0}$ and of $b_{0}$, we know from the case $E / O_{s^{\prime}}(E) \simeq G$ that $B_{0}$ and $b_{0}$ have the same block invariants. Thus all assertions hold in this case.

Suppose that $E=G 2$. By the Atlas [8, p. 100] no element of $E$ outside $G$ centralizes $P$. Thus $O_{5^{\prime}}(N)=1$, and only the principal block $B_{0}$ of $E$ has highest defect. Let $b_{0}$ be its Brauer correspondent in $N=N_{E}(P)$. By [8, p. 100] we have $k\left(B_{0}\right)=26, k_{0}\left(B_{0}\right)=20$ and $l\left(B_{0}\right)=18$. Now $b_{0}=F N$. Using the character table $[8, \mathrm{p} .100]$ and the CAYLEY computer program, G. Schneider (Essen University) computed the character table of $N$. It follows that $N$ has 26 irreducible ordinary characters with degrees $1,2,20,24$ and 40 and multiplicities $8,10,4,2$ and 2, respectively. In particular, $k_{0}\left(B_{0}\right)=20=$ $k_{0}\left(b_{0}\right)$. Since $P$ is a T.I. Sylow 5-subgroup of $E$, Lemma 2.1 implies that $l\left(B_{0}\right)=18=l\left(b_{0}\right)$. This completes the proof.

\section{THE MAIN THEOREM}

After all these preparations we can prove the main result of this article in this section.

Lemma 9.1. All assertions of Proposition 3.1 hold.

Proof. Let $X$ be a finite group with a T.I. Sylow $p$-subgroup $P$ which is a counterexample to Proposition 3.1. Let $H=O^{p^{\prime}}(X)$. Then by Lemma 3.2 and Proposition 3.8 there is a nonabelian simple group $G$ with a noncylic Sylow $p$ subgroup $P_{1}$ and Schur multiplier $M$ such that (a) $P_{1} \simeq P$, (b) $G \simeq H / Z(H)$, (c) $Z(H)=O_{p^{\prime}}(X)=Z(X)$ is a cyclic epimorphic image of $M$, and (d) $X / H$ is isomorphic to a subgroup of the outer automorphism group $\operatorname{Out}(G)$ of $G$. The possible simple groups $G$ are listed in Proposition 1.3.

Now Proposition 5.1 asserts that $G$ is neither isomorphic to $\operatorname{PSL}_{2}(q)$ nor to $\mathrm{PSU}_{3}\left(q^{2}\right)$. The simple Suzuki groups ${ }^{2} B_{2}(q)$ and Ree groups ${ }^{2} G_{2}(q)$ are excluded by Propositions 6.3 and 6.2, respectively. From Proposition 7.1 follows 
that $G \not \mathrm{PSL}_{3}(4)$ when $p=3$. If $p=5$ then $G \not{ }^{2} F_{4}(2)^{\prime}$ by Proposition 6.1. Finally we have shown in Proposition 8.1 that $G$ is none of the sporadic simple groups $M_{11}, M^{c} L$ or $J_{4}$.

Therefore, $X$ is not a counterexample by Proposition 1.3.

We now state the main theorem of this article.

Theorem 9.2. Let $G$ be a finite group with T.I. Sylow p-subgroup P. Let $B$ be a p-block of $G$ with defect group $\delta(B)={ }_{G} P$ and Brauer correspondent $b$ in $N=N_{G}(P)$. Then

(a) $k(B)=k(b)$;

(b) $k_{0}(B)=k_{0}(b)$;

(c) $l(B)=l(b)$;

(d) $k(G)=k(N)+z(G)$, where $z(G)$ denotes the number of $p$-blocks of defect zero in $G$.

Proof. Assertions (a) and (b) hold by Lemma 9.1 and Proposition 3.1. Now (a) and (c) are equivalent by Lemma 2.1 because $P$ is a T.I. Sylow $p$-subgroup.

Theorem 8.14 of $[11$, p. 135] asserts that every $p$-block $B$ with defect group $\delta(B) \neq_{G} P$ has defect zero. By Brauer's first main theorem on blocks the groups $G$ and $N$ have the same number of blocks with defect group $P$. Thus (a) implies $k(G)=k(N)+z(G)$ because every block $b$ of $N$ has defect group $\delta(b)=P$ by Corollary 6.9 of $[11$, p. 127]. This completes the proof.

Theorem 9.2(d) confirms Alperin's conjecture stated in the introduction. Also Brauer's height zero conjecture holds for groups with T.I. Sylow $p$-subgroups.

Corollary 9.3. Let $G$ be a finite group with a T.I. Sylow p-subgroup P. Let $B$ be a p-block of $G$ with defect group $\delta(B)={ }_{G} P$.

Then every irreducible character $\chi$ of $B$ has height $\mathrm{ht}(\chi)=0$ if and only if $P$ is abelian.

Proof. Let $b$ be the Brauer correspondent of $B$ in $N=N_{G}(P)$. Then $k(B)=$ $k(b)$ and $k_{0}(B)=k_{0}(b)$ by Theorem 9.2. Thus $k(B)=k_{0}(B)$ if and only if $k(b)=k_{0}(b)$. Therefore Theorem 9 of Reynolds [20] completes the proof.

Brauer conjectured that the following result holds in any group $G$ without any condition on the Sylow $p$-subgroups $P$ of $G$. So far, it has not been proved for groups with a normal Sylow $p$-subgroup.

Corollary 9.4. Let $G$ be a finite group with a T.I. Sylow p-subgroup $P$ such that $P$ is not normal in $G$. Let $B$ be a p-block of $G$ with defect group $\delta(B)={ }_{G} P$. Then $k(B) \leq|P|$ and $k_{0}(B) \leq\left|P / P^{\prime}\right|$.

Proof. By Lemma 3.2 we may assume that $P$ is neither cyclic nor a generalized quaternion 2-group. By Lemma 3.3 and the proofs of Lemma 3.4 and Lemma 3.7 we may assume that there exists a group $X$ with a T.I. Sylow $p$-subgroup $P_{1} \cong P$ having a $p$-block $B_{1}$ with $\delta\left(B_{1}\right)={ }_{X} P_{1}$ such that $k(B)=k\left(B_{1}\right)$, $k_{0}(B)=k_{0}\left(B_{1}\right)$ and $O^{p^{\prime}}(X) / Z(X)$ is one of the simple groups listed in Propo- 
sition 1.3. Furthermore, either $X$ is almost simple or $X$ is as described in the conclusion of Lemma 3.7. In the former case, both assertions hold by Propositions 5.1, 6.1, 6.2, 6.3, 7.1 and 8.1. If $X$ is not almost simple, as in Lemma 3.7, let $b_{1}$, a $p$-block of $N_{1}=N_{X}\left(P_{1}\right)$, be the Brauer correspondent of $B_{1}$. Then $k\left(B_{1}\right)=k\left(b_{1}\right)$ and $k_{0}\left(B_{1}\right)=k_{0}\left(b_{1}\right)$ by Propositions 3.1 and 3.8. Also, Lemma 3.7 shows that $N_{1} / P_{1}$ is supersolvable. The assertions follow from Theorem 7.4 of Knörr [15].

Remark 9.5. Two finite-dimensional $F$-algebras $B$ and $b$ are called stably equivalent if their module categories $\operatorname{Mod} B$ and $\operatorname{Mod} b$ are equivalent modulo their projective objects and projective homomorphisms. It has long been conjectured that two such algebras have the same number $l(B)=l(b)$ of nonisomorphic nonprojective simple modules.

Block ideals $B$ of group algebras with a T.I. Sylow $p$-subgroup $P$ of a finite group $G$ as defect group and their Brauer correspondents $b$ in $N=N_{G}(P)$ form important examples of stably equivalent indecomposable algebras, (see [11, p. 118]). Theorem 9.2(c) supplies some further support to that conjecture which in general is still open.

Remark 9.6. All assertions of Theorem 9.2 and its Corollaries 9.3 and 9.4 can be considered as generalizations of the theory of blocks with cyclic defect groups. Also the results of [3] and [4] on minimal degrees of faithful irreducible characters in groups with a nonnormal T.I. Sylow $p$-subgroup $P$ are similar generalizations. But the deepest result on blocks $B$ with cyclic defect groups does not hold in general for groups with a T.I. Sylow subgroup. Whereas in such a $p$-block $B$ all decomposition numbers are zero or one, the principal 5-block $B_{0}$ of the sporadic McLaughlin group $M^{c} L$ has an irreducible character of degree 3520 in which the modular irreducible character of degree 21 occurs twice as a constituent. Of course the Sylow 5-subgroup $\delta\left(B_{0}\right)=P$ of $M^{c} L$ is a T.I. set. We are grateful to Dr. Hiss (Aachen) for informing us about his work on the 5-decomposition numbers of $M^{c} L$ prior to publication.

\section{ACKNOWLEDGEMENT}

The first author gratefully acknowledges financial support by the Deutsche Forschungsgemeinschaft, Bonn, W. Germany, enabling him to visit Essen University in January, May, June 1985 and December 1986. The second author visited the Northern Illinois University in DeKalb and the University of Chicago in April 1986. His visit in the States was supported by means of the Exchange Programme between the National Science Foundation of the USA and the Deutsche Forschungsgemeinschaft. He also benefitted from an "Akademie-Stipendium" of the Stiftung Volkswagenwerk. The authors thank G. Schneider and T. Ostermann for all their help with the computation of character tables, and B. Külshammer for useful discussions. They also would like to thank the referee for several helpful suggestions. 


\section{REFERENCES}

1. J. L. Alperin, Local representation theory, Proc. Sympos. Pure Math., vol. 37, Amer. Math. Soc., Providence, R.I., 1980, pp. 369-375. (MR 82a:20015)

2. M. Aschbacher, Finite group theory, Cambridge Univ. Press, Cambridge, 1986.

3. T. R. Berger, P. Landrock and G. Michler, Minimal degrees of faithful characters of finite groups with a T.I. Sylow p-subgroup, Proc. Amer. Math. Soc. 99 (1987), 15-21.

4. H. I. Blau, On linear groups with a cyclic or T.I. Sylow subgroup, J. Algebra 114 (1988), 268-285.

5. A. Borel et al., Seminar on algebraic groups and related finite groups, Lecture Notes in Math., vol. 131, Springer, Heidelberg, 1970. (MR 41 \#3484)

6. R. W. Carter, Finite groups of Lie type, Wiley, New York, 1985.

7. G. H. Cliff, On modular representations of p-solvable groups, J. Algebra 47 (1977), 129-137. (MR $56 \# 3108$ )

8. J. H. Conway, R. T. Curtis, S. P. Norton, R. A. Parker and R. A. Wilson, Atlas of finite groups, Clarendon Press, Oxford, 1985.

9. L. Dornhoff, Group representation theory, Marcel Dekker, New York, 1971. (MR 50 \#458)

10. V. Ennola, On the characters of the finite unitary groups, Ann. Acad. Sci. Fenn. Ser. A I Math. 323 (1963), 3-33. (MR 25, 3082)

11. W. Feit, The representation theory of finite groups, North Holland, Amsterdam, 1982. (MR 83g:20001)

12. D. Gorenstein and R. Lyons, On finite groups of characteristic 2-type, Mem. Amer. Math. Soc. No. 276 (1982). (MR 82h:20021)

13. R. L. Griess and R. Lyons, The automorphism group of the Tits simple group ${ }^{2} F_{4}(2)^{\prime}$, Proc. Amer. Math. Soc. 52 (1975), 75-78. (MR 52 \#10880)

14. B. Huppert, Endliche Gruppen. I, Springer, Heidelberg, 1967. (MR 84i:20001a)

15. R. Knörr, On the number of characters in a p-block of a p-solvable group, Illinois J. Math. 28 (1984), 181-210. (MR 85d:20006)

16. B. Külshammer, On p-blocks of p-solvable groups, Comm. Algebra 9 (1981), 1763-1785. (MR 83c:20017)

17. T. Okuyama and Y. Tsushima, Local properties of p-block algebras of finite groups, Osaka J. Math. 20 (1983), 33-41. (MR 84i:20012)

18. T. Ostermann, Irreduzible Charaktere von Sylownormalisatoren der sporadischen einfachen Gruppen, Dissertation, Universität Essen, 1986.

19. L. Puig, Structure locale dans les groupes finis, Mémoire, no. 47, Bull. Soc. Math. France, 1976, 132 pp. (MR 56 \#8704)

20. W. F. Reynolds, Blocks and normal subgroups of finite groups, Nagoya Math. J. 22 (1963), 15-32. (MR 27, 3690)

21. W. A. Simpson and J. S. Frame, The character-tables for $\operatorname{SL}(3, q), \operatorname{SU}\left(3, q^{2}\right), \operatorname{PSL}(3, q)$, $\operatorname{PSU}\left(3, q^{2}\right)$, Canad. J. Math. 25 (1973), 486-494. (MR 49 \#398)

22. R. Steinberg, The representations of $\mathrm{GL}(3, q), \operatorname{GL}(4, q), \operatorname{PGL}(3, q)$ and $\operatorname{PGL}(4, q)$, Canad. J. Math. 3 (1951), 225-235. (MR 13, 10)

23. M. Suzuki, On a class of doubly transitive groups, Ann. of Math. (2) 75 (1962), 105-145. (MR 25, 112)

24. _ Finite groups of even order in which Sylow 2-subgroups are independent, Ann. of Math. (2) 80 (1964), 58-77. (MR 25, 145)

25. H. N. Ward, On Ree's series of simple groups, Trans. Amer. Math. Soc. 121 (1966), 62-89. (MR 33 \#5752)

Department of Mathematical Sciences, Northern Illinois University, DeKalb, IlliNOIS 60115

Department of Mathematics, University of Essen, 4300 Essen, West Germany 